\title{
Plantar pressures in diabetic polyneuropathy : the influence of gait pattern and exercise therapy
}

Citation for published version (APA):

Melai, T. (2013). Plantar pressures in diabetic polyneuropathy : the influence of gait pattern and exercise therapy. [Doctoral Thesis, Maastricht University]. Maastricht University. https://doi.org/10.26481/dis.20131011tm

Document status and date:

Published: 01/01/2013

DOI:

10.26481/dis.20131011tm

Document Version:

Publisher's PDF, also known as Version of record

\section{Please check the document version of this publication:}

- A submitted manuscript is the version of the article upon submission and before peer-review. There can be important differences between the submitted version and the official published version of record.

People interested in the research are advised to contact the author for the final version of the publication, or visit the DOI to the publisher's website.

- The final author version and the galley proof are versions of the publication after peer review.

- The final published version features the final layout of the paper including the volume, issue and page numbers.

Link to publication

\footnotetext{
General rights rights.

- You may freely distribute the URL identifying the publication in the public portal. please follow below link for the End User Agreement:

www.umlib.nl/taverne-license

Take down policy

If you believe that this document breaches copyright please contact us at:

repository@maastrichtuniversity.nl

providing details and we will investigate your claim.
}

Copyright and moral rights for the publications made accessible in the public portal are retained by the authors and/or other copyright owners and it is a condition of accessing publications that users recognise and abide by the legal requirements associated with these

- Users may download and print one copy of any publication from the public portal for the purpose of private study or research.

- You may not further distribute the material or use it for any profit-making activity or commercial gain

If the publication is distributed under the terms of Article $25 \mathrm{fa}$ of the Dutch Copyright Act, indicated by the "Taverne" license above, 


\section{Plantar pressures in diabetic polyneuropathy}

The influence of gait pattern and exercise therapy

Tom Melai 
Cover design: Esther Theeven-van Hinsberg

(C) Copyright T. Melai, Maastricht 2013

All rights reserved (including those of translation into other languages) to the author with exception of those chapters published by the journals referred to. No parts of this dissertation may be reproduced in any other form - by photo print, digital copy or any other means - without written permission from the author or the copyright owning journal. 


\title{
Plantar pressures in diabetic polyneuropathy
}

\author{
The influence of gait pattern \\ and exercise therapy
}

\section{Dissertation}

To obtain the degree of Doctor

at Maastricht University,

on the authority of the Rector Magnificus, Prof. dr. L.L.G. Soete, in accordance with the decision of the Board of Deans, to be defended in public on Friday 11 October 2013 at $14.00 \mathrm{hrs}$

by

\section{Tom Melai}




\section{Supervisor:}

Prof. dr. N.C. Schaper

\section{Co-supervisors:}

Dr. H.H.C.M. Savelberg

Dr. Ir. A.L.H. de Lange (Fontys University of Applied Sciences)

Dr. K. Meijer

\section{Assessment Committee:}

Prof. dr. M.K.C. Hesselink (chairman)

Prof. dr. R.A. de Bie

Dr. S.A. Bus (Academic Medical Center Amsterdam)

Prof. Dr. R.J.E.M. Smeets

Prof. Dr. B.M.P. Vanwanseele (Catholic University of Leuven)

The research presented in this dissertation was carried out at the Department of Human Movement Sciences, Maastricht University and at the Department of Allied Health Professions, Fontys University of Applied Sciences Eindhoven.

The research described in this dissertation was financially supported by Fontys University of Applied Sciences, ZonMW and the Dutch Diabetes Federation.

The reproduction of this dissertation was financially supported by Fontys University of Applied Sciences. 


\section{Contents}

Title and hyperlink

page

Chapter 1

General introduction

Chapter 2

The influence of gait on plantar pressures in diabetic

polyneuropathy: a review

Chapter 3

Chapter 4

Chapter 5

Chapter 6

Chapter 7

Appendix
Calculation of plantar pressure-time integral, an alternative approach

Increased forefoot loading is associated with an increased plantar flexion moment

Lower leg muscle strengthening does not redistribute plantar load in diabetic polyneuropathy. A randomized controlled trial Strength training affects lower extremity gait kinematics, not kinetics, in people with diabetic polyneuropathy

Summary and general discussion

Training program

Nederlandse samenvatting (Dutch summary)

Dankwoord (Acknowledgements)

Curriculum Vitae \& Publications 



\section{Chapter 1}

General Introduction 


\section{Diabetes}

Diabetes Mellitus is a metabolic disease that is characterized by elevated levels of blood glucose; a fasting plasma glucose level equal or higher than $7.0 \mathrm{mmol} / \mathrm{l}$ or a random plasma glucose equal or higher than $11.1 \mathrm{mmol} / \mathrm{l}[1,2]$. Glycosylated hemoglobin (HbA1c) provides an additional measure for glucose control over a longer period of time. The higher the percentage of the HbA1c, the higher the blood glucose has been in the last 2 to 3 months [3].

Several factors are responsible for these high levels of blood glucose. In type 1 diabetes there is a loss of insulin production, the hormone that regulates the blood glucose; this loss is usually caused by an autoimmune destruction of the insulin producing cells in the pancreas $[1,2$, 4]. In type 2 diabetes, insulin resistance is the most important factor responsible for high levels of blood glucose, causing a progressive diminished sensitivity of insulin receptors. But, in this type there is often a diminished insulin production as well. Type 2 diabetes is also associated with a marked increase in several cardiovascular risk factors such as obesity, hypertension and dyslipidemia $[1,2,4]$. There are also other types of diabetes, but these are less common.

Reducing the elevated blood glucose levels is one of the cornerstones in the treatment of diabetes. In type 1 , injections with insulin are necessary, as there is no production of this hormone. At the early stages of type 2 , treatment often consists of oral medication that improves the secretion of insulin or diminishes insulin resistance. At more advanced stages of type 2, also insulin injections might be used. For both types of diabetes also lifestyle changes are advised, as high body mass or sedentary behavior will further complicate the regulation of blood glucose [4].

In 2010, 285 million people were diagnosed with diabetes, resembling $6.4 \%$ of the world adult population [5]. The prevalence among adults is expected to increase to $7.7 \%$ in 2030 , resembling 439 million people worldwide as is shown in figure 1 [5]. In $201012 \%$ of the global health expenditure was used for diabetes and this is expected to increase with 30 to $34 \%$ in 2030 . These facts illustrate the enormous burden on global health and health care expenditures. It also stresses the importance of research to prevent or treat diabetes and its complications.
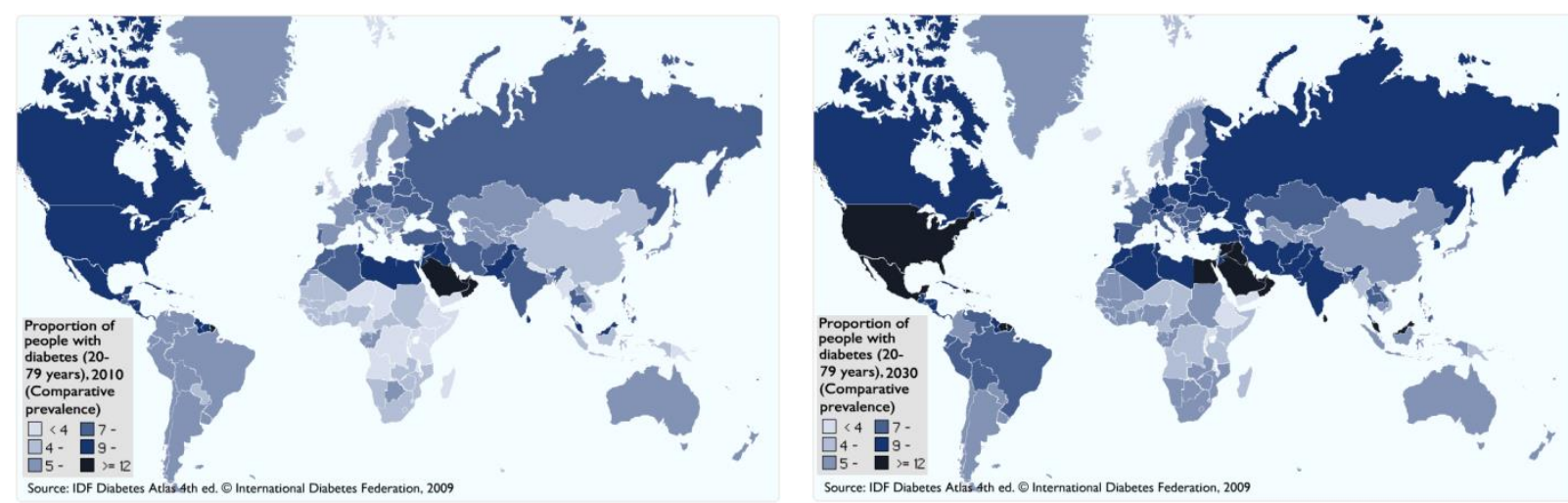

Figure 1. Proportion of people with diabetes worldwide aged between 20 and 79 years of age in 2010 (left) and 2030 (right)[5]. 


\section{Complications of diabetes}

The elevated blood glucose levels and alterations in cardiovascular risk factors can result in changes in large and small blood vessels, which are responsible for a high risk for complications in patients with diabetes. Alterations in large blood vessels contribute to coronary artery disease, stroke and peripheral vascular disease. Alterations in small blood vessels could lead to loss of vision (retinopathy), kidney failure (nephropathy) and peripheral nerve damage (polyneuropathy) [6].

Diabetic polyneuropathy (DPN) affects up to $40 \%$ of all patients with diabetes $[7,8]$ and can be classified as small and large fiber neuropathy. Nerve damage of small fibers can lead to symptoms such as burning superficial pain, impaired temperature sensation, reduced sensitivity and symptoms associated with autonomic neuropathy [9-11]. Large fiber damage can lead to symptoms such as impaired vibration perception and sensations, paresthesia, loss of tendon reflexes and loss of muscle strength [9-11]. Especially at more advanced stages of neuropathy the loss of muscle strength will become more prominent [12] and displays itself in alterations of the gait pattern $[13,14]$. Important factors that contribute to the development and progression of DPN are hypertension, smoking, elevated glycated hemoglobin levels, diabetes duration and a higher body mass index [15].

\section{Foot ulceration}

Diabetic polyneuropathy is a strong determinant for the occurrence of plantar ulceration [16], a complication in which local necrosis and inflammation result in wounds that are difficult to heal. Ulceration is responsible for $84 \%$ of the non-traumatic amputations of the lower limb [17], indicating the importance of treatment and prevention of this complication. Several factors have been identified as risk factor for the occurrence of ulceration; limitations in sensory feedback could lead to an inability to feel overloading of the plantar tissue [18], but an increase in plantar loading plays an important role as well [18]. Several studies indicated that plantar loading is increased in DPN [19], and even more that the location of high plantar loading is associated to the location of ulceration $[20,21]$.

\section{Plantar loading}

Plantar loading is influenced by the forces applied on the foot; the higher the force, the higher the load. But, loading also depends on the size of the area that these forces are applied to. Plantar loading is therefore often expressed as the quotient of force over contact area also known as pressure. Plantar pressures can be quantified by means of Pedobarography. In this technique sensors are used that cause a change in electric current under the influence of forces. Pressure can be calculated by taking the quotient of the measured forces over the surface of the sensors. For gait analysis two main applications of these sensors can be discerned; pressure insoles can be used to measuring the pressure interaction between foot sole and shoe during shod gait, and a pressureplatform can be used to measure the interaction of foot sole and surface during barefooted gait. For analysis of pressure data, the foot area is often divided in (anatomical) regions called masks. Per mask several parameters can be derived from sensor data. The most clinically used is peak 
pressure (PP), which is the highest pressure in a region. The pressure-time integral (PTI) provides information on the total amount of pressure a certain area is exposed to over time. The center of pressure (COP) describes the location of the average of all pressures under the foot at one time point.

\section{Prevention and treatment of a diabetic foot ulcer}

To prevent ulceration and its consequences, it is important to reduce high plantar pressures. Understanding the etiology of these high pressures is paramount to design successful interventions. According to Cavanagh et al. [22], 35\% of the variance in plantar pressures can be explained by structural factors, i.e. it is likely that other functional factors might be important contributors to these elevated pressures. Moreover, several studies have indicated that gait related factors play an important role as well and that DPN is associated with a progressive loss of muscle strength [23], which is suggested to be one of the factors responsible for increased plantar loading in DPN [24, 25]. However, the exact underlying mechanisms that lead to increased plantar pressures in patients with DPN are not fully understood, which complicates the prevention of high plantar pressures.

Current treatment consists mainly of offloading technology [26], such as shoe and insole adaptations, by means of which areas that are at risk or have been ulcerated can be relieved from high pressures. As this type of treatment does not interact with the underlying mechanisms responsible for these high pressures, it only treats the symptoms. Therefore, it is important that besides more knowledge on the underlying mechanisms responsible for increased forefoot loading in DPN, also more treatment options need to be explored to prevent the development of high plantar pressures.

\section{Aim of the dissertation}

The aim of the current dissertation is to contribute to the body of knowledge on the etiology of high plantar pressures in DPN, by providing more insight in the underlying gait related mechanisms responsible for increased plantar pressures in this population. A second aim is to evaluate the effects of a specific strength training program on the gait related factors that are considered to be responsible for increased plantar pressures in DPN.

\section{Outline of the dissertation.}

Chapter 2 (The influence of gait on plantar pressures in diabetic polyneuropathy: a review) is a narrative literature review on the underlying mechanisms of high plantar pressures in DPN. It shows that muscle activation, the control of ground reaction forces and joint moments, but also body mass and spatiotemporal gait characteristics have a considerable influence on plantar pressures.

Chapters 3 to 6 are based on a randomized controlled clinical trial (RCT). During analysis of baseline data of this trial we discovered that one of the plantar pressure parameters we planned to 
use was not valid; the pressure-time integral was not a true integral of pressure over time. In contrast, the force-time integral does provide a true integral, but does not account for the area it is applied to. As pressure equals the quotient of force over area, we were able to define and apply an alternative pressure-time integral. Chapter 3 (Calculation of plantar pressure-time integral, an alternative approach) explores the relevance of this new pressure-time integral in three groups; healthy elderly, diabetic controls and patients with diabetic polyneuropathy.

In chapter 4 (Increased forefoot loading is associated with an increased plantar flexion moment) we provided more insight in the biomechanics underlying increased forefoot loading by evaluating plantar pressures, spatiotemporal gait characteristics and joint moments simultaneously in previously mentioned three groups.

Chapter 5 (Lower Leg muscle strengthening does not redistribute plantar load in diabetic polyneuropathy. A randomized controlled trial) describes the effects of a specific strength training program on plantar pressure parameters in people with DPN.

Chapter 6 (Strength training affects lower extremity gait kinematics, not kinetics, in people with diabetic polyneuropathy) describes the effects of previously mentioned strength training program on joint moments and spatiotemporal gait characteristics in people with DPN.

Chapter 7 (Summary and general discussion) integrates all findings of previous chapters and discusses the strength and limitations of these findings in a clinical perspective. 


\section{References}

1. Alberti KG, Zimmet PZ: Definition, diagnosis and classification of diabetes mellitus and its complications. Part 1: diagnosis and classification of diabetes mellitus provisional report of a WHO consultation. Diabet Med 1998, 15(7):539-553.

2. Report of the expert committee on the diagnosis and classification of diabetes mellitus. Diabetes Care 2003, 26 Suppl 1:S5-20.

3. Higgins $\mathrm{T}: \mathrm{HbA}(1 \mathrm{c})$ for screening and diagnosis of diabetes mellitus. Endocrine 2012.

4. DeFronzo RA: International textbook of diabetes mellitus, 3rd ed / editors-in-chief, Ralph A. DeFronzo [et al.] edn. Chichester: Wiley; 2004.

5. Shaw JE, Sicree RA, Zimmet PZ: Global estimates of the prevalence of diabetes for 2010 and 2030. Diabetes Res Clin Pract 2010, 87(1):4-14.

6. Fowler M: Microvascular and macrovascular complications of diabetes. Clin Diabetes 2011, 29(3):116122.

7. Kumar S, Ashe HA, Parnell LN, Fernando DJ, Tsigos C, Young RJ, Ward JD, Boulton AJ: The prevalence of foot ulceration and its correlates in type 2 diabetic patients: a population-based study. Diabet Med 1994, 11(5):480-484.

8. Cabezas-Cerrato J: The prevalence of clinical diabetic polyneuropathy in Spain: a study in primary care and hospital clinic groups. Neuropathy Spanish Study Group of the Spanish Diabetes Society (SDS). Diabetologia 1998, 41(11):1263-1269.

9. Vinik AI, Mehrabyan A: Diabetic neuropathies. The Medical clinics of North America 2004, 88(4):947999, xi.

10. Scherens A, Maier C, Haussleiter IS, Schwenkreis P, Vlckova-Moravcova E, Baron R, Sommer C: Painful or painless lower limb dysesthesias are highly predictive of peripheral neuropathy: comparison of different diagnostic modalities. Eur J Pain 2009, 13(7):711-718.

11. Lavery LA, Peters EJG, Bush RL: High risk diabetic foot : treatment and prevention. New York: Informa Healthcare; 2010.

12. Andreassen CS, Jakobsen J, Andersen $\mathrm{H}$ : Muscle weakness: a progressive late complication in diabetic distal symmetric polyneuropathy. Diabetes 2006, 55(3):806-812.

13. Petrofsky J, Lee S, Bweir S: Gait characteristics in people with type 2 diabetes mellitus. Eur J Appl Physiol 2005, 93(5-6):640-647.

14. Katoulis EC, Ebdon-Parry M, Lanshammar H, Vileikyte L, Kulkarni J, Boulton AJ: Gait abnormalities in diabetic neuropathy. Diabetes Care 1997, 20(12):1904-1907.

15. Ziegler D, Gries FA, Spuler M, Lessmann F: The epidemiology of diabetic neuropathy. Diabetic Cardiovascular Autonomic Neuropathy Multicenter Study Group. J Diabetes Complications 1992, 6(1):49-57.

16. Young MJ, Breddy JL, Veves A, Boulton AJ: The prediction of diabetic neuropathic foot ulceration using vibration perception thresholds. A prospective study. Diabetes Care 1994, 17(6):557-560.

17. Pecoraro RE, Reiber GE, Burgess EM: Pathways to diabetic limb amputation. Basis for prevention. Diabetes Care 1990, 13(5):513-521.

18. Birke JA, Patout CA, Jr., Foto JG: Factors associated with ulceration and amputation in the neuropathic foot. J Orthop Sports Phys Ther 2000, 30(2):91-97.

19. Pitei DL, Lord M, Foster A, Wilson S, Watkins PJ, Edmonds ME: Plantar pressures are elevated in the neuroischemic and the neuropathic diabetic foot. Diabetes Care 1999, 22(12):1966-1970.

20. Caselli A, Pham H, Giurini JM, Armstrong DG, Veves A: The forefoot-to-rearfoot plantar pressure ratio is increased in severe diabetic neuropathy and can predict foot ulceration. Diabetes Care 2002, 25(6):1066-1071.

21. Mueller MJ, Zou D, Bohnert KL, Tuttle LJ, Sinacore DR: Plantar stresses on the neuropathic foot during barefoot walking. Phys Ther 2008, 88(11):1375-1384.

22. Cavanagh PR, Morag E, Boulton AJ, Young MJ, Deffner KT, Pammer SE: The relationship of static foot structure to dynamic foot function. J Biomech 1997, 30(3):243-250.

23. Andersen $\mathrm{H}$, Poulsen $\mathrm{PL}$, Mogensen $\mathrm{CE}$, Jakobsen $\mathrm{J}$ : Isokinetic muscle strength in long-term IDDM patients in relation to diabetic complications. Diabetes 1996, 45(4):440-445.

24. Abboud RJ, Rowley DI, Newton RW: Lower limb muscle dysfunction may contribute to foot ulceration in diabetic patients. Clin Biomech (Bristol, Avon) 2000, 15(1):37-45.

25. Savelberg HH, Schaper NC, Willems PJ, de Lange TL, Meijer K: Redistribution of joint moments is associated with changed plantar pressure in diabetic polyneuropathy. BMC Musculoskelet Disord 2009, $10: 16$.

26. Bus SA: Priorities in offloading the diabetic foot. Diabetes Metab Res Rev 2012, 28 Suppl 1:54-59. 


\section{Chapter 2}

The influence of gait on plantar pressures in diabetic polyneuropathy: a review 


\begin{abstract}
High plantar pressures, often expressed in peak pressures or pressure-time integrals, increase the risk of ulceration for people with diabetic polyneuropathy. The cascade of changes in the lower leg and foot leading from diabetic polyneuropathy to gait alterations to increased loading of the plantar area is however not fully understood. In this review we describe how muscle activation, joint moments and ground reaction forces affect the plantar pressures of people with diabetic polyneuropathy. Analysis of literature revealed changes in activation patterns of the plantar and dorsal flexors, but the cascade through which plantar pressures are affected is still unclear. In diabetic polyneuropathy, spatiotemporal gait characteristics, such as gait velocity and stride length are affected and can have large effects on plantar pressures. Hence, these gait characteristics must be monitored or standardized during plantar pressure measurements. There is some evidence that a slower gait velocity and smaller stride length can be used to lower plantar pressures and thereby decrease the risk for ulceration. In addition, other strategies, such as using walking aids or dividing the time spent walking evenly over the day could lead to lower plantar pressures and therefore could enable an increase in daily activity, without increasing the risk of excessive plantar loading. The findings in this review stress the importance for gait related interventions to reduce increased plantar loading in diabetic patients with polyneuropathy.
\end{abstract}




\section{Introduction}

Foot ulceration is a feared complication of diabetes mellitus, resulting in a high burden of disease and high costs. The worldwide prevalence of diabetes in adults will increase up to $7.7 \%$ by the year 2030 [1]; measures need to be taken to limit the rise of patients with a foot ulcer. Several factors are involved in the development of a diabetic foot ulcer but distal symmetrical diabetic polyneuropathy (DPN) plays a central role in most patients [2,3]. DPN is a complication that occurs in 20 to $40 \%$ of all patients with diabetes [4, 5]. Loss of protective sensation and increased biomechanical stress, such as increased plantar pressures, play a central role in the pathways to neuropathic ulceration. Various mechanisms are involved in the rise of plantar pressures in diabetic neuropathy, in this review we will focus on the role of alterations in lower limb biomechanics, an area that until recently received little attention.

DPN leads to sensory nerve malfunctioning and due to the loss of protective sensation (micro) traumas are not adequately sensed [6]. In addition to the impaired ability to sense biomechanical overloading of the plantar aspect of the foot, DPN can also contribute to this tissue overloading via several mechanisms. Whether tissue is or is not overloaded depends on the balance between load and load-ability; both can be affected by DPN [7-9]. As reviewed elsewhere [10] load-ability can be influenced by a disruption of physiological processes in the skin and underlying tissues due to the loss of nerve function; for example, impaired microvascular perfusion could lead to a decreased nourishment of plantar tissue and consequently a diminished load-ability [9].

An increase in loading depends on the magnitude of the loading force and the size of the area the load is applied to; the smaller the area, the larger the effects of the forces. Therefore, plantar loading is often expressed as the quotient of force over contact area, also known as plantar pressure, which can be measured by having subjects walk over a pressure-platform. Plantar loading can be quantified by several parameters, of which peak pressure is the most commonly used, describing the highest pressure under the foot or in a certain plantar area. The pressure-time integral of the foot, another pressure-related parameter that is commonly used, is a measure for the total pressure exposure of a certain plantar area during a step. In DPN, especially the forefoot seems to be prone to high peak pressures and high pressure-time integrals, both leading to subsequent ulceration $[7,11]$.

Several factors are responsible for the high plantar pressures observed in DPN. Anatomical alterations such as bone deformation, soft tissue alterations and impaired range of joint motion can have negative effects on plantar pressures. How these alterations influence plantar pressures has previously been described in other reviews $[6,12]$, but the series of events leading from DPN to gait alterations to increased loading of the plantar area is not yet fully understood. Two reviews clearly described the effects of DPN on the gait pattern, but did not address the underlying mechanisms responsible for increased plantar loading in this population [13, 14]. Three other reviews addressed the factors responsible for increased plantar loading, but were focused on shoe and offloading technology [15-17]. Therefore, the aim of the current narrative review was to provide an overview of how changes in gait pattern due to DPN can contribute to adverse plantar pressure patterns and even more, to explore if strategies that manipulate the gait pattern can be used to lower plantar pressures in this population. 


\section{Material and Methods}

To provide structure in literature, a theoretical model was constructed [1-17] of the pathways leading from diabetic polyneuropathy through muscle function and gait dynamics to plantar loading (figure 1). Gait dynamics was defined as all forces, joint moments and spatiotemporal gait characteristics that could interfere with the gait patterns and plantar loading.

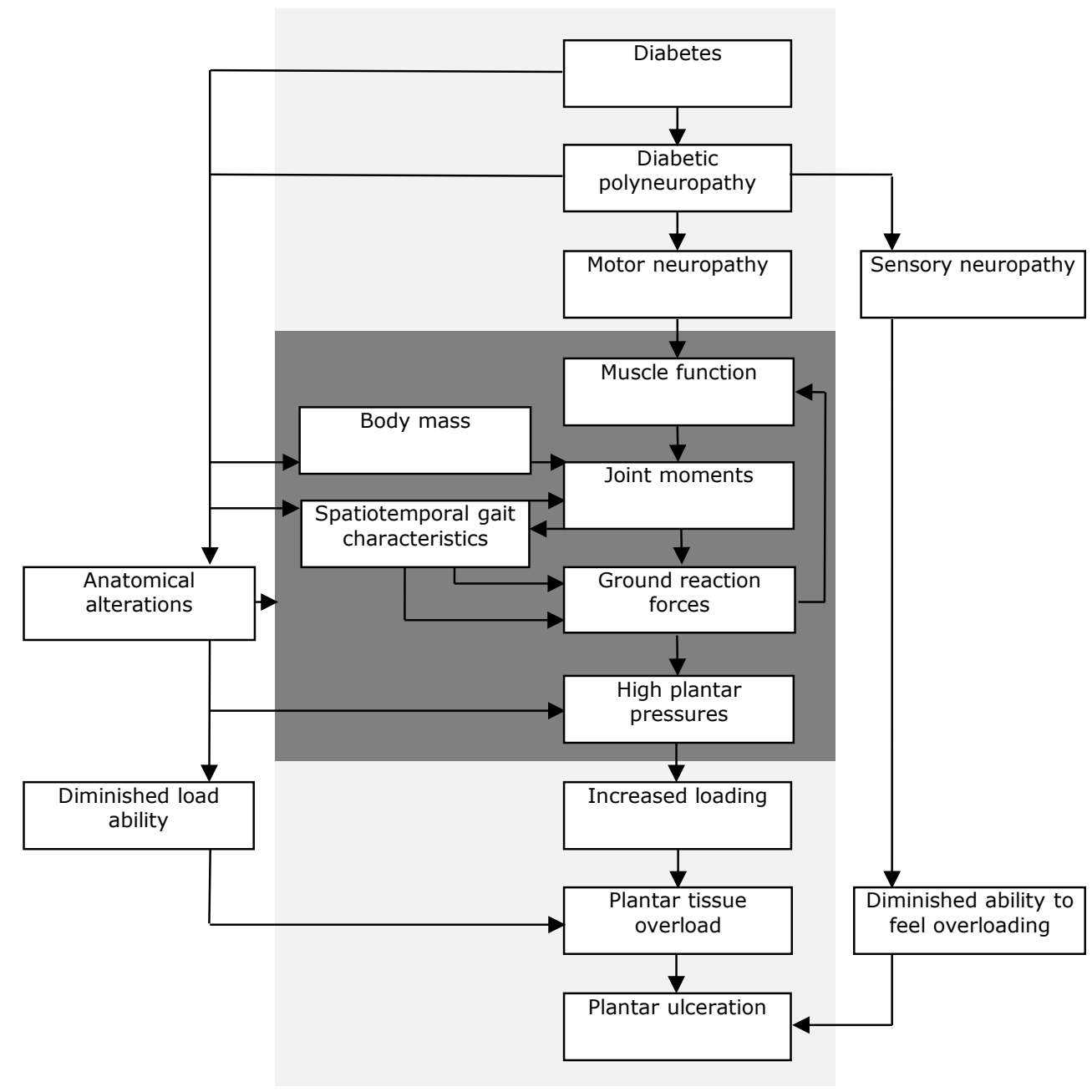

Figure 1. A theoretical model of the pathways leading from diabetic polyneuropathy through muscle function and gait dynamics to plantar loading.

An electronic search was performed in Pubmed in March 2012 to find all articles addressing the underlying mechanisms leading to increased plantar loading and the influence of diabetes. The keywords used were: ((plantar pressure) OR (plantar pressures) OR (plantar loading)) OR ((foot OR ankle) AND (strength OR torque OR force OR moment OR dynamics) AND (diabetes OR diabetic)). The articles were limited to those with the keywords reported in the title or abstract, those written in English language and those that included human adult subjects. A first selection of the articles was made based on titles and abstracts. Articles were excluded if the main topic did not address plantar pressures, force dynamics during gait, the mechanisms underlying increased plantar pressures, force dynamics of the lower extremity or were reviews. Studies on the effects of sports or running, shoe/ offloading technology, passive structures, limited joint mobility, cutaneous 
sensation, medication, surgical interventions or methodological matters were also excluded from further reviewing. The articles included in this narrative review studied diabetic patients with or without polyneuropathy or non-diabetic subjects with polyneuropathy. Studies were excluded if subjects had any additional pathology that could affect plantar loading or force dynamics like fractures, tendinopathy or previous amputations.

The search resulted in 1224 articles. Of these articles 49 were included and of 1 article the full text could not be obtained. Data synthesis was not possible due to the large heterogeneity of the studies.

\section{Changes in muscle function affect plantar loading during gait in diabetic polyneuropathy}

In the case of DPN, sensor and motor nerves are affected, which can lead to impaired force generation during gait (as discussed in a paragraph below) and impaired sensorimotor feedback that will affect motor control and plantar pressures. The impaired sensorimotor feedback could affect plantar pressures through alterations in muscle activation and deactivation, which can be measured in gait studies by means of portable electromyography. Indeed, the activity of the plantar flexors influences plantar loading. In a cadaver study the loss of activity of the hallucis longus and flexor digitorum longus was simulated, which resulted in a smaller contact area and less loading of the toes, and increased contact area and loading of the forefoot [18]. In DPN patients an abnormal activation pattern of the plantar flexors has been observed concurring with a faster rolloff of the foot and higher forefoot loading. In particular, the soleus muscle is earlier activated and later deactivated [19], the medial gastrocnemius muscle is earlier activated [20] and the lateral gastrocnemius has a later peak occurrence [19]. One study [21] observed correlations of more than 0.87 for the time of onset of activation of the medial gastrocnemius and the magnitude of peak pressures underneath the forefoot and toes of healthy subjects. This study also observed that the amplitude of the peak of this activation pattern was correlated $(r \geq 0.90)$ to the peak pressure under the medial forefoot, toes and heel [21].

Reduced force generation or a delayed activation of the dorsal flexors can also lead to impairments in braking the roll-off of the foot, resulting in increased forefoot pressures. Peak amplitude [22] and peak time occurrence [22, 23] of tibial anterior muscle activation were unaltered in DPN. The activation of this muscle is however prolonged by more than 5\% [20, 24] suggesting an impaired motor control of the roll-off of the foot. Moreover, a delayed neural activation of this muscle, which probably results in a delayed rate of force development, is associated with earlier forefoot loading [25]. Besides changes in the activation of the plantar and dorsal flexors, the activation of the knee extensors and flexors is also affected in DPN. The knee extensors are approximately 10\% longer activated [24], but contradictory data has been published on the peak occurrence of these muscles in DPN $[19,23]$. The hamstrings show earlier activation and delayed deactivation in this population [20]. It is unclear whether these changes in activation of the knee flexors and extensors are a direct effect of DPN, as these changes can be part of a gait pattern to compensate limitations of other muscles. It is also unclear how the knee flexors and extensors influence functioning of the plantar and dorsal flexors. The aforementioned studies point 
to a role of altered neural activation of the lower limb muscles in elevated plantar pressure in DPN, the role of the upper leg muscles remains to be determined.

\section{Ground reaction forces are affected by diabetic polyneuropathy}

During gait, we exert forces on the ground and as a reaction the ground will exert an equal force on our body, which is called the ground reaction force (GRF, figure 2a). By regulating our muscle activity we can control the force applied to the ground and thus manipulate the GRF. As DPN can affect muscle activity, it can affect the forces exerted on the ground and consequently the GRF. In the sagittal plane the GRF is divided in two force components, the vertical and horizontal component (figure 2a). As pressure is the quotient of force and the area it is exerted on, a higher vertical component of the GRF will result in higher plantar pressures. Changes in the amplitude or direction of the horizontal component affect the forward displacement of the point of application of the GRF and thus determine which part of the foot is loaded and what the timing of the loading is.

In DPN both the vertical and the horizontal (for-aft) component of the GRF are affected. Intuitively, one would expect higher GRFs in DPN patients as plantar pressures are also higher, but results of different studies are contradictive. A lower peak vertical component of the GRFs in DPN was reported in four studies [19, 26-28], but no significant differences were observed in four other studies [29-32]. These contradictory findings could be explained by several confounders. In most of aforementioned studies [19, 26, 27, 29-31] gait velocity was not standardized and subjects were allowed to choose their own gait velocity, which could have introduced more variation between groups as will be discussed below. In addition, DPN patients prefer to walk slower with less vertical acceleration, resulting in lower GRFs [33]. Another important confounder for the GRF is body mass, with a higher body mass, higher forces are exerted on the ground, resulting in higher GRFs. One study demonstrated the effect of gait velocity and body mass on the GRF in diabetes. Initially lower vertical GRFs were observed in DPN, when patients walked at their preferred gait velocity. But, when the control group was matched for gait velocity and outcome was expressed as a percentage of body mass, DPN patients had a higher vertical GRF [28]. These findings stress the importance of monitoring or controlling gait velocity and body mass when comparing forces and pressures. In addition, it is currently unclear how the vertical component of the GRF is affected by DPN.

Studies on the effect of DPN on the horizontal component of the GRF are more in agreement. The majority of studies, except one [30], reported a decrease of horizontal GRF during preferred [27, 31] and standardized gait velocity [32], indicating diminished braking and propulsion during the stance phase. These changes in braking and propulsion could influence the displacement of the center of pressure [32], thereby altering plantar pressure patterns over time [34]. However, only few authors studied the relationship between the GRF and plantar pressures. These measurements require a more complex experimental set-up as a pressure-platform needs to be mounted on top of a force-platform, but these data need to be obtained in order to unravel how the different forces on the foot affect plantar pressures in DPN. 
$2 a$

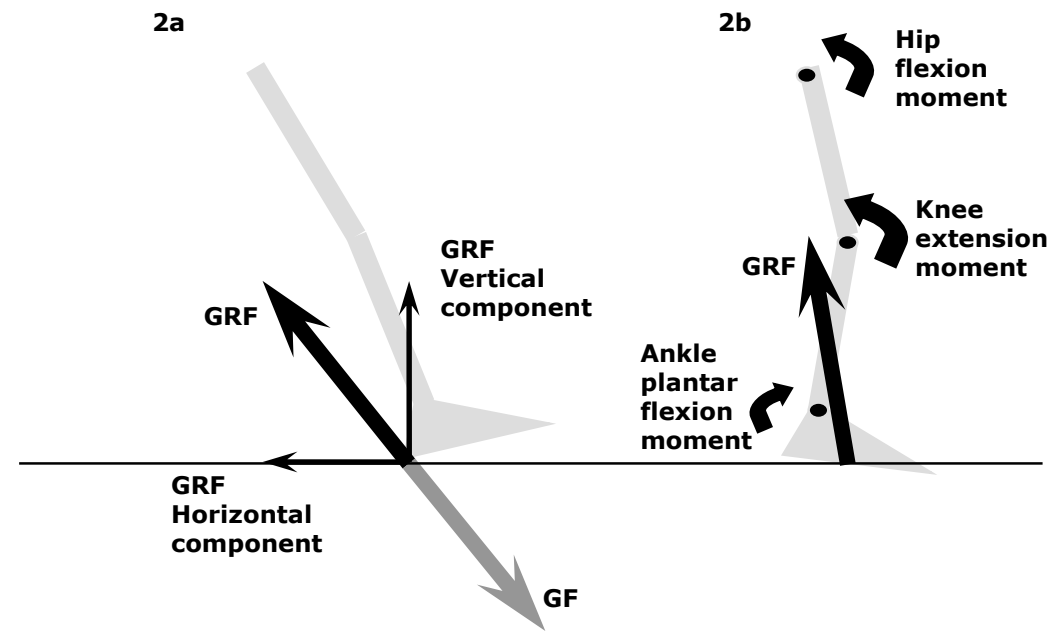

Figure 2. The ground reaction force (2a) and joint moments (2b) during gait (for the sake of clarity joint moments are not depicted in 2a). The ground reaction force (GRF) is the resultant of the forces that the ground exerts on a body as a reaction to the forces we exert on the ground (GF). The GRF can cause rotary movements in the joints of the lower extremity. In this example the GRF will cause dorsal, knee and hip flexion. To remain in balance, the body needs to counteract these movements by creating rotatory movements in the opposite direction. In this example the body needs a plantar flexion, knee extension and hip flexion moment, to remain in balance.

\section{Increased body mass leads to higher plantar pressures}

As discussed previously, a higher body mass affects the GRF. When an increase in body mass is simulated by having healthy subjects wear extra mass, the peak plantar pressure rises, and an increase of $9.1 \mathrm{~kg}$ results in an increase of peak pressure of 5.0 to $14.3 \%$ [35]. The largest effects of obesity on plantar pressures are found in the mid-foot [36-38] and metatarsal heads [36, 37]. But, when plantar pressures are normalized for body mass, only the pressure under the mid-foot remains increased in obese subjects [37]. It is conceivable that this increase of mid-foot loading is the result of functional adaptations such as sagging of the longitudinal arch resulting in a larger area of support. On the other hand the ability to control the roll-off of the foot seems to be intact in obese subjects [39]. The contribution of body mass to plantar pressures has been quantified by several studies. The variation in body mass explains less than $40 \%$ of the variance in peak plantar pressure in healthy subjects [40-43]. In DPN patients the effect of body mass on peak pressures is less, in one study the variation in body mass could explain $14 \%$ of the variance in peak pressure [44] while in another study no effect of body mass was found in stepwise regression models [45], probably due to factors such as anatomical deformities and diminished motor control. Based on these studies, it is likely that weight loss is not an effective strategy to reduce plantar pressures in subjects with DPN.

\section{Joint moments influence plantar pressures}

DPN related changes in muscle forces will influence the GRF. The rotatory effects of these muscle forces about the joints can be expressed as joint moments (figure 2b). For example, an increase of force generated by the plantar flexors will lead to a higher plantar flexion moment, which will result in higher forces exerted on the ground and consequently a higher GRF. Vice versa, the magnitude 
and orientation of the GRF influence the joint moments as well. If the body is in balance, than all forces acting upon the body must be in balance as well and a rise in GRF needs to be compensated by an increase in joint moments. Therefore, an approximation of joint moments can be obtained from GRF measurements using a force-platform in combination with measurements of the orientation and acceleration of body segments during gait using video [46].

The joint moments of knee and hip are probably not affected by DPN [29, 31, 32]. In contrast, the maximum ankle plantar flexion moment is lower in DPN [29, 31], but when gait velocity is standardized these differences disappear [32]. At a standardized gait velocity an increase of the ankle plantar flexion moment was observed at $40 \%$ of the ground contact of the foot (stance phase), which was hypothesized to be the result of an earlier forward displacement of the point of application of the GRF which was thought to result from a diminished ability of the dorsal foot flexors to brake the forward velocity.

Joint power, the product of joint moments and angular velocity, is another quantity that can be used to express the forces transmitted through the lower extremity. During walking the plantar flexors are used to propel the body forward and the hip flexors are used to raise the leg. In DPN, the plantar flexion joint power is decreased $[29,31]$ and the hip flexion power is increased during late stance phase [31], compared to healthy subjects. Based on these results it was hypothesized that DPN patients use hip flexion to pull their leg forward in late stance phase, instead of pushing forward with their plantar flexors [31]. What caused the differences in joint power is unclear, but it is conceivable that diminished force generation plays a central role. Future research should further explore previously mentioned hypotheses and further define the determinants of joint power and joint moments.

\section{The effects of spatiotemporal gait characteristics on plantar pressures}

Joint moments, ground reaction forces and also spatiotemporal gait characteristics, such as gait velocity, stride length, stride time, stance phase duration (the duration of unilateral ground contact) and cadence (the number of steps per minute), are all interrelated. Therefore, changes in these gait characteristics, which are common in DPN, are likely to affect or result from changes in joint moments and ground reaction forces and are likely to concur with changes in plantar pressures.

Gait velocity is an important determinant of plantar pressure and can be affected by DPN. A lower gait velocity is coupled with less vertical acceleration of the body and consequently with reduced plantar peak pressures. Published values for the preferred gait velocity in healthy subjects range from 1.06 to $1.34 \mathrm{~m} / \mathrm{s}$ and for people with diabetes from 0.74 to $1.15 \mathrm{~m} / \mathrm{s}$ [24, 28, 29, 4749]. The presence of peripheral nerve damage can lead to a further reduction in gait velocity, especially in more advanced stages $[24,28,47,50]$. The effect of gait velocity on peak pressures becomes clear when gait velocity is increased from 0.75 to $2.00 \mathrm{~m} / \mathrm{s}$ in healthy elderly, leading to a rise of peak pressure in the different plantar areas between 9\% (lateral forefoot) to $90 \%$ (heel) [51], with a linear increase in the areas under the hallux, heel [51] and the medial forefoot [21]. At gait velocities above $1.5 \mathrm{~m} / \mathrm{s}$ different areas are loaded differently; where the heel and hallux still show a linear increase, other areas show a decrease of peak pressure [51]. Peak pressures also 
rise under the medial metatarsal heads and toes with increasing gait velocity [52], but under the mid-foot and proximal forefoot the peak pressure decreases [53].

The pressure-time integral, is also affected by a faster gait velocity probably due to shorter loading periods of plantar areas. Indeed, when gait velocity increases, the pressure-time integral decreases under the heel, mid-foot and metatarsal heads [21, 52]. When DPN was simulated by cooling down the feet of healthy volunteers with ice water [54], a lower gait velocity was observed with an increased pressure-time integral. So with decreasing gait velocity the peak pressures decrease, while the pressure-time integral increases. In contrast, a faster gait velocity will result in higher peak pressures, but lower pressure-time integrals. However, it is unclear, whether gait velocity affects the pressure-time integral linearly and whether regions are affected differently at higher gait velocities. Furthermore, as aforementioned studies on the effects of gait velocity on plantar pressures were performed in healthy subjects, it would be interesting to know how these relationships are affected by the presence of DPN. As previous studies do show that plantar pressures are affected by gait velocity, it is advisable for future studies to at least monitor gait velocity, but preferably to standardize it.

Also stride length is reduced for people with DPN and can influence plantar loading. Stride lengths range from 1.14 to $1.4 \mathrm{~m}$ for healthy subjects, from 1.22 to $1.26 \mathrm{~m}$ for diabetic subjects without neuropathy and from 1.15 to $1.20 \mathrm{~m}$ for DPN [29, 32, 48]. But, when patients with diabetes are asked to walk at the same gait velocity as age matched controls, these differences in stride length disappear [24] and the differences in stride length observed in people with diabetes seem to be a result of a slower gait velocity. Nevertheless, when gait velocity is kept constant, but stride length is decreased by $20 \%$, the ankle joint moment decreases by $5 \%$, and the hip joint moment increases by $28 \%$. Even more, this decrease in stride length will lead to a lower peak pressure under the heel, toes and mid-foot of approximately $15 \%$ and also a lowered pressure-time integral of 20 to $60 \%$ for the metatarsal heads, heel and hallux [55]. In the aforementioned studies measurements were performed during a single step. As during gait the length of each step can vary, future research on the effects of varying stride length should monitor plantar pressures over a defined distance containing several steps, instead of a single step. Moreover, plantar pressures are usually measured in a gait lab in subjects who are unfamiliar with the test settings resulting in a less confident gait pattern with reduced stride length, subsequently leading to lower plantar pressures and an underestimation of plantar loading in these subjects in daily life. Repeating these measurements will enable subjects to familiarize with the measurements; indeed we recently observed that during repeated measurements over time in a gait lab, stride length and gait velocity increased in DPN patients (Melai et al., unpublished observations).

Cadence (i.e. the number of steps per minute) is another gait characteristic influencing plantar pressure. Cadence in combination with body mass can explain up to $23 \%$ of the variance in peak pressure and $24 \%$ of the variance in mean plantar pressure in healthy subjects [40]; this shows that the number of steps per minute influences the plantar load of a single step. Even more, taking fewer steps will reduce the cumulative load. In diabetic subjects the cadence is reduced but no difference was observed between subjects with and without DPN [29]. Combining two studies, healthy volunteers take 103.5 to 106.8 steps per minute, diabetic subjects take approximately 101.2 steps and DPN patients take 99.4 to 100 steps per minute $[23,29]$. As an increase from 70 to 120 steps per minute leads to an increase of 119 and $45 \%$ of the peak pressure and pressure- 
time integral respectively [56], differences in cadence should be taken into account or standardized during plantar pressure measurements in people with diabetes.

Stance phase duration and stride time are affected as well in diabetes. Average stance phase duration during preferred gait velocity ranges from 0.50 to $0.61 \mathrm{~s}$ in healthy elderly, from 0.62 to $0.66 \mathrm{~s}$ for people with diabetes and from 0.64 to $0.73 \mathrm{~s}$ in DPN [24, 48]. Average stride times range from 1.10 to 1.40 for healthy elderly, 1.13 to 1.20 for people with diabetes and 1.13 to 1.23 for people with DPN $[24,26,29,48]$. It is conceivable that longer stance phase durations and stride times will result in longer loading of plantar areas and consequently higher pressure-time integrals. But, the exact effects of changes in stance phase duration and stride time on plantar pressures has not yet been described in the literature and should be further explored.

\section{Effects of interventions on plantar pressures}

Various studies reported that plantar pressures can be lowered by changing the gait pattern in both diabetic and non-diabetic subjects. Patients at risk for increased loading can be asked or taught to change their spatiotemporal gait characteristics. A reduction in gait velocity is an intervention that is easy to apply and will lead to lower peak pressures [21, 51]. Unfortunately reducing gait velocity could also lead to higher pressure-time integrals, which might contribute to tissue damage as well. Currently, it is unclear how high peak pressures and high pressure-time integrals interact in causing plantar tissue damage. Intuitively one would advise diabetic patients with elevated plantar pressures to walk slower, but given the contradictory effect on peak pressure and pressure-time integral, it is currently unclear which gait velocity would be optimal. Another advice that can be given to patients with high plantar pressures is to reduce their stride length. Even when gait velocity is kept constant, a reduction in stride length will lead to a reduction in both peak pressures and pressure-time integrals $[55,57]$. Therefore, reducing stride length can be used to lower loading per step. Although the effects per step are promising, it should be realized that in order to reduce stride length more steps must be taken to cover the same distance, which will affect cumulative loading.

Other interventions to change gait strategies have shown to be successful as well. When healthy subjects change their regular gait pattern to shuffling, peak pressure is reduced with $41.6 \%$; the pressure-time integral is lower under the metatarsal heads and the hallux, but rises with $78 \%$ under the heel [58]. Also a hip strategy is successful in reducing peak pressures in the forefoot [59]. With this strategy subjects reduced the push-off in late stance phase, but pull their leg forward from the hips, without changing their gait velocity, resulting in a decrease of forefoot peak pressure of $27 \%$, but an increase in pressure under the heel of $24 \%$ [59]. Intuitively, one would expect that learning a new gait pattern is very difficult to achieve, but in another study subjects were trained during a day and after one week without further training they were able to reproduce the aforementioned hip strategy in a laboratory setting [60]. However, it remains to be determined if such newly learned gait strategies can be implemented in daily life.

Another strategy that seems to be successful is step-to-gait. In this gait pattern, one foot (leading foot) is placed forward as in regular gait, but the other foot (trailing foot) is placed next to the first one. Step length and stride length will therefore be equal. In healthy subjects, this 
strategy leads to a reduction in peak pressure of both the heel and the central metatarsal heads of respectively 9 and $67 \%$ in the leading foot and 32 and $19 \%$ in the trailing foot [57], but this reduction in peak pressure is at least partly contributed to a reduction to half of the gait velocity during regular gait. When gait velocity is unchanged, then such a gait strategy will lead to a decrease of peak pressures of 53\% underneath the forefoot and an increase of $14 \%$ underneath the heel [61]. In tests with a few number of DPN patients similar results were found [57, 61].

Also walking aids could be used in offloading the plantar area, and walking with two poles as during Nordic walking can be successful in reducing plantar pressures [62]. In people without experience in Nordic walking, loading of the central metatarsal heads can decrease by $32 \%$, when walking at preferred gait velocity. In more experienced people, central metatarsal head loading can decrease up to $37 \%$ while walking at preferred gait velocity, and $50 \%$ while walking $20 \%$ faster. In contrast to these results, the use of a single cane does not affect plantar pressures [61]. Increasing physical activity is one of the cornerstones in the treatment of diabetes and in the elderly diabetic population walking is the most important daily physical activity. In a population based pedometer study, DPN patients walked less compared to diabetic subjects without DPN [63]. Nordic walking could therefore be a useful strategy to increase daily activity without increasing the risk of excessive plantar loading.

Besides the aforementioned changes in gait patterns, strength training could also affect plantar loading. As discussed in this review the force generating capacity of the lower limb muscles play an important role in loading of the forefoot. But, only a few studies have been performed that tried to alter plantar loading by increasing muscle force and motor control [64, 65]. A reduction in peak pressure [64] and spatiotemporal differences in plantar loading [65] have been reported as an effect of a one year exercise program in postmenopausal women. But, the exact effects of the exercise program on the control of the roll-off of the foot are not clear.

In our experience diabetic patients with DPN can have complaints of increased fatigability of legs. The effect of muscle fatigability on plantar pressures was tested by two studies [66, 67]. When healthy subjects were exposed to long distance walking over several days, they increased their contact time, mean pressure, peak pressure and pressure-time integral under the heel and forefoot, but experienced less pressure under the toes [66]. A 30 minutes run, results in a $10 \%$ increase of plantar pressure under the second and third metatarsal heads, and a $8 \%$ decrease of heel loading [67]. A decrease in motor control due to muscle fatigue can lead to increased forefoot loading, therefore it seems advisable that DPN patients with elevated plantar pressures do not walk in bouts of long duration, but preferably they should divide the time spent walking evenly. The role of muscle fatigability needs to be confirmed, for instance one would expect deterioration of plantar pressure patterns when exposing DPN patients to fatiguing exercise. Vice versa, strength training could reduce the muscle fatigability in DPN with subsequent beneficial effects on plantar pressure.

\section{Discussion}

A variety of studies have been performed that provided more insight in how changes in gait pattern can contribute to adverse plantar pressure patterns in patients with DPN. But, based on current literature the series of events leading to higher plantar pressures cannot yet be identified, as there 
are contradictive results on the contribution of lower limb muscle function, force generation and ground reaction forces to plantar pressures. These contradictions might be the result of large varieties in subject characteristics, gait patterns and test settings. The presence or severity of polyneuropathy is not always reported and subjects are often tested at their preferred gait velocity, which can introduce bias and increase the variance of outcome measures. In addition, several studies reporting on gait related mechanisms were probably not performed with a sufficient number of subjects, which may lead to insufficient power. An instrumented gait analyses can be time consuming, but we believe that by using current available laboratory settings it is possible to obtain more clarity on above mentioned mechanisms leading to increased plantar pressures. To better understand the clinical effect of plantar pressures in DPN and to render studies comparable, it is important to monitor or standardize gait characteristics and other confounders. It is clear that answering our questions on how gait related mechanisms are responsible for increased plantar pressures demand more research and especially under controlled conditions and with a large number of subjects.

In conclusion, impaired function of the dorsal and plantar flexors plays an important role in the forward displacement of center of pressure in DPN and consequently results in a higher loading of the forefoot. Alterations in gait characteristics, such as a slower gait velocity or smaller steps, can result in lower peak pressures and can therefore be used in the prevention and treatment of patients at risk for ulceration. Other gait strategies in order to prevent muscle fatigue, such as using walking aids, or dividing the time spent walking evenly over the day could also be useful to increase daily activity, without increasing the risk of excessive plantar loading. A reduction of body mass will also lead to a reduction of plantar pressures, but as its contribution to plantar pressures is relatively low, it is not expected that weight loss is an effective strategy to prevent abnormal loading of the foot. Reduction of the abnormal loading of the foot is one of the cornerstones in the prevention of diabetic foot ulcers and currently most interventions are focused on shoe wear and insoles. The current review stresses that changes in gait pattern can also have considerable effects on plantar pressures and interventions to alter gait characteristics in subjects with DPN could be an alternative approach to reduce the abnormal loading of the foot in DPN. 


\section{References}

1. Shaw JE, Sicree RA, Zimmet PZ: Global estimates of the prevalence of diabetes for 2010 and 2030. Diabetes Res Clin Pract 2010, 87(1):4-14.

2. Pecoraro RE, Reiber GE, Burgess EM: Pathways to diabetic limb amputation. Basis for prevention. Diabetes Care 1990, 13(5):513-521.

3. Crawford F, Inkster M, Kleijnen J, Fahey T: Predicting foot ulcers in patients with diabetes: a systematic review and meta-analysis. QJM : monthly journal of the Association of Physicians 2007, $100(2): 65-86$.

4. Kumar S, Ashe HA, Parnell LN, Fernando DJ, Tsigos C, Young RJ, Ward JD, Boulton AJ: The prevalence of foot ulceration and its correlates in type 2 diabetic patients: a population-based study. Diabet Med 1994, 11(5):480-484.

5. Cabezas-Cerrato J: The prevalence of clinical diabetic polyneuropathy in Spain: a study in primary care and hospital clinic groups. Neuropathy Spanish Study Group of the Spanish Diabetes Society (SDS). Diabetologia 1998, 41(11):1263-1269.

6. Boulton AJ: The diabetic foot: from art to science. The 18th Camillo Golgi lecture. Diabetologia 2004, 47(8):1343-1353.

7. Caselli A, Pham H, Giurini JM, Armstrong DG, Veves A: The forefoot-to-rearfoot plantar pressure ratio is increased in severe diabetic neuropathy and can predict foot ulceration. Diabetes Care 2002, 25(6):1066-1071.

8. Mueller MJ, Zou D, Bohnert KL, Tuttle LJ, Sinacore DR: Plantar stresses on the neuropathic foot during barefoot walking. Phys Ther 2008, 88(11):1375-1384.

9. Schaper NC, Huijberts M, Pickwell K: Neurovascular control and neurogenic inflammation in diabetes. Diabetes Metab Res Rev 2008, 24 Suppl 1:S40-44.

10. Schaper NC, Havekes B: Diabetes: impaired damage control. Diabetologia 2012, 55(1):18-20.

11. Guldemond NA, Leffers P, Walenkamp GH, Schaper NC, Sanders AP, Nieman FH, van Rhijn LW: Prediction of peak pressure from clinical and radiological measurements in patients with diabetes. $B M C$ Endocr Disord 2008, 8:16.

12. Bus SA: Foot structure and footwear prescription in diabetes mellitus. Diabetes Metab Res Rev 2008, 24 Suppl 1:S90-95.

13. Wrobel JS, Najafi B: Diabetic foot biomechanics and gait dysfunction. J Diabetes Sci Technol 2010, 4(4):833-845.

14. Allet L, Armand S, Golay A, Monnin D, de Bie RA, de Bruin ED: Gait characteristics of diabetic patients: a systematic review. Diabetes Metab Res Rev 2008, 24(3):173-191.

15. Bowling FL, Reeves ND, Boulton AJ: Gait-related strategies for the prevention of plantar ulcer development in the high risk foot. Current diabetes reviews 2011, 7(3):159-163.

16. van Schie $\mathrm{CH}$ : A review of the biomechanics of the diabetic foot. Int J Low Extrem Wounds 2005, 4(3): $160-170$.

17. Bus SA: Priorities in offloading the diabetic foot. Diabetes Metab Res Rev 2012, 28 Suppl 1:54-59.

18. Ferris L, Sharkey NA, Smith TS, Matthews DK: Influence of extrinsic plantar flexors on forefoot loading during heel rise. Foot Ankle Int 1995, 16(8):464-473.

19. Akashi PM, Sacco IC, Watari R, Hennig E: The effect of diabetic neuropathy and previous foot ulceration in EMG and ground reaction forces during gait. In: Clin Biomech (Bristol, Avon). vol. 23, 2008/01/08 edn; 2008: 584-592.

20. Kwon OY, Minor SD, Maluf KS, Mueller MJ: Comparison of muscle activity during walking in subjects with and without diabetic neuropathy. Gait Posture 2003, 18(1):105-113.

21. Warren GL, Maher RM, Higbie EJ: Temporal patterns of plantar pressures and lower-leg muscle activity during walking: effect of speed. Gait Posture 2004, 19(1):91-100.

22. Akashi PM, Sacco IC, Watari R, Hennig E: The effect of diabetic neuropathy and previous foot ulceration in EMG and ground reaction forces during gait. Clin Biomech (Bristol, Avon) 2008, 23(5):584-592.

23. Gomes AA, Onodera AN, Otuzi ME, Pripas D, Mezzarane RA, Sacco IC: Electromyography and kinematic changes of gait cycle at different cadences in diabetic neuropathic individuals. Muscle Nerve 2011, 44(2):258-268.

24. Savelberg HH, Ilgin D, Angin S, Willems PJ, Schaper NC, Meijer K: Prolonged activity of knee extensors and dorsal flexors is associated with adaptations in gait in diabetes and diabetic polyneuropathy. Clin Biomech (Bristol, Avon) 2010, 25(5):468-475.

25. Abboud RJ, Rowley DI, Newton RW: Lower limb muscle dysfunction may contribute to foot ulceration in diabetic patients. In: Clin Biomech (Bristol, Avon). vol. 15, 1999/12/11 edn; 2000: 37-45.

26. Sacco IC, Amadio AC: A study of biomechanical parameters in gait analysis and sensitive cronaxie of diabetic neuropathic patients. Clin Biomech (Bristol, Avon) 2000, 15(3):196-202.

27. Uccioli L, Caselli A, Giacomozzi C, Macellari V, Giurato L, Lardieri L, Menzinger G: Pattern of abnormal tangential forces in the diabetic neuropathic foot. Clin Biomech (Bristol, Avon) 2001, 16(5):446-454.

28. Shaw JE, van Schie $\mathrm{CH}$, Carrington $\mathrm{AL}$, Abbott $\mathrm{CA}$, Boulton $\mathrm{AJ}$ : An analysis of dynamic forces transmitted through the foot in diabetic neuropathy. Diabetes Care 1998, 21(11):1955-1959.

29. Yavuzer G, Yetkin I, Toruner FB, Koca N, Bolukbasi N: Gait deviations of patients with diabetes mellitus: looking beyond peripheral neuropathy. Eura Medicophys 2006, 42(2):127-133.

30. Katoulis EC, Ebdon-Parry M, Lanshammar H, Vileikyte L, Kulkarni J, Boulton AJ: Gait abnormalities in diabetic neuropathy. Diabetes Care 1997, 20(12):1904-1907. 
31. Mueller MJ, Minor SD, Sahrmann SA, Schaaf JA, Strube MJ: Differences in the gait characteristics of patients with diabetes and peripheral neuropathy compared with age-matched controls. Phys Ther 1994, 74(4):299-308; discussion 309-213.

32. Savelberg HH, Schaper NC, Willems PJ, de Lange TL, Meijer K: Redistribution of joint moments is associated with changed plantar pressure in diabetic polyneuropathy. BMC Musculoskelet Disord 2009, $10: 16$.

33. Savelberg HH, Schaper NC, Meijer K: The vertical component of the ground reaction force does not reflect horizontal braking or acceleration per se. Clin Biomech (Bristol, Avon) 2009, 24(6):527-528.

34. Giacomozzi C, Caselli A, Macellari V, Giurato L, Lardieri L, Uccioli L: Walking strategy in diabetic patients with peripheral neuropathy. Diabetes Care 2002, 25(8):1451-1457.

35. Vela SA, Lavery LA, Armstrong DG, Anaim AA: The effect of increased weight on peak pressures: implications for obesity and diabetic foot pathology. J Foot Ankle Surg 1998, 37(5):416-420; discussion 448-419.

36. Hills AP, Hennig EM, McDonald M, Bar-Or O: Plantar pressure differences between obese and nonobese adults: a biomechanical analysis. Int J Obes Relat Metab Disord 2001, 25(11):1674-1679.

37. Monteiro M, Gabriel R, Aranha J, Neves e Castro M, Sousa M, Moreira M: Influence of obesity and sarcopenic obesity on plantar pressure of postmenopausal women. Clin Biomech (Bristol, Avon) 2010, 25(5):461-467.

38. Birtane $\mathrm{M}$, Tuna $\mathrm{H}$ : The evaluation of plantar pressure distribution in obese and non-obese adults. Clin Biomech (Bristol, Avon) 2004, 19(10):1055-1059.

39. Monteiro MA, Gabriel RC, Sousa MF, Castro MN, Moreira MH: Temporal parameters of the foot roll-over during walking: influence of obesity and sarcopenic obesity on postmenopausal women. Maturitas 2010, 67(2):178-185.

40. Martinez-Nova A, Pascual Huerta J, Sanchez-Rodriguez R: Cadence, age, and weight as determinants of forefoot plantar pressures using the Biofoot in-shoe system. J Am Podiatr Med Assoc 2008, 98(4):302-310.

41. Wrobel JS, Birkmeyer NJ, Dercoli JL, Connolly JE: Do clinical examination variables predict high plantar pressures in the diabetic foot? J Am Podiatr Med Assoc 2003, 93(5):367-372.

42. Morag E, Cavanagh PR: Structural and functional predictors of regional peak pressures under the foot during walking. J Biomech 1999, 32(4):359-370.

43. Menz HB, Morris ME: Clinical determinants of plantar forces and pressures during walking in older people. Gait Posture 2006, 24(2):229-236.

44. Cavanagh PR, Sims DS, Jr., Sanders LJ: Body mass is a poor predictor of peak plantar pressure in diabetic men. Diabetes Care 1991, 14(8):750-755.

45. Payne C, Turner D, Miller K: Determinants of plantar pressures in the diabetic foot. $J$ Diabetes Complications 2002, 16(4):277-283.

46. Winter DA: The biomechanics and motor control of human gait : normal, elderly and pathological, 2nd edn. Waterloo, Ont.: Waterloo Biomechanics; 1991.

47. Resnick HE, Stansberry KB, Harris TB, Tirivedi M, Smith K, Morgan P, Vinik AI: Diabetes, peripheral neuropathy, and old age disability. Muscle Nerve 2002, 25(1):43-50.

48. Sawacha Z, Gabriella G, Cristoferi G, Guiotto A, Avogaro A, Cobelli C: Diabetic gait and posture abnormalities: a biomechanical investigation through three dimensional gait analysis. Clin Biomech (Bristol, Avon) 2009, 24(9):722-728.

49. Petrofsky J, Lee S, Bweir S: Gait characteristics in people with type 2 diabetes mellitus. Eur J Appl Physiol 2005, 93(5-6):640-647.

50. Resnick HE, Vinik AI, Schwartz AV, Leveille SG, Brancati FL, Balfour J, Guralnik JM: Independent effects of peripheral nerve dysfunction on lower-extremity physical function in old age: the Women's Health and Aging Study. Diabetes Care 2000, 23(11):1642-1647.

51. Segal A, Rohr E, Orendurff M, Shofer J, O'Brien M, Sangeorzan B: The effect of walking speed on peak plantar pressure. Foot Ankle Int 2004, 25(12):926-933.

52. Burnfield JM, Few CD, Mohamed OS, Perry J: The influence of walking speed and footwear on plantar pressures in older adults. Clin Biomech (Bristol, Avon) 2004, 19(1):78-84.

53. Pataky TC, Caravaggi P, Savage R, Parker D, Goulermas JY, Sellers WI, Crompton RH: New insights into the plantar pressure correlates of walking speed using pedobarographic statistical parametric mapping (pSPM). J Biomech 2008, 41(9):1987-1994.

54. Taylor AJ, Menz HB, Keenan AM: Effects of experimentally induced plantar insensitivity on forces and pressures under the foot during normal walking. Gait Posture 2004, 20(3):232-237.

55. Allet L, H IJ, Meijer K, Willems P, Savelberg H: The influence of stride-length on plantar foot-pressures and joint moments. In: Gait Posture. vol. 34, 2011/06/28 edn; 2011: 300-306.

56. Zhu H, Wertsch JJ, Harris GF, Alba HM: Walking cadence effect on plantar pressures. Arch Phys Med Rehabil 1995, 76(11):1000-1005.

57. Drerup B, Szczepaniak A, Wetz HH: Plantar pressure reduction in step-to gait: a biomechanical investigation and clinical feasibility study. Clin Biomech (Bristol, Avon) 2008, 23(8):1073-1079.

58. Zhu HS, Wertsch JJ, Harris GF, Loftsgaarden JD, Price MB: Foot pressure distribution during walking and shuffling. Arch Phys Med Rehabil 1991, 72(6):390-397.

59. Mueller MJ, Sinacore DR, Hoogstrate S, Daly L: Hip and ankle walking strategies: effect on peak plantar pressures and implications for neuropathic ulceration. Arch Phys Med Rehabil 1994, 75(11):1196-1200.

60. York RM, Perell-Gerson KL, Barr M, Durham J, Roper JM: Motor learning of a gait pattern to reduce forefoot plantar pressures in individuals with diabetic peripheral neuropathy. $P M R$ 2009, 1(5):434441. 
61. Brown HE, Mueller MJ: A "step-to" gait decreases pressures on the forefoot. J Orthop Sports Phys Ther 1998, 28(3):139-145.

62. Perez-Soriano P, Llana-Belloch S, Martinez-Nova A, Morey-Klapsing G, Encarnacion-Martinez A: Nordic walking practice might improve plantar pressure distribution. Res Q Exerc Sport 2011, 82(4):593-599.

63. van Sloten TT, Savelberg HH, Duimel-Peeters IG, Meijer K, Henry RM, Stehouwer CD, Schaper NC: Peripheral neuropathy, decreased muscle strength and obesity are strongly associated with walking in persons with type 2 diabetes without manifest mobility limitations. Diabetes Res Clin Pract 2011, 91(1):32-39.

64. Monteiro MA, Gabriel RE, Neves ECM, Sousa MF, Abrantes JM, Moreira MH: Exercise effects in plantar pressure of postmenopausal women. Menopause 2010,17(5):1017-1025.

65. Monteiro MA, Gabriel RE, Sousa MF, Abrantes JM, Moreira MH: Impact of a 12-month exercise program on the temporal parameters of the foot rollover during walking in postmenopausal women. Menopause 2011, 18(7):771-777.

66. Stolwijk NM, Duysens J, Louwerens JW, Keijsers NL: Plantar pressure changes after long-distance walking. Med Sci Sports Exerc 2010, 42(12):2264-2272.

67. Bisiaux M, Moretto P: The effects of fatigue on plantar pressure distribution in walking. Gait Posture 2008, 28(4):693-698. 
Chapter 2 


\section{Chapter 3}

\section{Calculation of plantar pressure-time integral, an alternative approach}

Tom Melai, T. Herman IJzerman, Nicolaas C. Schaper, Ton L.H. de Lange, Paul J.B. Willems, Kenneth Meijer, Aloysius G Lieverse, Hans H.C.M. Savelberg.

Gait and Posture, 2011. 34(3): p. 379-83. 


\section{Abstract}

In plantar pressure measurement, the pressure-time integral is used as an additional variable next to peak pressure to assess plantar loading. However, this variable shows a high concordance with peak pressure. Many researchers and clinicians use Novel software that calculates this variable as the summation of the products of peak pressure and duration per frame, which is not an integral of pressure over time. Therefore, an alternative calculation method is introduced. The aim of this study was to explore the relevance of this alternative method in different populations.

Plantar pressure variables were measured in 76 people with diabetic polyneuropathy, 33 diabetic controls without polyneuropathy and 19 healthy subjects. Peak pressure and pressure-time integral were obtained using Novel software. The quotient of the genuine force-time integral over contact area was obtained as the alternative pressure-time integral calculation.

The new alternative method correlated less with peak pressure than the pressure-time integral as calculated by Novel. The two methods differed significantly and these differences varied between the foot sole areas and between groups. The largest differences were found under the metatarsal heads in the group with diabetic polyneuropathy.

From a theoretical perspective, the alternative approach provides a more valid calculation of the pressure-time integral. In addition, this study showed that the alternative calculation is of added value, along peak pressure calculation, to interpret adapted plantar pressures patterns in particular in patients at risk for foot ulceration. 


\section{Introduction}

People with diabetic polyneuropathy (DPN) have a higher risk to develop plantar ulceration, especially under the metatarsal heads [1]. This higher risk has partially been attributed to high mechanical stresses $[2,3]$. It is therefore important to measure the mechanical loading of the plantar area of the foot to identify the patients at risk for future foot ulceration and to implement strategies to reduce this abnormal loading of the foot. Peak Pressure (PP) is the most commonly used plantar pressure variable to express foot loading. This value represents the maximal load in an area under the foot during one step. Different authors described the association of PP and subsequent tissue damage [4,5]. PPs that exceed the threshold of $400 \mathrm{~N} / \mathrm{cm}^{2}$ can cause direct trauma of the skin [6], but pressures above 70 or $150 \mathrm{~N} / \mathrm{cm}^{2}$ can cause trauma as well $[5,6]$. A repetitive exposure to these sub threshold pressures could inhibit regeneration processes and the cumulative effect could eventually lead to tissue failure $[7,8]$.

Another variable that is increasingly used in evaluating plantar loading is the Pressure-time Integral (PTI). This variable describes the cumulative effect of pressure over time in a certain area of the foot, and thus provides a value for the total load exposure of a foot sole area during one step [1]. Because, as stated above, cumulative exposure could lead to tissue damage, this variable could be a risk assessor for skin trauma as well. Nevertheless, according to Waaijman and Bus [9], there is a high coherence of PP and PTI. These authors found high correlations between PP and PTI using a Novel Pedar-X system for different footwear conditions [9]. Consequently, they suggested that reporting both variables might be of limited value. However, when using Novel software the PTI (PTI_N) is calculated as the summation of the products of PP per frame and the duration of that frame.

$$
\text { PTI_N }=\sum \mathrm{PP}_{\mathrm{i}}{ }^{*} \Delta \mathrm{t} \quad \text { (1) where } \mathrm{PP}_{\mathrm{i}} \text { is the peak pressure in the } \mathrm{i} \text {-th frame and } \Delta \mathrm{t} \text { is }
$$
the duration of that frame.

As in this approach the PTI will be a summation of only the peak pressures per frame, it does not represent an exact integration of pressure over time. Consequently, it does not include information about the sub maximal pressures under one plantar area during one frame and does not give adequate insight in the summated loading of a particular plantar area. An alternative variable within the Novel software, that does include the entire load an area is exposed to, is the Force-time Integral (FTI). This variable is a genuine integral of force over time in a particular foot sole area:

$$
\mathrm{FTI}=\int \mathrm{F}^{*} \Delta \mathrm{t} \quad \text { (2) where, } \int \mathrm{F}^{*} \Delta \mathrm{t} \text { is the integral of force over time }
$$

However, the FTI does not account for the size of an area it is applied to. If the FTI in a region is higher, than this does not automatically mean that the skin tissue of this area is at risk of overloading. The smaller an area a force is applied to, the greater the effect. Therefore it is hard to relate this variable to plantar tissue damage. If the FTI of a certain region is divided by the contact area of that region, it will provide a measure for the cumulative load an area is exposed to, that does account for the size of that area. Because it is a quotient of FTI over contact area, it will 
provide the mean cumulative load per squared centimeter, and consequently a more meaningful calculation of the pressure-time integral (PTI_F):

$$
\text { PTI_F }=\text { FTI/ A }
$$

(3) where, FTI is obtained from equation 2, and A is the contact area of the particular foot sole area.

In this study, we explored whether the theoretically more valid calculation of the pressure-time integral (PTI_F) is of added value in understanding and interpreting plantar pressure patterns. We hypothesized that PTI_F will result in lower values for the summated pressures than PTI_N. In addition, we expect that the differences between PTI_N and PTI_F will be larger in an area where there is more variation of pressure (cf. figure $1 \mathrm{a}$ and $1 \mathrm{~b}$ ).

To test the clinical relevance of PTI_F, we sought to test these hypotheses in different populations: with and without diabetes and with and without polyneuropathy, as this is a population that is at higher risk for abnormal foot loading.

\section{Methods}

\section{Subjects}

Three groups of subjects were recruited (table 1): 19 healthy elderly subjects (HE, mean age 68.1 (SD 5.2)) were recruited by means of advertisement or participation in previous research. Subjects with type 2 diabetes were recruited from the diabetes clinics of the Maastricht University Medical Center, Maxima Medical Center Eindhoven and Veldhoven, St. Anna Hospital Geldrop and Maasland Hospital Sittard. All these patients underwent a standardized neurological examination which included reflexes, vital and gnostic sensibility and lower extremity muscle strength [10]. Based on a validated scoring system the diagnosis of diabetic polyneuropathy (DPN) was made in 76 patients (DPN, mean age 66.0 (SD 7.2)). A group of 33 diabetic controls did not have clinical signs of polyneuropathy (DC, mean age 62.8 (SD 7.1)). All subjects were above 50 years of age and were able to walk 6 minutes without walking aids. Subjects were excluded if diagnosed with severe cardiac disease, renal dysfunction (creatinin $>180 \mu \mathrm{mol} / \mathrm{I}$ ), intermittent claudication, neurological
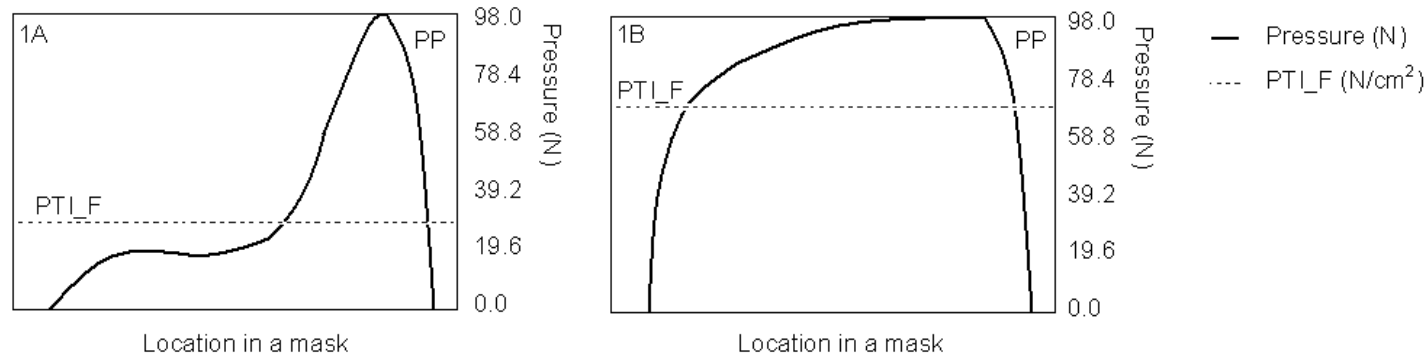

Figure 1. ( $A$ and B) Two-dimensional representation of three-dimensional time samples of pressure measurement. The horizontal axes of these graphs resemble the fore-aft dimension of a 2D masking area. The vertical axis shows the fluctuation in loading within this area. Peak pressure (PP) and duration of the sample are similar for both time samples. Calculation of the pressure-time integral, as provided by the Novel software (PTI N) will therefore result in identical outcomes for both time samples, while the cumulative load, the area under the curve, of the right time sample (1B) is much higher than the left time sample (1A). As shown here, the alternative calculation method for cumulative loading (PTI_F) is sensitive for variations of pressure within one time sample. 
disorders other than DPN, rheumatoid arthritis, severe osteoarthritis, foot deformities or amputations, or foot ulceration. All participants signed an informed consent, the protocol was approved by the medical ethical committees of the hospitals involved and all experimental procedures complied with the principles laid down in the Declaration of Helsinki.

\section{Protocol}

Subjects were asked to walk barefooted over a wooden walkway $(7 \mathrm{~m})$ with an imbedded pressureplatform (EMED-x $\left(100 \mathrm{~Hz}, 4\right.$ sensors $/ \mathrm{cm}^{2}$, range $\left.0-127 \mathrm{~N} / \mathrm{cm}^{2}\right)$ or EMED-at $\left(50 \mathrm{~Hz}, 2\right.$ sensors $/ \mathrm{cm}^{2}$, range 0-120 N/cm ${ }^{2}$ ), Novel Inc., Munich, Germany).

Subjects were allowed to familiarize themselves with the test settings. Walking speed was standardized by means of infra-red detection portals (range: 1.1 to $1.3 \mathrm{~m} / \mathrm{s}$ ). Pressure data of the right foot was collected from 5 trials.

\section{Analysis}

Plantar pressure data were masked in Novel Database Medical (13.3.42, Germany 2007) using the Novel 10 mask division (area $1=$ heel, area $2=$ mid foot, areas $3-7=$ metatarsal region, area $8=$ hallux, areas 9 and $10=$ smaller toes). Trials were excluded if there were inconsistencies in the automatic masking procedure, due to for example dragging of the hallux over the pressureplatform.

An ASCII output was generated per trial of which PP, PTI_N, FTI and contact area were obtained. Using Matlab (R2007b, USA 2007), PTI_F was calculated by dividing FTI output by contact area for each mask. Averages were determined per subject based on a minimum of three correct trials.

\section{Statistical Analysis}

All data were analyzed using SPSS 15.0 for Windows. Loglineair (base 10) transformation was used for not normally distributed data (based on Shapiro- Wilk). Correlations between the variables PTI_F and PP, and PTI_N and PP were determined using Spearman's correlation coefficient. A repeated measures multivariate analysis of variance was performed with plantar area and calculation method as within factors, and subject group as between factor to examine the differences between the two calculation methods. These were considered significant if $p \leq 0.05$. Differences between groups (HE, DC or DPN) were analyzed using a repeated measures analysis of variance (post hoc: Games Howel) with a Bonferroni correction for the number of tests performed (10). Differences were therefore considered significant if $p \leq 0.005$.

\section{Table 1. Subject characteristics}

\begin{tabular}{|c|c|c|c|c|}
\hline & $\mathrm{HE}(n=19)$ & DC $(n=33)$ & DPN $(n=76)$ & $P$ \\
\hline Age (years) & $68.1(5.2)$ & $62.8(7.1)$ & $66.0(7.2)$ & 0.075 \\
\hline Length (m) & $1.73(0.07)$ & $1.69(0.10)$ & $1.74(0.08)$ & 0.029 \\
\hline Weight (kg) & $72.6(8.5)$ & $88.4(16.0)$ & $94.4(20.7)$ & 0.000 \\
\hline
\end{tabular}

Data are presented for subjects included for statistical analysis as mean (standard deviation) and $p$-value per group based on Kruskal Wallis testing. Differences are considered significant if $p \leq 0.05$. Abbreviations: HE: Healthy Elderly, DC: Diabetic Controls, DPN: Diabetic Polyneuropathy. 
Table 2. Correlation of plantar pressure variables

\begin{tabular}{lrrrrrrrrrr}
\hline Plantar area & Heel & Mid & MT1 & MT2 & MT3 & MT4 & MT5 & Hallux & Dig 2 & Dig 3-5 \\
\hline PTI_N and PP & 0.64 & 0.69 & 0.83 & 0.85 & 0.76 & 0.74 & 0.90 & 0.83 & 0.88 & 0.83 \\
PTI_F and PP & 0.36 & 0.64 & 0.56 & 0.37 & 0.31 & 0.41 & 0.69 & 0.52 & 0.72 & 0.59 \\
\hline
\end{tabular}

Data are presented as mean $R^{2}$, all values were significant at 0.01 level. Abbreviations: PP: Peak Pressure, PTI_N: Pressure-time Integral based on Novel software, PTI_F: Pressure-time Integral based on Force-time Integral, MT: Metatarsal area, Dig: Digitorum.

\section{Results}

PTI_N and PP were highly correlated for all foot sole areas: $\mathrm{R}^{2}$ ranged from 0.64 in the heel to 0.90 under the fifth metatarsal head (table 2). PTI_F correlated less with PP, especially in the regions under the heel and the second to fourth metatarsal heads, where only 31 to $41 \%$ of the variance of PP could be explained by PTI_F (table 2).

Statistical analysis showed that the two methods differed significantly $(p<0.001)$ and that these differences varied in the different areas under the foot (calculation method * plantar area interaction, $p<0.001$; table 3). The largest differences between these two variables were observed in the area under the medial metatarsal heads and the hallux. The smallest differences occurred under the smaller toes and the mid foot (figure 2).

Not only did the different calculation methods result in different PTIs per foot region in general, but in significant group differences as well (calculation method*group interaction, $p=$ 0.015, table 3, figure 2). In addition differences tended to vary over groups between areas (method $*$ plantar area $*$ group interaction $p=0.063$ ). Post hoc analysis showed that the groups differed significantly in the areas under the first and second metatarsal head $(p=0.005,0.003$ respectively, table 3 , figure 2 ). In the DPN group differences between calculation methods were significantly larger than in both other groups under the first (DPN - HE: $p=0.001$, DPN - DC: $p=$ 0.005 ) and second metatarsal area (DPN - HE: $p=0.003$, DPN - DC: $p=0.012$ ). In the other foot sole areas the differences between calculation methods did not vary over the groups.

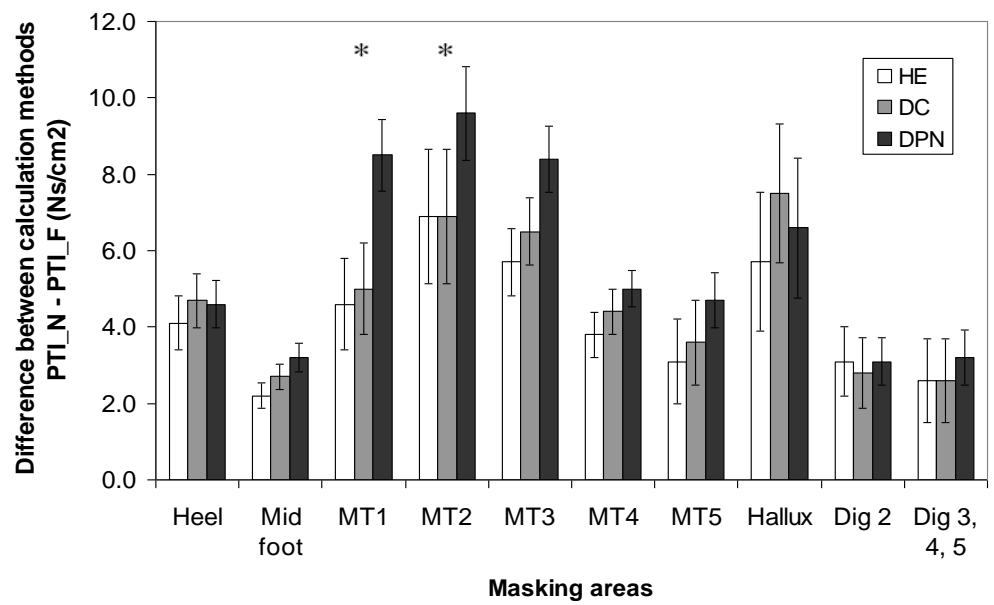

Figure 2. Mean differences and confidence intervals between calculation methods of PTI (PTI_N - PTI_F), for 10 different plantar areas in 3 different populations. Abbreviations: PP: Peak Pressure, PTI_N: Pressure-time Integral based on Novel software, PTI_F: Pressure-time Integral based on Force-time Integral, MT: Metatarsal area, Dig : Digitorum, HE: Integral Healthy Elderly, DC: Diabetic Controls, DPN: Diabetic Polyneuropathy. Significant differences $(P<0.005)$ between groups are indicated by *. 
Table 3. Averages of plantar pressure variables over the different groups per foot sole area

\begin{tabular}{|c|c|c|c|c|c|c|c|c|c|c|}
\hline & & \multicolumn{3}{|c|}{$\operatorname{HE}(n=19)$} & \multicolumn{3}{|c|}{$D C(n=33)$} & \multicolumn{3}{|c|}{ DPN $(n=76)$} \\
\hline & & $\begin{array}{r}\text { PTI_N } \\
\left(\mathrm{Ns} / \mathrm{cm}^{2}\right)\end{array}$ & $\begin{array}{r}\text { PTI F } \\
\left(\mathrm{Ns} / \mathrm{cm}^{2}\right)\end{array}$ & $\begin{array}{r}\mathrm{PP} \\
\left(\mathrm{N} / \mathrm{cm}^{2}\right)\end{array}$ & $\begin{array}{r}\text { PTI_N } \\
\left(\mathrm{Ns} / \mathrm{cm}^{2}\right)\end{array}$ & $\begin{array}{r}\text { PTI F } \\
\left(\mathrm{Ns} / \mathrm{cm}^{2}\right)\end{array}$ & $\begin{array}{r}\mathrm{PP} \\
\left(\mathrm{N} / \mathrm{cm}^{2}\right)\end{array}$ & $\begin{array}{r}\text { PTI_N } \\
\left(\mathrm{Ns} / \mathrm{cm}^{2}\right)\end{array}$ & $\begin{array}{r}\text { PTI F } \\
\left(\mathrm{Ns} / \mathrm{cm}^{2}\right)\end{array}$ & $\begin{array}{r}P P \\
\left(\mathrm{~N} / \mathrm{cm}^{2}\right)\end{array}$ \\
\hline \multirow[t]{3}{*}{ Heel } & mean & 7.2 & 3.1 & 35.9 & 8.1 & 3.5 & 41.9 & 8.0 & 3.4 & 42.5 \\
\hline & $S D$ & $(2.0)$ & $(0.7)$ & (9.3) & $(2.3)$ & $(0.7)$ & (10.9) & $(2.5)$ & $(0.9)$ & $(11.8)$ \\
\hline & $C V$ & 0.28 & $0.23 *$ & 0.26 & 0.28 & $0.20 *$ & 0.26 & 0.31 & $0.26 *$ & 0.28 \\
\hline \multirow[t]{3}{*}{ Mid foot } & mean & 3.1 & 1.0 & 11.8 & 4.7 & 1.5 & 16.5 & 3.9 & 1.2 & 15.0 \\
\hline & $S D$ & $(0.9)$ & $(0.1)$ & $(2.4)$ & $(1.5)$ & $(0.6)$ & $(6.0)$ & $(1.7)$ & $(0.6)$ & $(5.2)$ \\
\hline & $C V$ & 0.29 & $0.10 *$ & 0.20 & $0.32 *$ & 0.40 & 0.36 & 0.44 & 0.50 & $0.35^{*}$ \\
\hline \multirow[t]{3}{*}{ MT1 } & mean & 7.8 & 3.2 & 27.3 & 8.9 & 3.9 & 31.5 & 13.2 & 4.7 & 44.9 \\
\hline & $S D$ & $(3.3)$ & $(1.0)$ & $(9.2)$ & $(3.4)$ & $(1.0)$ & $(12.1)$ & $(8.4)$ & $(2.0)$ & $(24.4)$ \\
\hline & $C V$ & 0.42 & $0.31 *$ & 0.34 & 0.38 & $0.26 *$ & 0.38 & 0.64 & $0.43^{*}$ & 0.54 \\
\hline \multirow[t]{3}{*}{ MT2 } & mean & 12.3 & 5.4 & 47.5 & 12.9 & 6.0 & 51.0 & 16.1 & 6.5 & 62.4 \\
\hline & $S D$ & $(4.5)$ & $(1.3)$ & $(22.1)$ & $(4.1)$ & $(1.2)$ & (20.1) & $(5.8)$ & $(1.7)$ & $(26.2)$ \\
\hline & $\mathrm{CV}$ & 0.37 & $0.24 *$ & 0.47 & 0.32 & $0.20 *$ & 0.39 & 0.36 & $0.26 *$ & 0.42 \\
\hline \multirow[t]{3}{*}{ MT3 } & mean & 10.6 & 5.0 & 36.4 & 12.3 & 5.8 & 44.8 & 14.3 & 5.9 & 50.1 \\
\hline & $S D$ & $(2.4)$ & $(1.0)$ & $(7.5)$ & $(3.4)$ & $(1.4)$ & (13.3) & $(5.1)$ & $(1.3)$ & $(19.8)$ \\
\hline & $C V$ & 0.23 & $0.20 *$ & 0.21 & 0.28 & $0.24 *$ & 0.30 & 0.36 & $0.22 *$ & 0.40 \\
\hline \multirow[t]{3}{*}{ MT4 } & mean & 7.6 & 3.8 & 23.6 & 9.2 & 4.7 & 29.6 & 9.4 & 4.4 & 30.3 \\
\hline & $S D$ & $(1.9)$ & $(1.0)$ & $(5.2)$ & $(2.3)$ & $(1.3)$ & $(7.0)$ & $(2.9)$ & $(1.2)$ & $(11.3)$ \\
\hline & $C V$ & 0.25 & 0.26 & $0.22 *$ & 0.25 & 0.28 & $0.24 *$ & 0.31 & $0.27 *$ & 0.37 \\
\hline \multirow[t]{3}{*}{ MT5 } & mean & 5.8 & 2.7 & 19.2 & 7.0 & 3.3 & 24.4 & 8.0 & 3.2 & 28.9 \\
\hline & $S D$ & $(3.2)$ & $(1.1)$ & (11.9) & $(2.9)$ & $(1.2)$ & (14.1) & $(5.5)$ & $(1.3)$ & $(20.4)$ \\
\hline & $C V$ & 0.55 & $0.41 *$ & 0.62 & 0.41 & $0.36 *$ & 0.58 & 0.69 & $0.41 *$ & 0.71 \\
\hline \multirow[t]{3}{*}{ Hallux } & mean & 8.1 & 2.5 & 35.5 & 10.2 & 2.7 & 51.4 & 9.3 & 2.6 & 46.3 \\
\hline & $S D$ & $(4.5)$ & $(1.0)$ & (14.9) & $(6.0)$ & $(1.1)$ & $(28.6)$ & $(5.3)$ & $(1.1)$ & $(24.3)$ \\
\hline & $C V$ & 0.56 & $0.40 *$ & 0.42 & 0.59 & $0.41 *$ & 0.56 & 0.57 & $0.42 *$ & 0.52 \\
\hline \multirow[t]{3}{*}{ Dig 2} & mean & 4.9 & 1.8 & 21.5 & 4.3 & 1.5 & 21.4 & 4.7 & 1.6 & 22.3 \\
\hline & $S D$ & $(2.6)$ & $(0.9)$ & $(9.7)$ & $(2.3)$ & $(0.7)$ & (10.1) & $(2.8)$ & $(0.8)$ & $(12.8)$ \\
\hline & $C V$ & 0.53 & 0.50 & $0.45^{*}$ & 0.53 & $0.47 *$ & $0.47 *$ & 0.60 & $0.50 *$ & 0.57 \\
\hline \multirow{3}{*}{$\begin{array}{l}\text { Dig } 3, \\
4,5\end{array}$} & mean & 4.5 & 1.3 & 16.2 & 3.6 & 1.0 & 15.4 & 3.6 & 1.0 & 15.3 \\
\hline & $S D$ & $(2.8)$ & $(0.7)$ & $(7.2)$ & $(2.6)$ & $(0.6)$ & $(8.1)$ & $(2.0)$ & $(0.5)$ & (7.1) \\
\hline & $\mathrm{CV}$ & 0.62 & 0.54 & $0.44 *$ & 0.72 & 0.60 & $0.53 *$ & 0.56 & 0.50 & $0.46 *$ \\
\hline
\end{tabular}

Data are presented as means, standard deviations (SD) and Coefficient of Variation (CV). The lowest CV within one group is marked with *. Abbreviations: PP: Peak Pressure, PTI_N: Pressure-time Integral based on Novel software, PTI_F: Pressure-time Integral based on Force-time Integral, MT: Metatarsal area, Dig: Digitorum, HE: Integral Healthy Elderly, DC: Diabetic Controls, DPN: Diabetic Polyneuropathy.

\section{Discussion}

Our findings indicate that PTI_N largely reflects PP, given the strong correlation between PTI_N and PP. Moreover, the PTI_N is probably not a physically meaningful measure of cumulative load. In contrast, the alternative calculation method for cumulative loading (PTI_F), as described in this study, seems to provide additional information on top of PP when loading characteristics of the foot are evaluated in different populations. The high correlations obtained by barefoot measurement of PTI_N and PP supported the results of Waaijman and Bus gained by in shoe measurement [9]. The high correlations between these variables are not surprisingly, because PTI_N is calculated from PP. In contrast, correlation analysis of PTI_F and PP showed that only a relatively small part of the variance of PP could be explained by PTI_F. This indicates that the fluctuation of pressures measured by the PTI_F provides different information on foot loading in addition to PP.

The question rises whether this alternative approach provides relevant information for clinical practice or scientific analysis. This seems to be the case, since the correlations of PTI_F to 
PP show more variation over foot sole regions in contrast to the correlations of PTI_N to PP (table 2). Differences in instantaneous variations in pressure seem to underlie this low correlation between PP and PTI_F. The PP will only be affected by variations of the highest overall pressure. And although PTI_N is sensitive to variations of the highest pressure in the different frames, it will not be affected by variations of sub maximal pressures. In contrast, the PTI_F seems to be sensitive enough to measure these changes in sub maximal loading. This is shown especially in the areas under the heel and the metatarsal areas and suggests that PTI_F contains information on mechanical loading of the foot sole that is not provided by PP or the PTI_N.

Moreover, the multivariate analysis of variance confirmed the differences between the two calculation methods and foot sole regions, indicating additional value to study foot loading. Even more, the PTI_F seems to have a clinical value as well. Although the interaction effect was not significant, the data showed that there was a tendency for differences between groups $(p=0.063)$. The group with DPN seemed to differ from the other two groups in two of the most relevant areas for this population, the areas under the medial metatarsal heads. Analysis of the data in table 3 illustrates that in this region PTI_F increases less over the groups (from HE via DC to DPN) than PP and PTI_N. Therefore, the pressure patterns for these metatarsal areas are characterized by an increased PP and not so much by a high cumulative pressure, if analyzed by PTI_F. If PTI_N would have been adopted as the measure of cumulative loading one would erroneously conclude that not only the PP, but also the cumulative stress in these foot sole regions was higher in the DPN participants.

Our analyses suggest that PP and PTI_N do not include all the information there is on foot loading, especially in the area under the metatarsal heads. As this area is prone to abnormal loading during ambulation and subsequent ulceration in patients with diabetic neuropathy [1], the absolute load and its pattern in these areas need to be estimated as correctly as possible, in order to prevent future ulceration.

When using the PTI_F it is important to regard areas of limited size. If the cumulative loading is increased in a certain area of the foot, it will become visible when the mask includes that area. However when calculating the PTI_F of the whole foot the effect of this increased loading will be leveled out. The PTI_N is also affected by the size of the mask. However, this does not only affect the response validity of PTI_N, it will affect the accuracy as well. A large region will lead to more variation of pressure within one frame. And because PTI_N is only affected by the peak pressures, the validity of this variable will decrease when the size of the mask increases. For these reasons, regardless of the calculation method, the PTI will only be meaningful when masks are of limited size.

A limitation of the PTI_F calculation method is that the total contact area of one region is used, while the instantaneous extent of contact area may vary over time. However, this is presumably only a theoretical limitation, because for the major part of the contact time of a foot sole area, most sensors of a specific area are loaded.

A limitation of the study is that outcome was incidentally affected by the measuring range of the platforms used. Three subjects (all DPN) exceeded the maximal measuring range, which was visible by clipping of the pressure graph. As a result of such a cut off, not only PP was affected, but cumulative loading as well. When pressure is leveled, the differences between the two variables will 
be less, which will result in convergence. It is therefore expected that if the platform was able to measure higher pressures, the differences between PTI_F and PTI_N would even be larger.

Although this study shows that cumulative loading, measured by means of PTI_F, may differ from the PPs in the separate areas under the foot, it does not provide any information on the clinical relationship with plantar tissue damage. Therefore, future research should aim at clarifying the clinical relevance of cumulative loading, using the quotient of FTI and contact area.

\section{Conclusion}

This study compared an alternative approach to calculate PTI in contrast to the PTI provided by Novel software. It can be concluded that the latter probably does not correctly measure cumulative loading because it only regards peak pressures within the separate frames. The alternative approach seems to provide a more valid PTI, because it includes not only the peak pressures, but the sub maximal pressures as well. Even more this variable seems to hold additional information on foot loading.

Comparison of the alternative calculation methods for PTI between different populations and foot sole areas demonstrated that this alternative calculation of PTI is not only more valid than PTI_N, but is of added value to understand plantar pressure patterns between groups as well. 


\section{References}

1. Sauseng S, Kästenbauer T, Sokol G, Irsigler K: Estimation of risk for plantar foot ulceration in diabetic patients with neuropathy. Diabetes Nutr Metab 1999, 12: 189-193.

2. Veves A, Murray $\mathrm{HJ}$, Young MJ, Boulton AJ: The risk of foot ulceration in diabetic patients with high foot pressure: a prospective study. Diabetologia 1992, 35: 660-663.

3. Pham H, Armstrong DG, Harvey C, Harkless LB, Giurini JM, Veves A: Screening techniques to identify people at high risk for diabetic foot ulceration: a prospective multicenter trial. Diabetes Care 2000, 23: 606-611.

4. Boulton AJM, Hardistry CA, Betts RP, Franks CI, Worth RC, Ward JD et al: Dynamic Foot Pressure and Other Studies as Diagnostic and Management Aids in Diabetic Neuropathy. Diabetes Care 1983, 6: 26-33.

5. Armstrong DG, Peters EJG, Athanasiou KA, Lavery LA: Is there a critical level of plantar foot pressure to identify patients at risk for neuropathic foot ulceration? J Foot Ankle Surg 1998, 37: 303-307.

6. Birke JA, Novick A, Hawskins ES, Patout Jr C: A review of Causes of Foot Ulceration in Patients with Diabetes Mellitus. J Prosthet. Orthot 1991, 4: 13-22.

7. Hall OC, Brand PW: The Etiology of the neuropathic plantar ulcer. J Am Podiatry Assoc 1979, 69:173-177

8. Maluf KS, Mueller MJ: Comparison of physical activity and cumulative plantar tissue stress among subjects with and without diabetes mellitus and a history of recurrent plantar ulcers. Clin Biomech 2002, 18: 567575.

9. Waaijman R, Bus S: Association between peak pressure and pressure time integral in footwear studies on the diabetic foot: what is the need to report both variables? Ned Tijdschr Diabetologie 2009, 7: 124-125.

10. Valk GD, Nauta JJP, Strijers RLM, Bertelsman FW: Clinical examination versus neurophysiological examination in the diagnosis of polyneuropathy. Diabet Med 1992, 9: 716-721. 


\section{Chapter 4}

\section{Increased forefoot loading is associated with an increased plantar flexion moment}

Tom Melai, T. Herman IJzerman, Nicolaas C. Schaper, Ton L.H. de Lange, Paul J.B. Willems, Kenneth Meijer, Aloysius G Lieverse, Hans H.C.M. Savelberg.

Human Movement Science, 2013. In press 


\section{Abstract}

The aim of this study was to identify the cascade of effects leading from alterations in force generation around the ankle joint to increased plantar pressures under the forefoot.

Gait analysis, including plantar pressure measurement, was performed at an individually preferred and a standardized, imposed gait velocity in diabetic subjects with polyneuropathy ( $\mathrm{n}=$ $94)$, without polyneuropathy $(n=39)$ and healthy elderly $(n=19)$.

The plantar flexion moment at $40 \%$ of the stance phase was negatively correlated with the displacement rate of center of pressure $(r=-0.749, p<0.001$ at imposed, and $r=-0.693, p<0.001$ at the preferred gait velocity). Displacement rate of center of pressure was strongly correlated with forefoot loading $(r=-0.837, p<0.001$ at imposed, and $r=-0.731, p<0.001$ at the preferred gait velocity).

People with a relative high plantar flexion moment at $40 \%$ of the stance phase, have a faster forward transfer of center of pressure and consequently higher loading of the forefoot. This indicates that interventions aimed at increasing the control of the roll-off of the foot may contribute to a better plantar pressure distribution. 


\section{Introduction}

Plantar foot ulceration is an invalidating co-morbidity of diabetes. Increased plantar pressure during walking is an important risk factor for (recurrent) plantar ulceration [1] and it has been shown that the plantar pressure pattern is altered in patients with diabetes and polyneuropathy (DPN) $[2,3]$. Several authors showed that in this population especially the forefoot is prone to higher pressures and consequently at risk for ulceration [3,4].

Diabetes has been associated with several structural and functional alterations in the foot that can result in higher plantar pressures, such as anatomical deformities (claw toes, charcot neuro-osteoarthropathy), limited joint mobility and skin changes (callus formation) [5-10]. Moreover, it has been suggested that changes in gait biomechanics can lead to higher loading of the foot in DPN patients [11, 12]. Gait in DPN is characterized by longer stance phases, shorter steps and lower gait velocity [11, 13-15]. In this context Van Deursen et al. [16] argued that deficits of the sensory nerve system could lead to alteration of gait in this population. The sensory nerve system provides information on the interaction with the ground and on the tension of the muscles. If the feedback from this system is reduced, it can lead to insecurity of gait or limitation in motor control resulting in abnormal loading of the foot $[13,16,17]$.

In addition to its effect on the sensory system, DPN can also affect the motor nervous system. Abboud et al. found a delayed activation of the tibial anterior muscle [18], while others found prolonged activity of this muscle [19] or premature activation of the calf muscles in DPN [15]. Besides possible changes in muscle activation patterns, DPN affects the force generating ability. Several authors found reduced lower extremity muscle strength in people with diabetes [12, 20, 21]. In addition, Andreassen et al. [22] observed weakness of the ankle plantar and dorsiflexors in particular in DPN patients with severe neuropathy. Several authors found a combination of lower extremity muscle dysfunctioning and increased plantar pressures. They suggested that a decrease in motor control due to muscle weakness or limited feedback might be partly responsible for the observed increased plantar pressures in patients with DPN $[15,18]$. In an earlier study we found higher plantar flexion moments from loading response to mid stance and an increase in plantar loading in this population [11]. We suggested that reduced strength of the lower extremity muscles (caused by DPN) resulted in an impaired ability to brake the forward momentum of the body during the first part of the stance phase. This would have led to a faster roll-off of the foot, with a faster forward transfer of the center of pressure (COP) and consequently causing higher plantar forefoot loading. In the current study we tested the hypothesis that via the aforementioned cascade of relations, adaptations in force generation around the ankle joint result in a forwardly displaced plantar pressure pattern during gait. This hypothesis was tested in people with and without diabetes, and with and without polyneuropathy.

\section{Methods}

\section{Participants}

Three groups of subjects participated, with a total number of 152 (table 1). A group of 19 healthy elderly subjects $(\mathrm{HE}$, mean age of $68(\mathrm{SD}=5)$ years) was recruited by means of advertisement or 
participation in previous research. Subjects with diabetes type 2 were recruited from the diabetes clinics of 3 general and 1 academic hospital in the Netherlands. All participants underwent a standardized Clinical Neurological Examination (CNE) to determine the presence of DPN. The CNE included reflexes, vital and gnostic sensibility and lower extremity muscle strength [23]. Based on this validated scoring system 94 patients $(67(S D=8)$ years) were diagnosed with DPN and 39 diabetic controls did not have clinical signs of polyneuropathy (DC, $62(S D=7)$ years). All subjects were above 50 years of age and were able to walk 6 minutes without walking aids. Subjects were excluded if diagnosed with severe cardiac disease, renal dysfunction (creatinin> $180 \mu \mathrm{mol} / \mathrm{I}$ ), intermittent claudication, neurological disorders other than DPN, rheumatoid arthritis, severe osteoarthritis, major foot deformities or amputations, or prior or current foot ulceration. All participants signed an informed consent; the protocol was approved by the medical ethical committee of Maastricht University Medical Center+.

Table 1. Subject characteristics

\begin{tabular}{|c|c|c|c|c|}
\hline Group & DPN $(n=94)$ & $D C(n=39)$ & $\mathrm{HE}(\mathrm{n}=19)$ & $p$-value \\
\hline Sex (male/ female) & $80 / 14^{a}$ & $20 / 19^{b}$ & $15 / 4$ & 0.001 \\
\hline Age (years) & $66.9(7.5)^{a}$ & $62.4(6.6)^{\mathrm{b}}$ & $67.8(5.30$ & 0.002 \\
\hline Body height (m) & $1.74(0.08)^{\mathrm{a}}$ & $1.68(0.10)$ & $1.73(0.07)$ & 0.002 \\
\hline Body mass (kg) & $92.9(19.5)^{b}$ & $88.0(15.4)^{\mathrm{b}}$ & $68.8(17.1)$ & $<0.001$ \\
\hline BMI $\left(\mathrm{kg} \cdot \mathrm{m}^{2}\right)$ & $30.5(5.6)^{b}$ & $31.1(5.7)^{\mathrm{b}}$ & $23.2(5.6)$ & $<0.001$ \\
\hline CNE score & $12.9(5.2)^{\mathrm{ab}}$ & $2.5(1.6)^{b}$ & $1.4(1.5)$ & $<0.001$ \\
\hline HbA1c (\%) & $7.3(1.1)^{\mathrm{b}}$ & $7.1(1.0)^{\mathrm{b}}$ & $6.0(0.5)$ & $<0.001$ \\
\hline Glucose (mmol/L) & $8.5(2.4)^{b}$ & $8.0(2.2)^{b}$ & $5.6(0.6)$ & $<0.001$ \\
\hline Creatinin $(\mu \mathrm{mol} / \mathrm{L})$ & $98.3(30.0)^{a}$ & $81.2(22.2)$ & $89.0(12.4)$ & 0.003 \\
\hline
\end{tabular}

Data are presented for the subjects included in the study as mean (SD). Abbreviations: DPN: Diabetic polyneuropathy; DC: Diabetic Control; HE: Healthy Elderly; CNE: Clinical Neurological Examination. The pvalues show overall group differences for each variable. ${ }^{a}$ Differs significantly from DC, and ${ }^{b}$ differs significantly from $H E$, based on post hoc analysis.

\section{Experimental set-up}

Subjects were tested in the gait laboratories of two institutes in the southern part of the Netherlands. For gait analysis at the first institute a wooden walkway was available with embedded a pressure-platform (EMED-x, $100 \mathrm{~Hz}, 4$ sensors/cm², range 0-127 N/cm², Novel Inc., Munich, Germany) mounted on top of the force-platform (AMTI LG6_2-1,Advanced Medical Technology Inc, Newton , MA, US). At the second institute a similar set-up was present, with a comparable force and pressure-platform (force-platform: Kistler 9281A SN, Kistler instrumente AG, Winterhur Switzerland; pressure-platform: $50 \mathrm{~Hz}, 2$ sensors $/ \mathrm{cm}^{2}$, range 0-120 N/cm², Novel Inc., Munich, Germany). At both sites a digital high speed camera (1.3 megapixel Pixellink PL-A741, sampling at $100 \mathrm{~Hz}$ ) was used to assess 2D kinematic data in the sagittal plane. Participants were equipped with reflective markers attached to the skin at the middle of the greater trochanter, lateral femoral epicondyle, calcaneus, lateral malleolus and fifth metatarsal of the right leg. Gait velocity was determined by means of the horizontal displacement of the marker on the greater trochanter during one stride over the force-platform. To determine spatiotemporal gait characteristics a triaxial accelerometer was attached to the subjects' sacrum by means of double layered tape. 


\section{Study protocol}

All subjects wore shorts, walked barefoot and were allowed to familiarize themselves with the test settings. Measurements were performed at 2 gait velocities. At first the subjects were asked to complete 5 successful trials over the test track at their own preferred gait velocity. A trial was successful if the subject stepped on the platform with their right foot without aiming. As gait velocity affects plantar loading [24] and subjects with diabetes and polyneuropathy walk slower [19], all subjects were asked to complete 5 trails at a standardized, imposed gait velocity of 1.2 $\mathrm{m} / \mathrm{s}$, with a maximal deviation of 0.1 . This range resembles the preferred gait velocity for this age group [19]. Using an even smaller range would demand too many repetitions to reach 5 successful trials and would be too exhausting for the subjects.

\section{Analysis}

Plantar pressure data were masked in Novel Database Medical (13.3.42, Germany 2007) using the Novel 10 mask division (see fig 1). Trials were excluded if inconsistencies occurred in the automatic masking procedure, for example due to dragging of the hallux over the pressure-platform. For each trial an ASCII output was generated, that included the peak pressure (PP), the force-time integral and the contact area per foot region. Using Matlab (R2007b, USA 2007), the PTI was calculated as the quotient of the force-time integral and contact area for each mask [25]. The forefoot to rear foot ratio (F/R-ratio) was calculated by taking the quotient of the PTI of the forefoot (areas 3-10, see figure 1) and the heel (area1). The velocity of forward transfer of the COP (tCOP) was expressed as the percentage of the stance phase needed for the COP to reach the forefoot (area 310). Internal joint moments were obtained by means of inverse dynamics as applied in Savelberg et al. [11]. All joint moments were normalized for body mass. This included the maximal hip flexion and extension ( $\mathrm{H}$-flex, $\mathrm{H}$-ext) and maximal knee flexion and extension moment ( $\mathrm{K}$-flex, $\mathrm{K}$-ext), as well as the maximal, plantar flexion ankle joint moment (A-plant) and the plantar flexion ankle joint moments at $40 \%$ of the stance phase (A-40). This value of $40 \%$ was chosen based on earlier research that indicated that at this time point the ankle joint moment showed the largest differences between people with and without DPN [11]. Based on this research, the A-40 and Aplant were used in a quotient to characterize the ankle joint moment pattern in one value (A-40/Aplant).

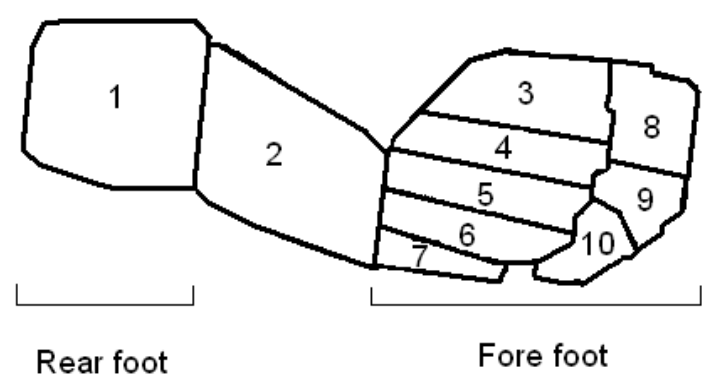

Figure 1. Mask division of plantar areas with indication of the areas used to calculate forefoot to rear foot ratio. Area $1=$ heel, area $2=$ mid foot, areas 3-7=metatarsal regions, area $8=$ hallux, areas 9 and $10=$ smaller toes. 
The duration of the stance phase was determined as the time the right foot was in contact with the force-platform. Stride length and stride duration were determined by assessing the duration of two successive peaks of the vertical acceleration signal and the average forward velocity (determined by the marker on the greater trochanter).

Averages for all variables were determined per subject based on a minimum of three correct trials.

\section{Statistical Analysis}

All data were analyzed using SPSS 15.0 for Windows. For both gait velocities separately, correlations between the A-40/A-plant, tCOP and F/R-ratio were determined using Pearson's Correlation Coefficient for all group together; these respective correlations were considered the main outcome of this study. Differences between groups were determined using a one way analysis of variance. Due to unequal variances in the different groups the Games-Howell test was used for post hoc analysis. Differences were considered significant if $p \leq 0.05$.

\section{Results}

Twenty-four subjects (21 DPN, 3 DC) were not able to reach the imposed gait velocity due to their health status. Hence, only their preferred gait velocity could be analyzed. Due to technical problems or incorrect loading of one of the platforms data were obtained in 117 subjects (72 DPN, $28 \mathrm{DC}, 17 \mathrm{HE}$ ) at the preferred gait velocity, and 91 subjects (56 DPN, $29 \mathrm{DC}, 16 \mathrm{HE}$ ) at the imposed gait velocity.

\section{Correlations between pressure characteristics and joint moments}

For the primary outcome measure all groups were analyzed together. Forwardly shifted foot pressures (F/R-ratio) correlated to higher displacement rates of COP (imposed gait velocity: $r=$ $-0.837, p<0.001$; preferred gait velocity: $r=-0.731, p<0.001)$. The displacement rate of the COP (tCOP) correlated with the relative plantar flexor moment (A-40/A-plant) of the first half of the stance phase (imposed gait velocity: $r=-0.749, p<0.001$; preferred gait velocity: $r=-0.693, p<$ 0.001 , Fig 2).

\section{Plantar pressure}

For the preferred gait velocity, DPN patients showed higher plantar loading in all areas under mid and forefoot in terms of PP and PTI than those in the HE group. At the imposed gait velocity the pressures (PTI and PP) under the mid foot and first and second metatarsal heads were significantly increased in DPN patients (table 2). In contrast to this increase of absolute loading in the forefoot, the data showed no differences between groups in displacement of COP or F/R-ratio.

\section{Joint moments}

For the preferred velocity, the post hoc analysis showed significantly higher values for A-40 and A40/A-plant for DPN compared to DC ( $p=0.003$ and $p=0.007$ respectively, table 2$)$. No group differences were found in joint moments for the imposed velocity. 


\section{Spatiotemporal gait characteristics}

Both DPN and DC patients preferred to walk slower than the healthy control subjects $(p=0.006$, table 2). In addition, during the preferred gait velocity, the DPN group tended to have a longer stride duration than the HE group $(p=0.061)$. Also in the DPN group the relative duration of stance phase was longer $(p=0.008)$ and subjects in this group took shorter strides than HE participants $(p=0.017)$. These differences were not present during the imposed gait velocity.
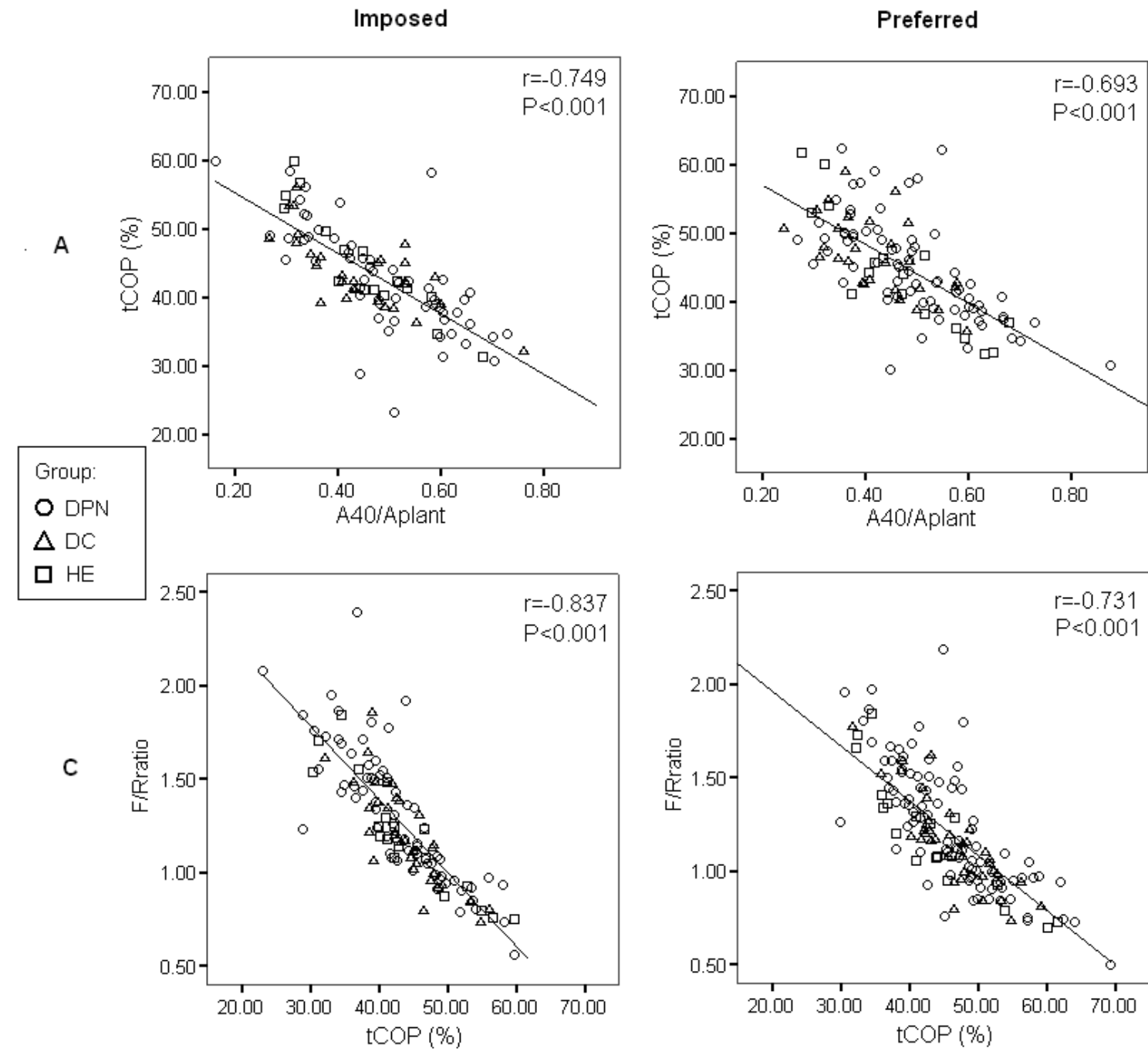

Figure 2. $A$ and B: Correlation of characterization of ankle joint moment ( $A-40 / A$-plant) and displacement rate of center of pressure (tCOP) for the imposed $(2 A)$ and preferred gait velocity $(2 B)$. A higher relative ankle joint moment at $40 \%$ of the stance phase is correlated with a faster forward transfer of the COP. C and D: Correlation of tCOP and the forefoot to rear foot PTI ratio (F/R ratio) for the imposed (2C) and preferred gait velocity (2D). A faster forward transfer of the COP results in a relatively earlier loading of the forefoot which is correlated with higher loading of the forefoot. Abbreviations: A-40: Ankle plantar flexion moment at 40\% of the stance phase; A-plant: Maximal ankle plantar flexion moment; tCOP: percentage of stance duration the center of pressure needs to reach the forefoot. F/R ratio: quotient of the pressure-time integral (PTI) under the forefoot and rear foot. 
Table 2. Means and standard errors of gait characteristics, internal joint moments, three main variables (A-40/A-plant, tCOP and F/R ratio) and plantar pressures (PP and PTI per foot region).

\begin{tabular}{|c|c|c|c|c|c|c|c|c|}
\hline & & Preferred & & & & Imposed & & \\
\hline & DPN & DC & HE & $\boldsymbol{P}$ & DPN & DC & HE & $\boldsymbol{P}$ \\
\hline Gait velocity (m/s) & $1.07(.02)^{b}$ & $1.09(.04)^{b}$ & $1.22(.04)$ & .006 & $1.23(.04)$ & $1.23(.06)$ & $1.23(.04)$ & .925 \\
\hline Stride duration (s) & $1.15(.01)$ & $1.13(.02)$ & $1.09(.02)$ & .061 & $1.04(.07)$ & $1.04(.08)$ & $1.07(.08)$ & .401 \\
\hline Stride length (m) & $1.26(01)^{a}$ & $1.22(.03)$ & $1.33(.03)$ & .045 & $1.28(.01)$ & $1.28(.02)$ & $1.31(.02)$ & .479 \\
\hline Stance phase & $.73(.01)^{b}$ & $.72(.01)$ & $.67(.02)$ & .017 & $.65(.05)$ & $.64(.05)$ & $.66(.05)$ & .664 \\
\hline $\mathrm{H}$-ext $(\mathrm{Nm} / \mathrm{kg})$ & $.66(.03)$ & $.62(.05)$ & $.79(.07)$ & .131 & $.74(.03)$ & $.65(.05)$ & $.74(.06)$ & .125 \\
\hline $\mathrm{H}$-flex $(\mathrm{Nm} / \mathrm{kg})$ & $.51(.03)$ & $.42(.05)$ & $.57(.07)$ & .163 & $.63(.03)$ & $.53(.05)$ & $.56(.06)$ & .770 \\
\hline K-ext (Nm/kg) & $.19(.03)$ & $.13(.02)$ & $.24(.04)$ & .210 & $.20(.03)$ & $.12(.02)$ & $.23(.04)$ & .678 \\
\hline $\mathrm{K}$-flex $(\mathrm{Nm} / \mathrm{kg})$ & $.43(.02)$ & $.48(.04)$ & $.52(.06)$ & .224 & $.53(.03)$ & $.57(.03)$ & $.54(.04)$ & .053 \\
\hline A-plant (Nm/kg) & $1.54(.02)$ & $1.55(.04)$ & $1.59(.04)$ & .529 & $1.57(.02)$ & $1.55(.04)$ & $1.58(.04)$ & .237 \\
\hline $\mathrm{A}-40(\mathrm{Nm} / \mathrm{kg})$ & $.76(.02)^{a}$ & $.65(.02)$ & $.74(.05)$ & .019 & $.75(.03)$ & $.67(.02)$ & $.71(.05)$ & .179 \\
\hline A-40/A-plant & $.49(.01)^{a}$ & $.42(.02)$ & $.47(.03)$ & .021 & $.48(.02)$ & $.45(.02)$ & $.45(.03)$ & .292 \\
\hline tCOP $(\%)$ & $46.0(.80)$ & $45.5(1.36)$ & $43.5(1.98)$ & .480 & $42.7(.89)$ & $44.3(.93)$ & $43.8(1.88)$ & .519 \\
\hline $\mathrm{F} / \mathrm{R}$ ratio & $1.24(.03)$ & $1.17(.04)$ & $1.20(.07)$ & .490 & $1.31(.04)$ & $1.20(.05)$ & $1.21(.07)$ & .222 \\
\hline PP Heel $\left(\mathrm{N} / \mathrm{cm}^{2}\right)$ & $38.1(1.1)$ & $36.0(1.6)$ & $36.0(2.6)$ & .517 & $42.9(1.4)$ & $40.5(1.9)$ & $36.9(2.3)$ & .126 \\
\hline PP Midfoot $\left(\mathrm{N} / \mathrm{cm}^{2}\right)$ & $15.4^{\mathrm{b}}(.6)$ & $15.6^{\mathrm{b}}(.8)$ & $12.0(.7)$ & .028 & $15.9(.7)^{b}$ & $15.6(.8)^{b}$ & $12.0(.5)$ & .023 \\
\hline PP MT1 $\left(\mathrm{N} / \mathrm{cm}^{2}\right)$ & $43.2^{b}(2.5)$ & $36.2(3.8)$ & $28.2(2.2)$ & .021 & $44.5(2.8)$ & $32.1(2.7)$ & $28.1(2.5)$ & .001 \\
\hline PP MT2 $\left(\mathrm{N} / \mathrm{cm}^{2}\right)$ & $57.1(2.6)$ & $47.1(3.8)$ & $45.9(4.4)$ & .039 & $60.7(3.0)^{\frac{a h}{b}}$ & $51.6(4.2)$ & $46.1(5.0)$ & .036 \\
\hline PP MT3 $\left(\mathrm{N} / \mathrm{cm}^{2}\right)$ & $46.9^{b}(1.8)$ & $40.9(2.5)$ & $36.4(1.5)$ & .011 & $49.0(2.3)^{\mathrm{b}}$ & $44.8(3.1)^{b}$ & $35.7(1.7)$ & .014 \\
\hline PP MT4 $\left(\mathrm{N} / \mathrm{cm}^{2}\right)$ & $31.3^{b}(1.2)$ & $27.5(1.2)$ & $24.2(1.0)$ & .007 & $31.5(1.7)^{b}$ & $28.5(1.2)$ & $25.3(1.7)$ & .101 \\
\hline PP MT5 $\left(\mathrm{N} / \mathrm{cm}^{2}\right)$ & $31.2^{\mathrm{ab}}(2.2)$ & $22.8(2.1)$ & $20.0(2.5)$ & .009 & $29.1(2.3)$ & $22.6(2.0)$ & $23.9(4.1)$ & .160 \\
\hline PP Hallux $\left(\mathrm{N} / \mathrm{cm}^{2}\right)$ & $44.4^{b}(2.6)$ & $45.8(4.5)$ & $33.6(2.6)$ & .178 & $45.0(3.0)^{b}$ & $49.6(5.0)^{b}$ & $34.1(3.4)$ & .099 \\
\hline PP Dig $2\left(\mathrm{~N} / \mathrm{cm}^{2}\right)$ & $20.6(1.4)$ & $19.3(1.7)$ & $20.9(2.1)$ & .824 & $21.4(1.5)$ & $20.6(1.8)$ & $20.7(2.3)$ & .932 \\
\hline PP Dig 3-5 (N/ $\left.\mathrm{cm}^{2}\right)$ & $14.1(.9)$ & $13.9(1.2)$ & $15.4(1.7)$ & .799 & $13.8(.8)$ & $15.94(1.6)$ & $15.2(1.8)$ & .405 \\
\hline PTI Heel $\left(\mathrm{Ns} / \mathrm{cm}^{2}\right)$ & $4.17(.13)^{b}$ & $4.02(.15)^{b}$ & $3.23(.19)$ & .004 & $3.47(.10)$ & $3.45(.11)$ & $3.12(.15)$ & .233 \\
\hline PTI Midfoot $\left(\mathrm{Ns} / \mathrm{cm}^{2}\right)$ & $1.69(.09)^{b}$ & $1.86(.14)^{b}$ & $.96(.09)$ & .001 & $1.35^{\mathrm{b}}(.08)$ & $1.47(.10)^{b}$ & $1.00(.08)$ & .032 \\
\hline PTI MT1 (Ns/cm²) & $5.18(.20)^{b}$ & $4.77(.24)^{b}$ & $3.45(.25)$ & $<.00$ & $4.76(.22)$ & $4.02(.19)$ & $3.30(.26)$ & .001 \\
\hline PTI MT2 (Ns/cm²) & $7.18(.19)^{b}$ & $6.55(.21)^{b}$ & $5.52(.31)$ & $<.00$ & $6.49(.20)^{\mathrm{ab}}$ & $5.99(.22)$ & $5.38(.28)$ & .015 \\
\hline PTI MT3 (Ns/cm²) & $6.59(.17)^{b}$ & $6.38(.24)^{b}$ & $5.15(.24)$ & $.00 \overrightarrow{1}$ & $5.83(.16)^{b}$ & $5.75(.23)$ & $5.05(.22)$ & .071 \\
\hline PTI MT4 (Ns/cm²) & $5.28(.17)^{b}$ & $5.30(.25)^{b}$ & $4.07(.25)$ & .007 & $4.49(.14)$ & $4.65(.22)$ & $4.12(.30)$ & .334 \\
\hline PTI MT5 (Ns/cm²) & $4.11(.20)^{b}$ & $3.78(.22)$ & $2.89(.30)$ & .023 & $3.29(.16)$ & $3.25(.19)$ & $3.07(.37)$ & .819 \\
\hline PTI Hallux (Ns/cm²) & $2.89(.13)$ & $2.90(.18)$ & $2.59(.17)$ & .577 & $2.59(.13)$ & $2.68(.18)$ & $2.52(.25)$ & .859 \\
\hline PTI Dig $2\left(\mathrm{Ns} / \mathrm{cm}^{2}\right)$ & $1.80(.10)$ & $1.66(.11)$ & $1.78(.17)$ & .675 & $1.57(.09)$ & $1.52(.12)$ & $1.80(.22)$ & .426 \\
\hline PTI Dig 3-5 (Ns/cm²) & $1.16(.07)$ & $1.21(.11)$ & $1.32(.17)$ & .626 & $.97(.07)$ & $1.06(.10)$ & $1.26(.17)$ & .178 \\
\hline
\end{tabular}

Differences based on post hoc testing: ${ }^{a}$ differs significantly from $D C,{ }^{b}$ differs significantly from HE. All joint moments are corrected for body mass. Abbreviations: H-ext: Maximum hip extension moment; H-flex: maximum hip flexion moment; $K$-ext: Maximum knee extension moment; A-plant: maximum plantar flexion moment; A-40: plantar flexion moment at 40\% of the stance phase; tCOP: the percentage of the stance phase needed for the center of pressure to reach the forefoot; PP: peak pressure; PTI: Pressure-time Integral; MT: metatarsal heads; Dig: digitorum; F/R ratio: Forefoot to rear foot PTI ratio.

\section{Discussion}

The aim of this study was to test the hypothesis that adaptations in force generation around the ankle joint result, via a cascade of relations, in a forwardly displaced plantar pressure pattern during gait. We observed that, irrespective of gait velocity, subjects who had a high A-40/A-plant had a faster forward transfer of the COP and showed simultaneously increased forefoot loading, as shown in figure 2 . This supports the hypothesis that the inability to generate enough force to brake the forward velocity during the first half of the stance phase contributes to a shift of pressure towards the forefoot. To the best of our knowledge, current study is the first to explicitly associate the force generating capacity by extrinsic muscles to plantar pressure patterns. This finding suggests that muscle weakness, a limited rate of force development, or delayed muscle activation 
contributes to adverse forefoot loading. It is possible that diminished functioning of the tibial anterior muscle causes a diminished control of the roll-off of the foot during loading response, resulting in a faster forward progression of the COP.

Also the plantar flexors are involved in braking the forward progression of COP, as these muscles eccentrically brake the forward progression of tibia during mid-stance. However, we observed in a previous study [11] that people with DPN have no apparent problem controlling the plantar flexion moment during the second half of stance phase, during which the role of the plantar flexors is more prominent. Future research needs to further clarify the contribution of the lower limb muscles to plantar pressures in DPN. Nevertheless, high plantar pressures are currently regarded as a local problem, and treated with shoe and offloading technology, but based on the results of the current study also other treatment options, such as training muscle strength or coordination, can be explored.

Based on previous literature $[20,21]$, it was expected that patients with DPN would have reduced muscle strength and thus would more frequently have a higher plantar flexion moment in early stance phase, limited braking capacity, a faster forward displacement of COP and higher loading of the forefoot [11]. In the current study the DPN patients did not show this outcome for the imposed gait velocity. The preferred gait velocity showed a higher A-40/A-plant for DPN patients compared to DC, but no increase in displacement of COP or forefoot loading. We did observe the cascade that connects ankle joints moments and loading of the forefoot in both diabetic groups, with and without DPN, and healthy subjects. This indicates that interventions aimed at increasing the control of the roll-off of the foot (e.g. training muscle strength or coordination) may contribute to the prevention of adverse plantar pressures patterns, even for people that are not at a direct risk for ulceration.

In the current study we observed group differences for spatiotemporal gait characteristics, plantar pressures and gait dynamics, which could have affected previously described cascade of relations.

The spatiotemporal gait characteristics of the current population showed that under the preferred gait velocity, people with DPN increased their stance phase duration and shorten their stride lengths. This finding is in concordance with previous observations. At preferred gait velocity Sawacha et al. [26] found increased stance phases and shorter stride lengths for people with diabetes, irrespective of the presence of polyneuropathy. Savelberg et al. [19] tested these same groups but did not observe any differences in spatiotemporal variables between groups; in addition they reported an influence of gait velocity on stride duration and stride length. This could mean that the longer stance phase duration and shorter stride lengths that were observed in the present study resulted from the lower gait velocity of participants with diabetes. The study of Sawacha et al. [26] showed indeed that people with diabetes walk slower than healthy subjects, again irrespective of the presence of polyneuropathy. The current study showed that when people with DPN where asked to walk at the same gait velocity as people without diabetes, the differences in gait characteristics disappeared. It is therefore assumed that the reported shorter stride length and longer stance phase duration found for people with diabetes and polyneuropathy are due to a lower, preferred gait velocity.

Significantly higher absolute loading, in terms of both PP and PTI, was found in the plantar forefoot of DPN patients. However, a forward shift of pressures could not be held responsible for 
this, as F/R-ratio and tCOP did not differ between groups. It is possible that increased absolute loading is due to structural and functional alterations as described by several authors [5-10]. Body mass is another factor that could lead to higher loading of the foot and therefore higher plantar pressures $[27,28]$. As the DPN group was significantly heavier than the other two groups, this will have led to differences in plantar pressures as well.

Regarding gait dynamics, our data showed an increased plantar flexion moment at $40 \%$ of the stance phase for people with DPN walking at their preferred gait velocity. Other joint moment variables, including the maximal plantar flexion, did not differ significantly over the groups. These findings conflict with the outcome of two earlier small studies. At preferred gait velocity, Mueller et al. [14] and Kwon et al. [15] found a decrease in plantar flexion joint moments for people with DPN with respect to $\mathrm{HE}$. Apart from the higher statistical power of the present study relative to these earlier studies (117 versus 10 and 9 respectively) we were not able to explain these differences in outcome.

It should be noted that the current study has some limitations. The subject groups were based on a convenience sample and were not matched or balanced, which might have influenced group differences. This possible bias was at least partly circumvented by the application of quotients for our main outcome variables on plantar pressure and joint moments. Even more, the correlation analysis was performed with all groups together, eliminating any possible bias in group characteristics.

In addition, for our inverse dynamic calculation no 3D kinematics were available. However a previous study indicated that the application of 2D kinematics is appropriate when analyzing human gait in the sagittal plain [29].

\section{Conclusion}

This study showed that a forward shift of plantar pressure is associated with a faster forward transfer of the COP, which is associated to a high, internal plantar flexion moment at 40 percent of the stance phase. It shows that force generation around the ankle joint plays an important role in the development of higher plantar pressures of the forefoot. Therefore it is to be expected that an intervention aimed at increasing the force generating abilities of the lower extremity can contribute to offloading of the forefoot. 


\section{References}

1. Boulton AJ, Hardisty CA, Betts RP, Franks CI, Worth RC, Ward JD, Duckworth T: Dynamic foot pressure and other studies as diagnostic and management aids in diabetic neuropathy. Diabetes Care 1983, 6(1):26-33.

2. Allet L, Armand S, Golay A, Monnin D, de Bie RA, de Bruin ED: Gait characteristics of diabetic patients: a systematic review. Diabetes Metab Res Rev 2008, 24(3):173-191.

3. Caselli A, Pham H, Giurini JM, Armstrong DG, Veves A: The forefoot-to-rearfoot plantar pressure ratio is increased in severe diabetic neuropathy and can predict foot ulceration. Diabetes Care 2002, 25(6): 1066-1071.

4. Mueller MJ, Zou D, Bohnert KL, Tuttle LJ, Sinacore DR: Plantar stresses on the neuropathic foot during barefoot walking. Phys Ther 2008, 88(11):1375-1384.

5. Boyko EJ, Ahroni JH, Stensel V, Forsberg RC, Davignon DR, Smith DG: A prospective study of risk factors for diabetic foot ulcer. The Seattle Diabetic Foot Study. Diabetes Care 1999, 22(7):1036-1042.

6. Mueller MJ, Hastings M, Commean PK, Smith KE, Pilgram TK, Robertson D, Johnson J: Forefoot structural predictors of plantar pressures during walking in people with diabetes and peripheral neuropathy. J Biomech 2003, 36(7):1009-1017.

7. Ahroni JH, Boyko EJ, Forsberg RC: Clinical correlates of plantar pressure among diabetic veterans. Diabetes Care 1999, 22(6):965-972.

8. Morag E, Cavanagh PR: Structural and functional predictors of regional peak pressures under the foot during walking. J Biomech 1999, 32(4):359-370.

9. Morag E, Pammer S, Boulton A, Young M, Deffner K, Cavanagh P: Structural and functional aspects of the diabetic foot. Clin Biomech (Bristol, Avon) 1997, 12(3):S9-S10.

10. Bus SA: Foot structure and footwear prescription in diabetes mellitus. Diabetes Metab Res Rev 2008, 24 Suppl 1:S90-95.

11. Savelberg HH, Schaper NC, Willems PJ, de Lange TL, Meijer K: Redistribution of joint moments is associated with changed plantar pressure in diabetic polyneuropathy. BMC Musculoskelet Disord 2009, $10: 16$.

12. Andersen $\mathrm{H}$, Poulsen $\mathrm{PL}$, Mogensen $\mathrm{CE}$, Jakobsen J: Isokinetic muscle strength in long-term IDDM patients in relation to diabetic complications. Diabetes 1996, 45(4):440-445.

13. Sacco IC, Amadio AC: A study of biomechanical parameters in gait analysis and sensitive cronaxie of diabetic neuropathic patients. Clin Biomech (Bristol, Avon) 2000, 15(3):196-202.

14. Mueller MJ, Minor SD, Sahrmann SA, Schaaf JA, Strube MJ: Differences in the gait characteristics of patients with diabetes and peripheral neuropathy compared with age-matched controls. Phys Ther 1994, 74(4):299-308; discussion 309-213.

15. Kwon OY, Minor SD, Maluf KS, Mueller MJ: Comparison of muscle activity during walking in subjects with and without diabetic neuropathy. Gait Posture 2003, 18(1):105-113.

16. van Deursen RW, Sanchez MM, Ulbrecht JS, Cavanagh PR: The role of muscle spindles in ankle movement perception in human subjects with diabetic neuropathy. Exp Brain Res 1998, 120(1):1-8.

17. Richardson JK, Ching C, Hurvitz EA: The relationship between electromyographically documented peripheral neuropathy and falls. J Am Geriatr Soc 1992, 40(10):1008-1012.

18. Abboud RJ, Rowley DI, Newton RW: Lower limb muscle dysfunction may contribute to foot ulceration in diabetic patients. Clin Biomech (Bristol, Avon) 2000, 15(1):37-45.

19. Savelberg HH, Ilgin D, Angin S, Willems PJ, Schaper NC, Meijer K: Prolonged activity of knee extensors and dorsal flexors is associated with adaptations in gait in diabetes and diabetic polyneuropathy. Clin Biomech (Bristol, Avon) 2010, 25(5):468-475.

20. IJzerman TH, Schaper NC, Melai T, Blijham P, Meijer K, Willems PJ, Savelberg HH: Motor nerve decline does not underlie muscle weakness in type 2 diabetic neuropathy. Muscle Nerve 2011, 44(2):241-245.

21. van Schie $\mathrm{CH}$, Vermigli $\mathrm{C}$, Carrington $\mathrm{AL}$, Boulton A: Muscle weakness and foot deformities in diabetes: relationship to neuropathy and foot ulceration in caucasian diabetic men. Diabetes Care 2004, 27(7):1668-1673.

22. Andreassen CS, Jakobsen J, Andersen $\mathrm{H}$ : Muscle weakness: a progressive late complication in diabetic distal symmetric polyneuropathy. Diabetes 2006, 55(3):806-812.

23. Valk GD, de Sonnaville JJ, van Houtum WH, Heine RJ, van Eijk JT, Bouter LM, Bertelsmann FW: The assessment of diabetic polyneuropathy in daily clinical practice: reproducibility and validity of Semmes Weinstein monofilaments examination and clinical neurological examination. Muscle Nerve 1997, 20(1):116-118.

24. Segal A, Rohr E, Orendurff M, Shofer J, O'Brien M, Sangeorzan B: The effect of walking speed on peak plantar pressure. Foot Ankle Int 2004, 25(12):926-933.

25. Melai T, Ijzerman TH, Schaper NC, de Lange TL, Willems PJ, Meijer K, Lieverse AG, Savelberg HH: Calculation of plantar pressure-time integral, an alternative approach. Gait Posture 2011, 34(3):379383.

26. Sawacha Z, Gabriella G, Cristoferi G, Guiotto A, Avogaro A, Cobelli C: Diabetic gait and posture abnormalities: a biomechanical investigation through three dimensional gait analysis. Clin Biomech (Bristol, Avon) 2009, 24(9):722-728.

27. Hills AP, Hennig EM, McDonald M, Bar-Or O: Plantar pressure differences between obese and nonobese adults: a biomechanical analysis. Int J Obes Relat Metab Disord 2001, 25(11):1674-1679.

28. Vela SA, Lavery LA, Armstrong DG, Anaim AA: The effect of increased weight on peak pressures: implications for obesity and diabetic foot pathology. J Foot Ankle Surg 1998, 37(5):416-420; discussion 448-419. 
29. Alkjaer T, Simonsen EB, Dyhre-Poulsen P: Comparison of inverse dynamics calculated by two- and three-dimensional models during walking. Gait Posture 2001, 13(2):73-77. 


\section{Chapter 5}

Lower leg muscle strengthening does not redistribute plantar load in diabetic polyneuropathy. A randomized controlled trial

Tom Melai, Nicolaas C. Schaper, T. Herman IJzerman, Ton L.H. de Lange, Paul J.B. Willems, Valéria Lima Passos, Aloysius G Lieverse, Kenneth Meijer, Hans H.C.M. Savelberg.

Journal of Foot and Ankle Research, 2013. Provisionally accepted for publication 


\section{Abstract}

Higher plantar pressures play an important role in the development of plantar foot ulceration in diabetic polyneuropathy and earlier studies suggested that the higher pressures under the forefoot may be related to a decrease in lower leg muscle strength. Therefore, in this randomized controlled trial we evaluated whether lower-extremity strength training can reduce plantar pressures in diabetic polyneuropathy, to reduce the risk of plantar ulceration.

This study was embedded in an unblinded randomized controlled clinical trial. Participants had diabetes and polyneuropathy and were randomly assigned to the intervention group ( $N=48)$ receiving strength training during 24 weeks, or the control group $(N=46)$ receiving no intervention. Plantar pressures were measured in both groups at $t=0, t=12, t=24$ and $t=52$ weeks. A random intercept model was applied to evaluate the effects of the intervention on peak pressures and pressure-time integrals, displacement of center of pressure and the forefoot to rearfoot pressure-time integral ratio.

Plantar pressure patterns were not affected by the strength training. In both the intervention and control groups the peak pressure and the pressure-time integral under the forefoot increased by $55.7 \mathrm{kPa}$ (CI: 14.7, 96.8) and $2.0 \mathrm{kPa} . \mathrm{s}(0.9,3.2)$ over 52 weeks, respectively. Both groups experienced high number of drop-outs, mainly due to deterioration of health status and lower-extremity disabilities.

Plantar pressures under the forefoot increase progressively over time in patients with diabetic polyneuropathy, but were in this study not affected by strength training. Future intervention studies should take this increase of plantar pressure into account and alternative interventions should be developed to reduce the progressive lower extremity problems in these patients. 


\section{Introduction}

Diabetic polyneuropathy (DPN) is an important risk factor for elevated plantar pressures and consequently the development of plantar foot ulceration [1, 2]. A foot ulcer is a feared complication of diabetes resulting in a high burden of disease for both the patient and the health care system. Diabetes is the leading cause of a lower extremity amputation in the world and more than $80 \%$ of these amputations are proceeded by a foot ulcer [3]. Although major improvements have been made in ulcer treatment, the results of interventions that aim to prevent these ulcers are still disappointing [4] and new preventive strategies are needed. In patients with DPN, especially the region of the forefoot is at higher risk due to deformities and altered gait patterns; higher loading of these areas has been associated with the development of plantar ulceration $[2,5]$. More specifically, Caselli et al.[2] found in DPN a higher forefoot to rear foot ratio (F/R-ratio) and concluded that this ratio could help to predict the occurrence of ulceration. Currently, most preventive strategies aim to reduce the abnormal loading of the foot in patients at risk by prescribing offloading footwear and aids like shoe inlays [6]. However, these interventions do not correct the underlying pathology and mixed results have been obtained [7].

Several authors suggested that muscle weakness, limited joint mobility, delayed muscle activation and impaired sensory-mechanical feedback contribute to higher plantar pressures in the forefoot [8-10]. In a previous study [11] we found that DPN patients had an impaired ability to brake the forward momentum of the body just after heel strike, leading to a faster roll-off of the foot and consequently a higher loading of the forefoot. We observed close correlations between the plantar flexion moment at the first part of the stance phase, the rate of forward progression of the center of pressure and loading of the forefoot [chapter 4]. These results suggest that the decreased muscle strength due to DPN $[12,13]$ can contribute to adverse plantar pressures. Therefore, we hypothesized that an intervention aimed at lower limb muscle strength could enable subjects to better counteract the forward momentum of the body during gait, thereby diminishing excessive forefoot loading. The aim of the current study is to evaluate the effects of lower extremity strength training on plantar pressures in DPN.

\section{Methods}

\section{Design overview}

The current study was embedded in a randomized clinical trial that was carried out in two institutes (trial registration: NCT00759265). The primary outcome measure of the trial was muscle function and the main secondary endpoint was mobility. Hence, sample size was determined based on the expected distribution and effects of exercise on muscle function, not on plantar pressures. Based on scarce literature $[14,15]$ the sample size of the trial was determined at 50 subjects with DPN for both the intervention and control group. The sample size of current study was based on this sample size determination and therefore can be considered a convenience sample. Due to practical reasons it was not possible for research staff and subjects to be blinded for treatment allocation. There is, to the best of our knowledge, little information on the effect of muscle strength training on plantar pressures in patients with DPN. Plantar pressure parameters were therefore included as 
secondary endpoints in the trial and will be reported in the current study. Outcome measures on muscle strength and mobility will be reported elsewhere.

\section{Setting and Participants}

Subjects were recruited from five diabetes outpatient clinics (one university and four regional hospitals) at the southern part of the Netherlands between December 2006 and November 2010. To be included, subjects had to be above 50 years of age and had to be able to walk 6 minutes without walking aids. Subjects were excluded if diagnosed with severe cardiac disease (NYHA $\geq 3)$, renal dysfunction (creatinin> $180 \mu \mathrm{mol} / \mathrm{l}$ ), intermittent claudication, neurological disorders other than DPN, rheumatoid arthritis, amputations, prior or current foot ulceration or were thought to be at risk of falling as assessed by the research staff. Subjects were also excluded if the presence of severe osteoarthritis or foot deformities interfered with the gait pattern.

The diagnosis of DPN was made in 94 subjects, scoring at least 4 points out of the maximal 33 during a standardized Clinical Neurological Examination (CNE), which included reflexes, light touch, vibration, position sense, pinprick and lower extremity muscle strength testing [16]. All participants signed an informed consent; the protocol was approved by the medical ethical committee of the hospitals involved.

All participants were tested in one of the two participating institutes. At both institutes a similar set-up was present with a 7 meter wooden walkway with an embedded pressure-platform (at the first institute: EMED-x, $100 \mathrm{~Hz}, 4$ sensors/ $\mathrm{cm}^{2}$, range 0-1270 kPa, Novel Inc., Munich, Germany. At the other institute: EMED-at, $50 \mathrm{~Hz}, 2$ sensors/ cm², range 0-1200 kPa, Novel Inc., Munich, Germany). Each subject was measured at the same institute and with the same platform throughout the study. Gait velocity was measured using custom made infrared detection gates placed 2 meters apart over the platform.

\section{Randomization and Intervention}

After baseline measurement, using the envelope method stratified over 5 groups ( 2 at the first institute and 3 the other), the participants were randomized by research staff, to either the intervention group receiving physical therapeutic training or to the control group receiving no training (figure1). Both groups were free to perform any additional physical activity outside the trial.

The subjects assigned to the intervention were divided over 5 sub-groups to receive weekly plenary training for 24 weeks. The intervention was developed by a multidisciplinary team, aiming to improve muscle strength of the lower limb. Every session was guided by a physical therapist under supervision of a member of the research team. During the first 12 weeks (part 1) the focus was on the lower leg, training of the dorsal and plantar flexors, and lower leg coordination. During the next 12 weeks (part 2) the entire lower extremity was trained.

Each training session consisted of four sections. The first section provided a warm up by simple exercises performed during gait. The second section included lower extremity strength training, gradually increasing from low to moderate intensity at 40 to $60 \%$ of one repeated maximum, and small, mostly seated, coordinative tasks. The materials used to build up resistance during these exercises were elastic bands, weight vests and ankle weights. The third section 
included functional gait tasks in terms of a challenging gait track with obstacles resembling activities of daily life. Each training lasted one and a half hour and was concluded with a selection of various interactive tailor fit games. In addition to these plenary sessions, subjects were provided with an exercise manual and were asked to carry on the exercises of the second section, two times a week at home, without supervision or monitoring. More detailed information on the training program has been made available in a supporting document (please see the appendix).

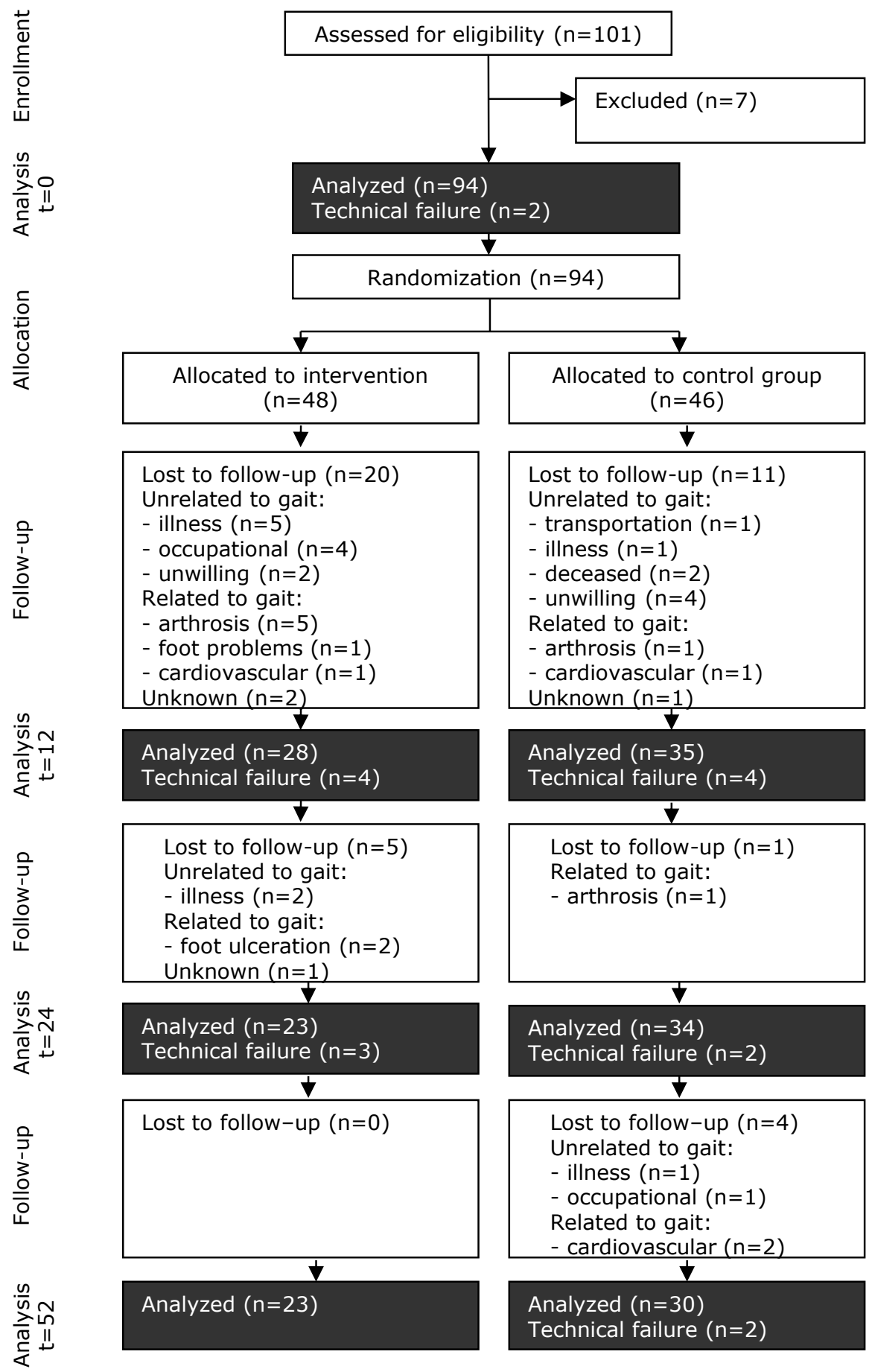

Figure 1. Flow diagram showing the design of the study and the number of subjects analyzed at different times of measurement (at $t=0,12,24$ and 52 weeks), including attrition due to technical failure. The lost to followup includes the reason for drop-out, which was classified in related or unrelated to gait. 


\section{Measurement protocol}

During measurements, all subjects wore shorts, walked barefoot and were allowed to familiarize themselves with the test settings of the plantar pressure measurements. Testing was performed at 2 gait velocities. At first the subjects were asked to walk over the test track at their own preferred gait velocity, which was allowed to vary each trial. Subsequently they had to complete the test at a standardized imposed gait velocity within a range of 1.1 to $1.3 \mathrm{~m} / \mathrm{s}$, as this resembles the preferred gait velocity for this age group [11]. At both velocities they had to complete preferably 5 , but at least 3 successful trials. A trial was successful if the subjects did not alter their gait pattern to target the platform, which was assessed by the research staff. For practical reasons and comparability over time only data was collected of the right foot.

Measurements were performed at baseline $(t=0)$, after the first part of the intervention, at 12 weeks ( $t=12)$, after the second part of the intervention, at 24 weeks $(t=24)$ and at follow-up, after 52 weeks $(t=52)$. If a subject did not wish to participate anymore in the study, they were asked to report the reason why. To monitor whether there was a possible relation with the intervention, the reasons given were classified as either gait-related or not gait-related (figure 1).

\section{Data analysis}

The effect of the training program on plantar pressures was considered the primary outcome of current study. Plantar pressure data were masked in Novel Database Medical (13.3.42, Germany 2007) using the Novel 10 mask division (area $1=$ heel, area $2=$ mid foot, areas $3-7=$ metatarsal region, area $8=$ hallux, area $9=$ second toe and area $10=$ smaller toes) [17]. If there were inconsistencies in the automatic masking procedure, due to for example dragging of the hallux over the pressure-platform, trials were excluded. For each trial the peak pressure, the force-time integral (FTI) and the contact area per foot region were calculated. Using Matlab (R2007b, USA 2007), the PTI was calculated as the quotient of the force-time integral and the contact area for each mask [17]. The PTI of the forefoot was calculated as a quotient of the summation of FTI for area 3 to 10 and the total contact area of these regions. Peak pressure of the forefoot was set as the highest peak pressure in area 3 to 10 . The forefoot to rear foot ratio (F/R-ratio) was calculated by taking the quotient of the PTI of the forefoot and the heel. The displacement rate of COP was operationalized as the percentage of the stance phase needed for the COP to reach the forefoot (area 3-10); this variable was abbreviated as tCOP. A minimum of 3 correct measurements were averaged for each subject per measurement $(t=0,12,24$ or 52$)$.

Biochemical analysis was performed to monitor overnight fasting blood glucose, glycated hemoglobin (HbA1C) and creatinin, at $\mathrm{t}=0,24$ and 52 .

\section{Statistical Analysis}

All data were analyzed using SPSS 15.0 for Windows and expressed as means with $95 \%$ confidence intervals (CI). The effect of the training program on the primary outcome was determined on an intention to treat analysis. As subjects participated in a training program with two components and were subsequently followed for 6 months, multiple measurements had to be performed over time. Given the hierarchical structure of the data, with repeated measurements nested within subjects, a random intercept model was fitted to evaluate average changes in plantar pressure variables over 
time for the intervention and control group, adjusting simultaneously for covariates. This model has the advantage of using all available observations for model estimation, inclusively those of subjects entered in the model until drop-out, and not only the complete cases like the classical repeated measures ANOVA.

Ideally both preferred and imposed gait velocity should be evaluated simultaneously. But, as not all subjects were able to complete the imposed gait velocity, this analysis was performed separately for the two gait velocities.

The time point at follow-up ( $t=52)$ was used as a reference for comparisons of involving temporal changes. Group, gender, body mass and age were entered as fixed factors. Subjects made up the random factor. The interaction between group and time was considered in order to test whether temporal changes of the outcomes over time depended on the group, with a significance level of $5 \%$. Estimates were determined by a restricted maximum likelihood method.

For interaction terms, whose $p$-values were $<0.1$, additional Likelihood Ratio (LR) tests were conducted (at $5 \%$ significance level). If non-significant, they were left out of the final model. All other fixed factors remained in the model, irrespective of significance.

It should be noted that in these multivariable models, the fixed effect parameters are unbiased, if attrition was non-informative, i.e. missingness conditioned on each covariate occurred at random. However, the pattern of missingness within groups, for instance, indicates that this condition may not hold. Therefore, results should be interpreted with caution (as will be discussed further on).

\section{Results}

\section{Subject characteristics}

Subjects were overall obese with a good to moderate glycemic control (table1). The majority was male and had moderate DPN (CNE score). Subjects in the control group were on average 3 years younger. All other characteristics did not differ significantly between groups (table1). At 52 weeks follow-up, both groups had the same body mass as at baseline, but increased their preferred gait velocity. At baseline measurement 21 subjects (intervention group: 12; control group: 9) were not able to reach the imposed gait velocity due to their health status. During the consecutive measurements the numbers of subjects that did not reach this faster gait velocity were 1,2 and 4 respectively for the intervention group and 6, 5 and 6 respectively for the control group.

Both groups experienced a high number of drop-outs during the first 12 weeks (figure1). In most cases attrition was caused by deterioration of health status prohibiting the subjects to further participate in the measurements or intervention. The reasons for drop-out as reported by participants are specified in figure1. Drop-out was in particular high for the intervention group in the first 12 weeks (Intervention: 41.7\%; Control: 22.2\%, $p=0.082$ ). The reason for stopping with the intervention was in 8 of the 20 patients who dropped-out, gait-related. In the control group 10 subjects dropped-out, 2 because of gait-related reasons. Throughout the full study, 11 of the 25 subjects stopped with the intervention because of gait-related problems, in the control group this number was 5 out of the 15 . 
Table 1. Subject characteristics at baseline

\begin{tabular}{lrr}
\hline & Intervention & Control \\
\hline Age (years) & $68.4(7.3)$ & $* 65.2(7.2)$ \\
Sex $($ male/female) & $40 / 8$ & $36 / 10$ \\
Length $(\mathrm{m})$ & $1.74(0.08)$ & $1.74(0.08)$ \\
Body Mass $(\mathrm{kg})$ & $92.2(20.1)$ & $94.2(19.1)$ \\
BMI $\left(\mathrm{kg} \cdot \mathrm{m}^{2}\right)$ & $30.4(6.2)$ & $30.9(4.9)$ \\
CNE score & $12.9(4.9)$ & $12.8(5.3)$ \\
HbA1c $(\%)$ & $7.3(1.1)$ & $7.3(1.1)$ \\
Glucose $(\mathrm{mmol} / \mathrm{L})$ & $8.8(2.6)$ & $8.2(2.0)$ \\
Creatinine $(\mu \mathrm{mol} / \mathrm{L})$ & $100.9(35.1)$ & $94.8(23.5)$ \\
\hline
\end{tabular}

Means and standard deviation of the measured subject characteristics at baseline for both intervention and control group. Age determined at onset of study. Abbreviations: CNE= Clinical Neurological Examination. * Significant difference between intervention and control group $(p<0.05)$.

\section{Effect of training on plantar loading}

No significant effect of the intervention on the plantar pressures characteristics (peak pressures, PTI, tCOP and F/R-ratio) was observed (LR $p$-values $>0.1$ ). As the interaction effect (time*group) was for none of the variables significant it was left out of subsequent models. Therefore, the random intercept model was constructed of age, gender (male versus female), body mass, group (intervention versus control) and time of measurement ( $t=0,12$ and 24 , versus 52 weeks).
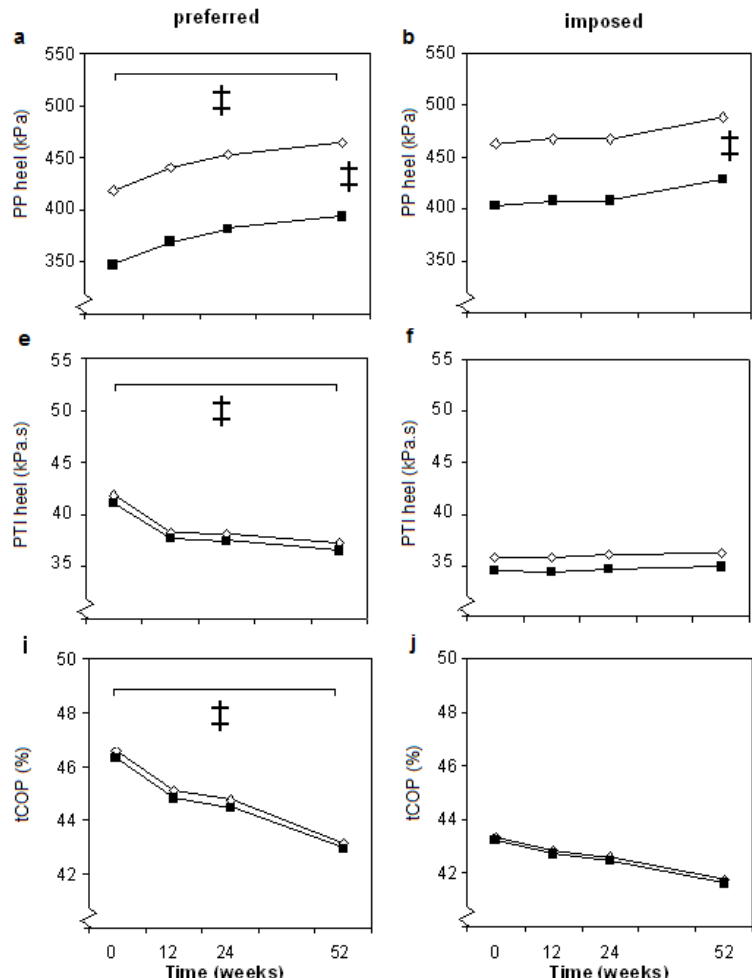
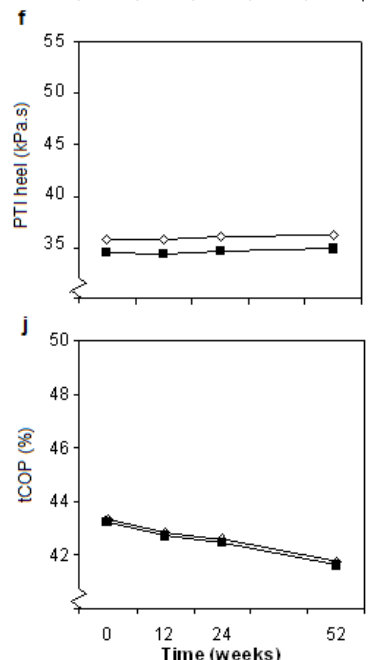
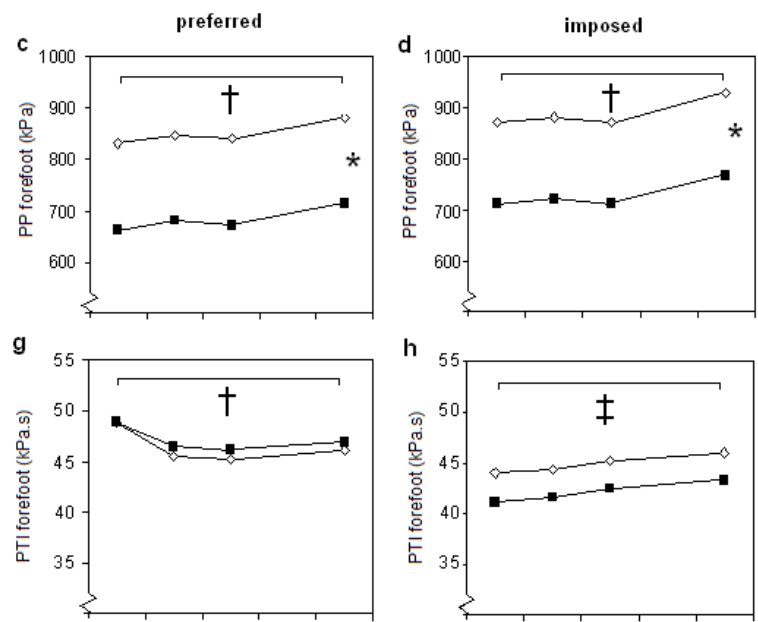

k

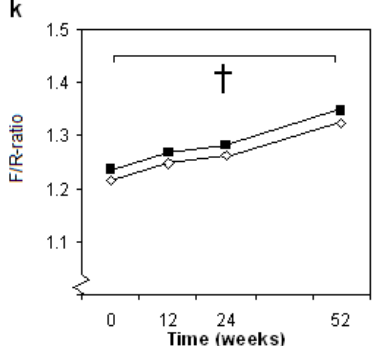

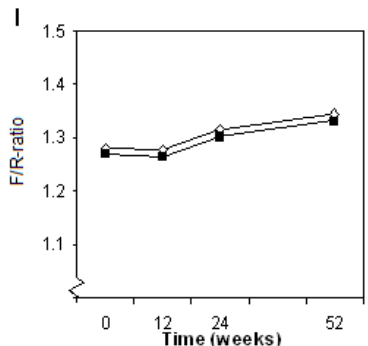

Figure 2(a-I). Estimated mean values over time for intervention (in black) and control groups (in white) of all outcome parameters according to the final random intercept models (table2). Graphs have broken y-axes to improve readability. PP is peak pressure; PTI is pressure-time integral; tCOP is center of pressure expressed as percentage of the stance phase for it to enter the forefoot; F/R-ratio is the forefoot to rear foot PTI ratio. Significant time effects over 52 weeks and group differences are marked with: $*=p \leq 0.05, t=p \leq 0.01, \neq=p \leq$ 0.001 . 


\section{Effect of time on plantar loading}

Time effects were present for almost all plantar pressure parameters, regardless of the group. At the preferred gait velocity, the peak pressure under the heel increased over 52 weeks with 45.8 $\mathrm{kPa}(95 \% \mathrm{CI}: 25.3,66.2 ; p \leq 0.001 ;$ table and figure 2a). At the preferred and imposed gait velocity, the peak pressure under the forefoot increased in both groups with 52.8 (CI: 18.0, 87.5) and 55.7 (CI: $14.7,96.8) \mathrm{kPa}$, respectively ( $p \leq 0.01$; table and figure $2 \mathrm{c}-\mathrm{d}$ ). The PTI of the heel and forefoot decreased in both groups with 4.5 (CI: 2.6, 6.7) and 2.0 (CI: 0.4, 3.5) kPa.s, respectively ( $p \leq 0.01$; table and figure $2 \mathrm{e}$ and $\mathrm{g}$ ), at the preferred gait velocity. In contrast, at the imposed gait velocity the PTI increased under the forefoot in both groups with $2.0 \mathrm{kPa} . \mathrm{S}$ (CI: 0.9 , $3.2 ; p \leq 0.01$; table and figure $2 \mathrm{~h}$ ). Moreover, tCOP decreased in both groups during the preferred gait velocity with $3.4 \%(\mathrm{CI}: 1.92,4.90 ; p \leq 0.01)$ as shown in the table and figure $2 \mathrm{i}$ and $j$, indicating a faster forward transfer of COP. Also F/R-ratio increased over time for the preferred gait velocity with 0.11 (CI: $0.18,0.38$; table and figure $2 \mathrm{k} ; p \leq 0.01$ ).

Table 2(a-I). The determinants and their effects on plantar pressures in diabetic patients with polyneuropathy depicted as $\hat{\beta}$ (CI 95)

\begin{tabular}{|c|c|c|c|c|}
\hline & Preferred & Imposed & Preferred & \multirow{2}{*}{$\begin{array}{l}\text { Imposed } \\
\text { ot (kPa.s) }\end{array}$} \\
\hline & a. PP heel (kPa) & b. PP heel (kPa) & c. PP forefoot (kPa.s) & \\
\hline Intercept & $358.6(120.0,597.1)^{\dagger}$ & $149.2(-110.0,408.3)$ & $955.1(396.6,1513.5) \neq$ & $706.4(84.5,1328.3)^{*}$ \\
\hline Age & $-2.3(-5.1, .6)$ & $0.1(-3.2,3.4)$ & $-4.6(-11.4,2.1)$ & $-1.4(-9.2,6.4)$ \\
\hline Body mass & $2.1(.8,3.4) \dagger$ & $3.0(1.6,4.3) \neq$ & $-1.1(-2.0,4.1)$ & $2.1(-1.2,5.3)$ \\
\hline Group (IG vs CG) & $72.0(33.7,110.3) \neq$ & $60.4(20.9,99.8) \dagger$ & $168(82.8,253.5)^{*}$ & $162.4(70.9,253.9)^{*}$ \\
\hline Time $t=0$ vs 52 & $-45.8(-66.2,-25.3) \neq$ & $-25.6(-45.9,-5.3)$ & $-52.8(-87.5,-18.0) \dagger$ & $-55.7(-96.8,-14.7) \dagger$ \\
\hline Time $t=12$ vs 52 & $-23.7(-45.1,-2.5)^{*}$ & $-20.5(-41.0,0.1)$ & $-36.0(-71.5,-.3)^{*}$ & $-46.4(-87.7,-5.2)^{*}$ \\
\hline Time $t=24$ vs 52 & $-11.7(-33.3,9.9)$ & $-20.5(-41.4,0.4)$ & $-42.4(-78.4,-6.4)^{*}$ & $-55.4(-97.4,-13.3)^{*}$ \\
\hline$\sigma_{i}^{2}$ & $296.7(237.7,370.3) \ddagger$ & $218.0(169.9,279.6) \neq$ & $821.1(655.7,1028.2) \ddagger$ & $871.6(676.3,1123.3) \neq$ \\
\hline \multirow[t]{2}{*}{$\sigma_{b_{0}}^{2}$} & $868.2(611.9,1232.0) \neq$ & $897.9(623.8,1292.4) \neq$ & $5858.9(4225.9$ & $5848.5(4084.7$ \\
\hline & e. PTI heel (kPa.s) & f. PTI heel (kPa.s) & g. PTI forefoot & h. PTI forefoot \\
\hline Intercept & $3.6(-21.8,29.0)$ & $31.9(14.3,49.3) \neq$ & $8.2(-8.0,24.4)$ & $25.3(10.0,40.6) \dagger$ \\
\hline Age & $.1(-.2, .4)$ & $-.3(-.6,-.1)^{\dagger}$ & $.2(-.0,0.4)$ & $-.8(-.3,1.2)$ \\
\hline Body mass & $.3(.1, .4) \neq$ & $.3(.2, .4) \neq$ & $.3(.2, .4) \neq$ & $.2(.2, .3) \neq$ \\
\hline Group (IG vs CG) & $.7(-3.3,4.8)$ & $1.3(-1.5,4.0)$ & $-.8(-3.5,1.8)$ & $2.8(.5,5.1)$ \\
\hline Time $t=0$ vs 52 & $4.6(2.6,6.7)^{\ddagger}$ & $-.3(-2.0,1.4)$ & $2.0(.4,3.5) \dagger$ & $-2.0(-3.2,-0.9) \ddagger$ \\
\hline Time $t=12$ vs 52 & $1.1(-1.0,3.3)$ & $-.4(-2.2,1.3)$ & $-.6(-2.2,1.1)$ & $-1.7(-2.9,-.5) \dagger$ \\
\hline Time $t=24$ vs 52 & $.9(-1.3,3.1)$ & $-.1(-1.9,1.6)$ & $-.8(-2.5, .8)$ & $-0.8(-1.9, .4)$ \\
\hline$\sigma_{i}^{2}$ & $3.0(2.4,3.7) \neq$ & $1.6(1.2,2.0) \neq$ & $1.7(1.4,2.2) \ddagger$ & $.7(.5, .9) \neq$ \\
\hline \multirow[t]{2}{*}{$\sigma_{b_{0}}^{i}$} & $10.3(7.2,15.6) \neq$ & $3.5(2.3,5.2) \neq$ & $3.7(2.5,5.5) \neq$ & $3.2(2.2,4.7) \neq$ \\
\hline & i. $\operatorname{tcop}(\%)$ & j. tCOP (\%) & k. F/R-ratio & I. F/R-ratio \\
\hline Intercept & $33.16(15.05,51.28) \neq$ & $51.58(35.05,68.11) \neq$ & $1.31(0.52,2.09) \dagger$ & $.93(.07,1.80)^{*}$ \\
\hline Age & $.03(-.19, .25)$ & $-.26(-.47,-.06) \dagger$ & $.00(-.01, .01)$ & $.01(-.00,0.02)$ \\
\hline Body mass & $.09(-.01,0.19)$ & $0.09(0.01,0.18)^{*}$ & $-.00(-.01, .00)$ & $-.00(-.01, .01)$ \\
\hline Group (IG vs CG) & $.26(-2.64,3.15)$ & $.12(-2.48,2.72)$ & $-.02(-.15, .11)$ & $.01(-.12, .15)$ \\
\hline Time $t=0$ vs 52 & $3.41(1.92,4.90) \neq$ & $1.60(-0.03,3.22)$ & $-.11(-.18,-.38) \dagger$ & $-.06(-.14, .021)$ \\
\hline Time $t=12$ vs 52 & $1.93(.39,3.48)^{*}$ & $1.07(-.60,2.74)$ & $-.08(-.15,-.02)^{*}$ & $-.07(-.15, .019)$ \\
\hline Time $t=24$ vs 52 & $1.59(.03,3.16)^{*}$ & $.83(-.87,2.53)$ & $-.06(-.14, .01)$ & $-.03(-.11, .06)$ \\
\hline$\sigma_{i}^{2}$ & $15.64(12.53,19.51)^{\ddagger}$ & $14.48(11.31,18.54) \neq$ & $0.04(0.03,0.05) \neq .000$ & $.04(.03, .05) \neq$ \\
\hline$\sigma_{b_{n}}^{2}$ & $51.49(36.53,72.58) \neq$ & $30.95(20.80,46.04) \neq$ & $0.09(0.06,0.13) \neq .000$ & $.09(.06, .13) \ddagger$ \\
\hline
\end{tabular}

Measurements were obtained at two gait velocities, preferred and imposed (see text). Estimated fixed (and random) effect parameters of the random intercept models are shown. $\beta$ (95\% CI) represent average changes in the outcome variables per unit increase for the continuous explanatory variables. For categorical variables, the estimates represent average changes with respect to the reference (control group, females, or follow-up measurement at $t=52$ weeks). Effects of time are negative if baseline measurements were lower than during follow-up. Age was determined at onset of the study. Abbreviations and symbols: $I G=$ intervention group; $C G=$ control group; $M=$ male; $F=$ Female; $P P=$ peak pressure; $*=p \leq 0.05, t=p \leq 0.01$. 


\section{Overall group differences}

At all times of measurement, there were no differences between the intervention and control group for PTI, tCOP and F/R-ratio, but the peak pressures under heel and forefoot were significantly higher for the intervention group for both the preferred and imposed gait velocity ( $p \leq 0.01$, table and figure $2 a-d)$.

\section{Effects of covariates}

For every extra year of age, PTI of the heel was lower ( $p \leq 0.01$, table $2 f)$ and the COP reached the forefoot earlier ( $p \leq 0.01$, table $2 \mathrm{j}$ ) at the imposed gait velocity. An increase in body mass of one $\mathrm{kg}$ was associated for both the preferred and imposed gait velocity, with increased loading of the heel in terms of peak pressure and PTI ( $p \leq 0.01$, table2 a-d). The same was observed for the PTI under the forefoot for both gait velocities ( $p \leq 0.01$, table $2 \mathrm{~g}$ and $\mathrm{h}$ ). Gender did not have a significant effect on one of the outcome parameters and was for the sake of clarity omitted from table 2.

\section{Discussion}

In the current study we tested the effect of a gait and strength training program on plantar pressure distribution in DPN. Our main finding is that the exercise program did not affect plantar pressure patterns. As we did not observe a difference between the intervention and control groups we could combine both arms in a prospective observational study, in which we observed an increase in forefoot loading over time in subjects with DPN, during the individually preferred and standardized imposed gait velocity.

As far as we know only one other study examined the effects of exercise training on plantar pressures; in postmenopausal women a reduction in plantar peak pressures at preferred gait velocity was observed after 12 months training but the underlying mechanism of this reduction was not provided [18]. It is unclear why our intervention did not affect plantar pressure patterns. The high drop-out rate might have influenced the outcome, but to be reported elsewhere, the intervention did increase maximal plantar flexion strength, although maximal dorsal flexion strength was not affected (IJzerman et al. Unpublished data). The lack of effect on dorsal flexor strength and plantar pressures could be explained by either insufficient intensity of the training or impaired trainability due to the underlying neuropathy of DPN patients. Alternatively the increased forefoot loading in DPN may not originate from muscle weakness per se, but rather from abnormalities in other factors associated with joint motion, motor control, feedback from muscle spindles, rate of force development and sensory nerve function of the skin [10, 19-21].

Our study resulted in some important findings that should be taken into account in future research exploring the effects of other or similar interventions on plantar pressures in patients with DPN. First of all, it is possible that we did not observe an effect of our intervention, due to a large between-subjects variability. In our research we included patients with different types of diabetic polyneuropathy, several mild comorbidities and several levels of motivation. To see which intervention is most suitable for the patients being helped, future research needs to focus on strictly defined specific patient groups before looking at general benefits of an intervention in a RCT. 
Secondly, we observed an increase in forefoot plantar pressure over time in both the intervention and control group. These changes in pressure distribution differed between the preferred and imposed gait velocity conditions. During the preferred gait velocity, peak pressure under the heel and forefoot increased and the PTI under heel and forefoot decreased over time. The COP reached the forefoot earlier and F/R-ratio was higher after one year. These changes over time are probably related to the increased gait velocity in our subjects when subjects could determine their own gait velocity. An increased gait velocity would lead to shorter plantar loading and therefore a lower PTI. Although all subjects were familiarized with the experimental conditions, it is possible that this increased gait velocity was caused by habituation to the experimental settings.

A different pattern of the plantar pressures over time was seen when gait velocity was kept constant. During the standardized imposed gait velocity, both the peak pressure and PTI under the forefoot increased over time in subjects with DPN. Our data clearly indicate that gait velocity should be taken into account in future studies and when studying mechanisms of elevated plantar pressures or the effect of interventions, gait velocity should be standardized in at least one set of measurements.

Earlier studies also reported an increase of plantar pressures over time [1,2], but only a few provided quantitative information. It has been reported that during a mean follow-up of 34 months mean plantar pressure increased with $65 \mathrm{kPa}$ in people with diabetes type 2 unspecified for neuropathy status [22]. In subjects with type 1 or type 2 diabetes and DPN plantar pressure increased with $255 \mathrm{kPa}$ over a mean follow-up of 30 months [23]. Both studies did not report whether they controlled or monitored gait velocity. In this study we observed in subjects with type 2 diabetes and DPN an increase after 12 months under the forefoot of $2.0 \mathrm{kPa}$.s for the PTI and 55.4 kPafor the peak pressure (table $2 \mathrm{~h}$ and $\mathrm{d}$ ), during the imposed gait velocity. High plantar pressures are an important risk factor for foot ulceration and in one prospective study patients who developed an ulcer during the 2-year observation period had a mean baseline peak pressure of 955 $\pm 264 \mathrm{kPa}$, while non-ulcerated subjects had a peak pressure of $851 \pm 273 \mathrm{kPa}(p<0.001)[24]$. The difference of $100 \mathrm{kPa}$ between the ulcerated and non-ulcerated subjects was less than 2 times the increase of pressure we observed in one year. The risk for ulceration in patients with DPN seems therefore to increase rapidly over time.

Other information that could be useful for future research can be obtained from the limitations of the current study. A major limitation was the high drop-out rate, especially during the first 12 weeks of the training program. The application of a random intercept model circumvented, at least partly, the problem of missingness irrespective of the differential attrition rate between the groups. Via this modeling approach, the estimated mean values of the outcome parameters, contingent on the model covariates, can be considered unbiased if the pattern of missingness within each experimental group were at random. In the intervention group more subjects dropped out because of gait-related problems, which might have introduced bias in the final parameter estimates. Progression of polyneuropathy often leads to progression of morbidity [25] and an increase in plantar loading [2]. Therefore, it is conceivable that subjects that refrained from the study had higher plantar pressures than the subjects that completed the study.

The higher drop-out rate could not be directly attributed to overuse injuries, but we cannot rule out the possibility that the training contributed to deterioration of the health status of participants that dropped out. Gait-related problems were a major reason for drop-out in one third 
of the patients who withdrew from the study and in addition the staff had the impression that lack of motivation also played an important role in several patients. The motivation to exercise is probably suboptimal in many patients with diabetes, as persons that are less motivated to exercise throughout their lives have a higher chance to develop diabetes and its complications [26, 27]. A high drop-out rate was also a major problem in other long term exercise programs in patients with diabetes, e.g. a drop-out rate of $60 \%$ was observed during brisk walking program [18, 28, 29]. To prevent drop-out in future interventions, more emphasis should be placed on the prevention of gait-related problems, on improving patient selection criteria with respect to health status and motivation and on keeping them motivated. Finally, in power calculations the high drop-out rate should be taken into account.

Another limitation of the current study is that sample size was determined based on the expected distribution and effects of exercise on muscle function, not on plantar pressures. Also since no correction for multiple testing was applied, significant findings should be interpreted with caution. The study was set-up with a single blinded design, outcome assessors being blinded for the allocation of participants. However, as subjects were not able to conceal their allocation, blinding of assessors could not be realized. But, plantar loading was measured with an objective procedure and during the data analysis only subject codes were used that could not be related to treatment allocation. Finally, it could be considered a limitation that we did not use identical pressure-platforms at the two study sites, which in theory could have affected the outcome. Nevertheless, we expect the difference in platforms to be negligible, as the platforms are of the same manufacturer, provide comparable data and in particular because our primary outcome was the change in plantar pressure.

In conclusion, a lower extremity strength training program was not successful in reducing plantar loading of the forefoot for people with diabetic polyneuropathy. Forefoot loading increased in both the intervention and control group over time resulting in a progressive rise in the risk of foot ulceration. The results described in this article provide valuable data for the design of future mechanistic and intervention studies. 


\section{References}

1. Allet L, Armand S, Golay A, Monnin D, de Bie RA, de Bruin ED: Gait characteristics of diabetic patients: a systematic review. Diabetes Metab Res Rev 2008, 24(3):173-191.

2. Caselli A, Pham H, Giurini JM, Armstrong DG, Veves A: The forefoot-to-rearfoot plantar pressure ratio is increased in severe diabetic neuropathy and can predict foot ulceration. Diabetes Care 2002, 25(6):1066-1071.

3. Pecoraro RE, Reiber GE, Burgess EM: Pathways to diabetic limb amputation. Basis for prevention. Diabetes Care 1990, 13(5):513-521.

4. Valk GD, Kriegsman DM, Assendelft WJ: Patient education for preventing diabetic foot ulceration. A systematic review. Endocrinology and metabolism clinics of North America 2002, 31(3):633-658.

5. Mueller MJ, Zou D, Bohnert KL, Tuttle LJ, Sinacore DR: Plantar stresses on the neuropathic foot during barefoot walking. Phys Ther 2008, 88(11):1375-1384.

6. Bus SA, Valk GD, van Deursen RW, Armstrong DG, Caravaggi C, Hlavacek P, Bakker K, Cavanagh PR: Specific guidelines on footwear and offloading. Diabetes Metab Res Rev 2008, 24 Suppl 1:S192-193.

7. Bus SA, Valk GD, van Deursen RW, Armstrong DG, Caravaggi C, Hlavacek P, Bakker K, Cavanagh PR: The effectiveness of footwear and offloading interventions to prevent and heal foot ulcers and reduce plantar pressure in diabetes: a systematic review. Diabetes Metab Res Rev 2008, 24 Suppl 1:S162180.

8. Abboud RJ, Rowley DI, Newton RW: Lower limb muscle dysfunction may contribute to foot ulceration in diabetic patients. Clin Biomech (Bristol, Avon) 2000, 15(1):37-45.

9. Kwon OY, Minor SD, Maluf KS, Mueller MJ: Comparison of muscle activity during walking in subjects with and without diabetic neuropathy. Gait Posture 2003, 18(1):105-113.

10. Savelberg HH, Ilgin D, Angin S, Willems PJ, Schaper NC, Meijer K: Prolonged activity of knee extensors and dorsal flexors is associated with adaptations in gait in diabetes and diabetic polyneuropathy. Clin Biomech (Bristol, Avon) 2010, 25(5):468-475.

11. Savelberg HH, Schaper NC, Willems PJ, de Lange TL, Meijer K: Redistribution of joint moments is associated with changed plantar pressure in diabetic polyneuropathy. BMC Musculoskelet Disord 2009, 10:16.

12. IJzerman TH, Schaper, N. C., Melai T., Meijer K., Willems P.J.B., Savelberg H.H.C.M. : Lower extremity muscle strength is reduced in people with type 2 diabetes, with and without polyneuropathy, and is associated with impaired mobility and reduced quality of life. . Diabetes Research and Clinical Practice 2011, doi:10.1016/j.diabres.2011.10.026.

13. van Schie $\mathrm{CH}$, Vermigli C, Carrington AL, Boulton A: Muscle weakness and foot deformities in diabetes: relationship to neuropathy and foot ulceration in caucasian diabetic men. Diabetes Care 2004, 27(7):1668-1673.

14. Kouidi E, Albani M, Natsis K, Megalopoulos A, Gigis P, Guiba-Tziampiri O, Tourkantonis A, Deligiannis A: The effects of exercise training on muscle atrophy in haemodialysis patients. Nephrol Dial Transplant 1998, 13(3):685-699.

15. Chetlin RD, Gutmann L, Tarnopolsky M, Ullrich IH, Yeater RA: Resistance training effectiveness in patients with Charcot-Marie-Tooth disease: recommendations for exercise prescription. Arch Phys Med Rehabil 2004, 85(8):1217-1223.

16. Valk GD, de Sonnaville JJ, van Houtum WH, Heine RJ, van Eijk JT, Bouter LM, Bertelsmann FW: The assessment of diabetic polyneuropathy in daily clinical practice: reproducibility and validity of Semmes Weinstein monofilaments examination and clinical neurological examination. Muscle Nerve 1997, 20(1):116-118.

17. Melai T, Ijzerman TH, Schaper NC, de Lange TL, Willems PJ, Meijer K, Lieverse AG, Savelberg HH: Calculation of plantar pressure-time integral, an alternative approach. Gait Posture 2011, 34(3):379383.

18. Monteiro MA, Gabriel RE, Neves ECM, Sousa MF, Abrantes JM, Moreira MH: Exercise effects in plantar pressure of postmenopausal women. Menopause 2010,17(5):1017-1025.

19. Delbridge L, Perry P, Marr S, Arnold N, Yue DK, Turtle JR, Reeve TS: Limited joint mobility in the diabetic foot: relationship to neuropathic ulceration. Diabet Med 1988, 5(4):333-337.

20. van Deursen RW, Sanchez MM, Ulbrecht JS, Cavanagh PR: The role of muscle spindles in ankle movement perception in human subjects with diabetic neuropathy. Exp Brain Res 1998, 120(1):1-8.

21. Courtemanche R, Teasdale N, Boucher P, Fleury M, Lajoie Y, Bard C: Gait problems in diabetic neuropathic patients. Arch Phys Med Rehabil 1996, 77(9):849-855.

22. Grimm A, Kastenbauer T, Sauseng S, Sokol G, Irsigler K: Progression and distribution of plantar pressure in Type 2 diabetic patients. Diabetes Nutr Metab 2004, 17(2):108-113.

23. Veves A, Murray HJ, Young MJ, Boulton AJ: The risk of foot ulceration in diabetic patients with high foot pressure: a prospective study. Diabetologia 1992, 35(7):660-663.

24. Lavery LA, Armstrong DG, Wunderlich RP, Tredwell J, Boulton AJ: Predictive value of foot pressure assessment as part of a population-based diabetes disease management program. Diabetes Care 2003, 26(4):1069-1073.

25. Miralles-Garcia JM, de Pablos-Velasco P, Cabrerizo L, Perez M, Lopez-Gomez V: Prevalence of distal diabetic polyneuropathy using quantitative sensory methods in a population with diabetes of more than 10 years' disease duration. Endocrinol Nutr 2010, 57(9):414-420.

26. Gill JM, Cooper AR: Physical activity and prevention of type 2 diabetes mellitus. Sports Med 2008, 38(10):807-824. 
27. Hu FB, Leitzmann MF, Stampfer MJ, Colditz GA, Willett WC, Rimm EB: Physical activity and television watching in relation to risk for type 2 diabetes mellitus in men. Archives of internal medicine 2001, 161(12):1542-1548.

28. Praet SF, van Rooij ES, Wijtvliet A, Boonman-de Winter LJ, Enneking T, Kuipers H, Stehouwer CD, van Loon LJ: Brisk walking compared with an individualised medical fitness programme for patients with type 2 diabetes: a randomised controlled trial. Diabetologia 2008, 51(5):736-746.

29. Thoolen B, de Ridder D, Bensing J, Gorter K, Rutten G: Who participates in diabetes self-management interventions?: Issues of recruitment and retainment. Diabetes Educ 2007, 33(3):465-474. 


\section{Chapter 6}

Strength training affects lower extremity gait kinematics, not kinetics, in people with diabetic polyneuropathy

Tom Melai, Nicolaas C. Schaper, T. Herman IJzerman, Paul J.B. Willems, Ton L.H. de Lange, Kenneth Meijer, Aloysius G Lieverse, Hans H.C.M. Savelberg.

Journal of Applied Biomechanics, 2013. Accepted for publication 


\section{Abstract}

Increased forefoot loading in diabetic polyneuropathy plays an important role in the development of plantar foot ulcers and can originate from alterations in muscle strength, joint moments and gait pattern. The current study evaluated whether strength training can improve lower extremity joint moments and spatiotemporal gait characteristics in patients with diabetic polyneuropathy.

An intervention group receiving strength training during 24 weeks and a control group receiving no intervention. Measurements were performed in both groups at $t=0, t=12, t=24$ and $\mathrm{t}=52$ weeks at an individually preferred and standardized imposed gait velocity.

The strength training did not affect the maximal amplitude of hip, knee and ankle joint moments, but did result in an increase in stance phase duration, stride time and stride length of approximately $5 \%$, during the imposed gait velocity. In addition, both groups increased their preferred gait velocity over one year.

Future longitudinal studies should further explore the possible effects of strength training on spatiotemporal gait characteristics. The current study provides valuable information on changes in gait velocities and the progressive lower extremity problems in patients with polyneuropathy. 


\section{Introduction}

People with diabetic polyneuropathy (DPN) experience higher loading of the forefoot, which is a risk factor for the development of ulcers [1]. Several authors suggested that the presence of muscle weakness or limitations in motor control could play a role in the development of this increased forefoot loading [2-4]. In a previous study [5] we found a higher ankle plantar flexion moment during the first half of the stance phase in DPN patients with higher loading of the forefoot. In these patients a faster forward displacement of the point of application of the ground reaction force (GRF) concurred with an increase of the internal ankle plantar flexion moment and a decrease of the internal knee extension moment [5]. In a subsequent study we observed strong positive associations between the internal ankle plantar flexion moment, the roll-off of the foot and forefoot loading [6]. The dorsal flexors play an important role in decelerating the plantar flexion movement just after heel strike and the plantar flexors limit forward displacement of the tibia by eccentric contraction in late mid stance, causing delayed forward displacement of center of pressure and thereby limiting the ankle plantar flexion moment. Therefore, we hypothesized that muscle weakness of these muscle groups and limitations in motor control result in patients with DPN in an impaired ability to compensate the ankle plantar flexion moment during gait, leading to higher forefoot loading. In addition to the changes in joint moments, also changes in spatiotemporal gait characteristics can influence plantar loading in DPN [7]. People with DPN have a lower gait velocity, take smaller steps and have a longer stride duration $[8,9]$, which can lead to lower peak pressures. These changes in spatiotemporal gait characteristics can be a result of muscle weakness or limitations in motor control and will, in part, counterbalance the effects of the altered joint moments, as this would lead to a lower loading of the forefoot.

We hypothesize that improving lower extremity muscle strength should enable a better control of the roll-off of the foot, a slower forward displacement of the point of application of the GRF and consequently a lower ankle plantar flexion moment during the first part of the stance phase and a higher knee extension moment. Therefore, the aim of the current study was to evaluate the effects of strength training on lower extremity joint moments in patients with DPN. As the training might lead to changes in spatiotemporal gait characteristics, which can influence joint moments, also the effects on gait velocity, stance phase duration, stride length and stride duration were evaluated. In addition, as gait velocity is an important confounder of joint moments and spatiotemporal gait characteristics, current study was performed at both a preferred and a standardized, imposed gait velocity.

\section{Methods}

This study was part of a randomized controlled multi-center trial (registration: NCT00759265) with two different study sites, both in the southern part of the Netherlands.

\section{Participants}

Participants were recruited from five diabetes outpatient clinics (one university and four regional hospitals) and all measurements were performed at two study sites. Participants had to be able to 
walk independently for six minutes and had to be between 50 and 80 years of age, to create a homogenous group of patients with type 2 diabetes. Participants were excluded if diagnosed with severe cardiac disease, renal dysfunction (creatinin $>180 \mu \mathrm{mol} / \mathrm{l}$ ), intermittent claudication, neurological disorders other than DPN, rheumatoid arthritis, severe osteoarthritis, foot deformities, amputations or foot ulceration. Participants needed to be able to participate in gait experiments without risk of falling, which was assessed by the research staff. The diagnosis of DPN was made in 94 participants, scoring at least 4 points out of the maximal 33 during a standardized Clinical Neurological Examination (CNE), which included reflexes, light touch, vibration, position sense, pinprick and lower extremity muscle strength testing [10].

At each study site, using sealed envelopes, the participants were randomized to the intervention group, receiving strength training or to the control group receiving no training (figure 1). Both groups were free to perform any additional physical activity or training outside the trial.

\section{Intervention}

Patients assigned to the intervention received a weekly plenary training, which was designed based on scarce literature and multidisciplinary consensus [11], aiming to improve motor control and muscle strength of the lower extremity in a 24 weeks program guided by a physical therapist. In the first 12 weeks (part one) the training focused on the muscles around the ankle. During the next 12 weeks (part two) also the muscles around the knee were trained.

Each plenary training session lasted one and a half hour and started with a warm up by simple exercises during gait, followed by lower extremity muscle strength training. Resistance was built up from low to moderate intensity at 40 to $60 \%$ of one repeated maximum, based on the number of repetitions until fatigue, using elastic bands, weight vests and ankle weights. In addition, small coordinative tasks (e.g. moving objects with their feet, narrow based walking) were performed in order to train motor control of the lower extremity. During part one of the training, especially dorsal and plantar flexion strength were trained in isolated and chained conditions. During part two, also training of knee extension and flexion strength were added. Next, participants received gait training on an obstacle course, set-up to resemble activities of daily life. The plenary training session was concluded with a selection of various interactive, tailor fit games. In addition to the supervised sessions once a week, participants were asked to carry on two times a week the muscle strength training exercises at home. More information on the training program is provided in a supplementary document (please see the appendix).

\section{Experimental set-up}

At both study sites a seven meter wooden walkway with an embedded force-platform was used; at one site an AMTI force-platform was used (AMTI LG6_2-1,Advanced Medical Technology Inc, Newton, MA, US) and at the other site a Kistler platform was available (9281A SN, Kistler instrumente AG, Winterhur, Switzerland). Throughout the study, each subject was measured at the same institute and with the same platform. At both sites the same digital high speed camera (1.3 megapixel Pixellink PL-A741, sampling at $100 \mathrm{~Hz}$ ) recorded the 2D position of the reflective markers in the sagittal plane. These markers were attached to the skin at the middle of the greater trochanter, lateral femoral epicondyle, calcaneus, lateral malleolus and fifth metatarsal of the right 
leg of each subject, as described earlier [5]. Average horizontal displacement of the marker on the greater trochanter was used to determine gait velocity. An indication of gait velocity during the trial was obtained by means of photocells placed over the platform. Spatiotemporal gait characteristics were determined using a tri-axial accelerometer (MT-9, Xsense, Enschede, the Netherlands) that was attached to the participants' sacrum by means of double layered tape.

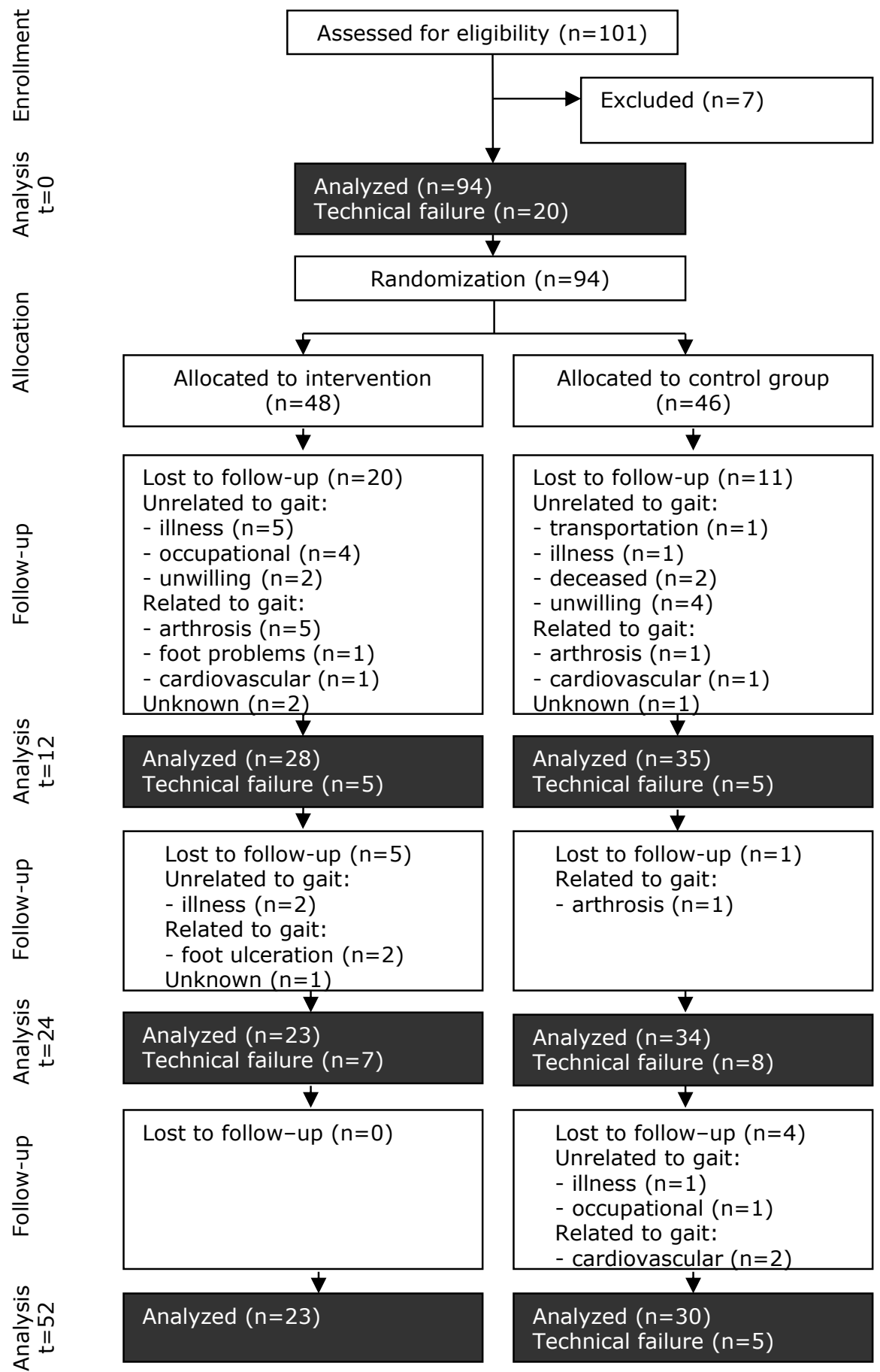

Figure 1. Flow chart illustrating the study design, the number of subjects analyzed at different times of measurement (at $t=0,12,24$ and 52 weeks) and attrition due to technical failure (e.g. incomplete marker sets, interference of the other foot). Loss of follow-up was specified with reason for drop-out, which was classified as related or unrelated to gait. 


\section{Measurement protocol}

The protocol was approved by the medical ethical committee of Maastricht University Medical Center+ and all participants signed an informed consent.

Participants were asked to walk with bare feet over de test track as naturally as possible. A trial was successful if the participants did not alter their gait pattern to target the platform with their right foot. After completing five successful trials at their preferred gait velocity, participants were asked to complete again five successful trials at a standardized, imposed gait velocity at 1.2 $\mathrm{m} / \mathrm{s}$, as this resembles the preferred gait velocity for this age group [5]. For practical reasons we allowed a range in gait velocity of $+/-0.1 \mathrm{~m} / \mathrm{s}$.

Participants were measured at baseline before randomization $(t=0)$, at 12 weeks $(t=12)$, after the first part of the intervention, at 24 weeks $(t=24)$ after the second part of the intervention, and after 52 weeks at follow up $(t=52)$. Due to practical reasons it was not possible to perform measurements by research staff blinded for treatment allocation. When a subject choose to cease their participation, they were asked to report the reason (if they wished so), the reasons for withdrawal were classified as either gait or not gait related.

As hyperglycemia can affect muscle performance [12], fasting blood glucose and glycated hemoglobin, HbA1c were measured at $\mathrm{t}=0, \mathrm{t}=24$ and $\mathrm{t}=52$.

\section{Analysis}

The primary outcome measures for this study were the effects of the strength training program on lower limb gait characteristics, including joint moments, stance phase duration, stride length and stride duration. The effects of time, age, body mass and sex on these parameters were secondary outcome measures.

An inverse dynamics approach was used to obtain internal joint moments [5]. For characterization of individual joint moments, maximal internal extension and flexion moments at hip (H-ext, $\mathrm{H}$-flex), knee (K-ext, $\mathrm{K}$-flex) and ankle joint (A-plant) were determined. Also the ankle plantar flexion moments at $40 \%$ of the stance phase $(A-40)$ were assessed. To characterize the pattern of the ankle joint moment, the quotient of A-40 over A-plant was calculated (A-40/A-plant). Previous research indicated that this quotient discriminates well between people with and without DPN [5]. As body mass is an important confounder of the forces applied during gait, all joint moments were normalized for body mass [13].

Stance phase duration was defined as the contact time of the right foot on the forceplatform. The time between two successive peaks of the vertical acceleration signal and gait velocity were used to calculate stride length and stride duration.

The average of each variable was determined using a minimum of three correct trials per subject per time of measurement.

\section{Statistical Analysis}

SPSS 15.0 for Windows was used for data analysis. Data are presented as mean with either standard deviation or $95 \%$ confidence interval (CI). A random intercept model was fitted to evaluate the effect of the intervention and changes in gait characteristics over time, adjusting simultaneously for covariates. Velocity was not entered into the model as not all participants were 
able to complete the imposed gait velocity. Therefore, analysis was performed separately for both the preferred and imposed gait velocity. Age, gender, body mass and group were entered as fixed factors for model estimation of the spatiotemporal gait parameters. If not significant, the factor body mass was left out of the model estimation for joint moments as these already included a correction for this factor. The random factor was made up by the participants. An interaction term between group and time was considered for all parameters in order to test whether temporal changes of the outcomes over time depended on the intervention. Additionally, the effect of study site on the interaction of group and time was explored to test whether this could have influenced the effect of the intervention. If this was not the case, study site was left out of the model. All fixed factors with exception of interaction of group and time, were always left in the model. Analyses were performed on an intention to treat basis and all available measurements were used for model estimation, including the measurements of the patients who dropped out. Differences were considered significant if $p \leq 0.05$. Estimates were determined by a restricted maximum likelihood method. As long as missing occurs at random, the advantage of the mixed model approach is that our analyses would not be affected by missing data and that the fixed effect parameters are unbiased. However, the pattern of missing data within the groups indicates that this condition may not hold. Significant differences should therefore be interpreted with caution. Finally, the outcome measures as reported in this article were secondary endpoints of a study of strength training on mobility in DPN.

Table 1. Subject characteristics at baseline measurement

\begin{tabular}{|c|c|c|c|}
\hline & IG & CG & $p$-value \\
\hline Age (years) & $68.2(7.3)$ & $65.5(7.4)$ & 0.962 \\
\hline Sex (male/female) & $40 / 8$ & $36 / 10$ & 0.217 \\
\hline Body height $(\mathrm{m})$ & $1.74(0.08)$ & $1.74(0.08)$ & 0.913 \\
\hline Body mass (kg) & $91.6(20.3)$ & $94.3(18.9)$ & 0.789 \\
\hline BMI $\left(\mathrm{kg} \cdot \mathrm{m}^{2}\right)$ & $30.2(6.3)$ & $30.9(4.9)$ & 0.190 \\
\hline CNE score & $12.9(4.9)$ & $13.0(5.5)$ & 0.186 \\
\hline HbA1c (\%) & $7.3(1.1)$ & $7.3(1.1)$ & 0.796 \\
\hline Glucose (mmol/L) & $8.8(2.6)$ & $8.2(2.0)$ & 0.426 \\
\hline Creatinine $(\mu \mathrm{mol} / \mathrm{L})$ & $100.6(34.7)$ & $95.9(24.5)$ & 0.076 \\
\hline
\end{tabular}

Means and standard deviation of the subject characteristics and biochemical analysis at baseline for both intervention and control group. Age determined at onset of study. Abbreviations: CNE= Clinical Neurological Examination; IG = intervention group; $C G=$ control group.

\section{Results}

There were no baseline differences between the intervention and control group (IG and CG respectively, table 1). Participants were elderly (IG 68.2 years, CG 65.5 years) with moderate DPN (CNE score; 12.9 and 13.0, respectively). Most of the participants were men (83 and 78\%), had moderate glycemic control (HbA1c; 7.3 and 7.3\%) and were overweight to obese (BMI; 30.2 and $30.9 \mathrm{~kg} \cdot \mathrm{m}^{-2}$ ). Overall, participants did not experience changes in body mass during the study. At baseline, 16 participants (IG 8, CG 8) were not able to reach the imposed gait velocity, for these participants only measurements of their preferred gait velocity were analyzed. The number of participants that were not able to reach the faster, imposed velocity during the three follow up measurements was respectively 5, 2 and 4 for the intervention group, and 8,5 and 2 for the 
control group. In 17 cases data could not be analyzed due to technical failure, such as incomplete marker sets or interference of the other foot (figure 1 ).

Drop-out was high in both groups, especially during the first 12 weeks (IG 41.7\%; CG $22.2 \%)$. The reasons for drop-out are reported in figure 1 . Gait related reasons were reported for 8 of the 20 participants that stopped with the intervention during the first 12 weeks. In the control group this was 2 out of 10 . Throughout the complete study 25 participants stopped the intervention of which 11 reported gait related problems. In the control group 5 out of 15 participants dropped out due to such problems. Probably due to the relative small number of participants for Chi-square analysis, no significant differences between the 2 groups were observed in drop-outs.

In comparison to the CG patients, the training program resulted in the IG patients in an increase in stride length of $5.1 \%(0.065 \mathrm{~m}, p \leq 0.04$, table and figure $2 \mathrm{~d})$, in stance phase duration of $5.5 \%(0.035 \mathrm{~s}, p \leq 0.01$, table and figure $2 \mathrm{f})$ and in stride time of $5.6 \%(0.058 \mathrm{~s}, p \leq 0.01$, table and figure $2 \mathrm{~h}$ ) determined during the standardized, imposed gait velocity. For the other variables,

Table 2(a-n). The determinants and their effects on spatiotemporal gait characteristics in diabetic patients with polyneuropathy depicted as $\hat{\beta}$ (CI 95).

\begin{tabular}{|c|c|c|c|c|}
\hline & $\begin{array}{r}\text { Preferred } \\
\text { a. Gait Velocity } \\
(\mathbf{m} / \mathbf{s})\end{array}$ & $\begin{array}{r}\text { Imposed } \\
\text { b. Gait Velocity } \\
(\mathbf{m} / \mathbf{s})\end{array}$ & $\begin{array}{r}\text { Preferred } \\
\text { c. Stride Length } \\
(\mathbf{m})\end{array}$ & $\begin{array}{r}\text { Imposed } \\
\text { d. Stride Length } \\
(\mathbf{m})\end{array}$ \\
\hline Intercept & $1.849(1.502,2.197) \neq$ & $1.322(1.185,1.458) \neq$ & $1.766(1.453,2.080)^{\ddagger}$ & $1.494(1.225,1.764) \neq$ \\
\hline Age (years) & $-.008(-.012,-.004) \neq$ & $-.001(-.003, .000)$ & $-.006(-.010,-.002) \dagger$ & $-.003(-.007, .000)$ \\
\hline Gender (F/ M) & $-.027(-.117, .062)$ & $-.008(-.041, .024)$ & $.001(-.080, .083)$ & $.008(-.058, .075)$ \\
\hline Body mass (kg) & $-.002(-.004, .000)$ & $-.000(-.001, .001)$ & $-.001(-.003, .000)$ & $.000(-.002, .001)$ \\
\hline Group (IG vs CG) & $.034(-.025, .093)$ & $.016(-.008, .038)$ & $.047(-.007, .100)$ & $.069(.009, .129)+$ \\
\hline Time $t=0$ vs 52 & $-.093(-.135,-.052) \neq$ & $.010(-.011, .032)$ & $-.063(-.097, .029) \neq$ & $.006(-.035, .047)$ \\
\hline Time $t=12$ vs 52 & $-.037(-.079,-.006)$ & $.008(-.014, .030)$ & $-.021(-.055, .014)$ & $-.026(-.066, .015)$ \\
\hline Time $t=24$ vs 52 & $-.016(-.060, .028)$ & $.007(-.016, .029)$ & $-.004(-.040, .032)$ & $.003(-.038, .043)$ \\
\hline Group*Time t=0 vs 52 & & & & $-.065(-.125,-.005)^{*}$ \\
\hline Group*Time $t=12$ vs 52 & & & & $.031(-.031, .093)$ \\
\hline Group*Time $t=24$ vs 52 & & & & $.004(-.059,066)$ \\
\hline$\sigma_{i}^{2}$ & $.010(.008, .013) \ddagger$ & $.002(.002, .003) \ddagger$ & $.007(.005, .009) \neq$ & $.004(.003, .006) \neq$ \\
\hline \multirow[t]{2}{*}{$\sigma_{b_{0}}^{2}$} & $.015(.010, .023) \neq$ & $.001(.001, .003) \dagger$ & $.013(.009, .020) \neq$ & $.008(.005,0.13) \neq$ \\
\hline & $\begin{array}{l}\text { e. Stand Phase } \\
\text { Duration (s) }\end{array}$ & $\begin{array}{l}\text { f. Stand Phase } \\
\text { Duration (s) }\end{array}$ & g. Stride Time (s) & h. Stride Time (s) \\
\hline Intercept & $.435(.266, .604) \neq$ & $.701(.580, .823) \neq$ & $.797(.571,1.02) \neq$ & $1.144(.963,1.325) \neq$ \\
\hline Age (years) & $.003(.001, .005) \dagger$ & $-.001(-.002, .001)$ & $.004(.001, .006) \dagger$ & $-.001(-.004, .001)$ \\
\hline Gender (F/ M) & $.023(-.020, .067)$ & $.012(-.018, .042)$ & $.044(-.015, .102)$ & $.016(-.028, .060)$ \\
\hline Body mass (kg) & $.001(-.000, .002)$ & $.000(-.001, .001)$ & $.000(-.001, .002)$ & $-.000(-.001, .001)$ \\
\hline Group (IG vs CG) & $-.010(-.039, .019)$ & $.021(-.005, .048)$ & $-.008(-.046, .031)$ & $.038(-.004, .080)$ \\
\hline Time $t=0$ vs 52 & $.027(.007, .047)$ & $-.011(-.029, .007)$ & $.043(.015, .070)^{\dagger}$ & $-.002(-.033, .028)$ \\
\hline Time $t=12$ vs 52 & $.011(-.010, .031)$ & $-.013(-.030, .005)$ & $.022(-.006, .050)$ & $-.020(-.050, .010)$ \\
\hline Time $t=24$ vs 52 & $.008(-.013, .030)$ & $-.005(-.023, .012)$ & $.016(-.013, .045)$ & $-.007(-.037, .023)$ \\
\hline Group*Time t=0 vs 52 & & $-.035(-.061, .008) \dagger$ & & $-.058(-.102,-.014)^{*}$ \\
\hline Group*Time $t=12$ vs 52 & & $-.004(-.031, .023)$ & & $-.006(-.040, .052)$ \\
\hline Group*Time $t=24$ vs 52 & & $.005(-.023, .032)$ & & $.017(-.029, .064)$ \\
\hline$\sigma_{i}^{2}$ & $.002(.002, .003) \neq$ & $.001(.001, .001)^{\ddagger}$ & $.005(.004, .006) \neq$ & $.002(.002, .003) \neq$ \\
\hline$\sigma_{b_{0}}^{2}$ & $.004(.002, .005) \neq$ & $.002(.001, .003) \neq$ & $.006(.004, .010) \neq$ & $.003(.002, .006) \neq$ \\
\hline
\end{tabular}

Note. Measurements were obtained at two gait velocities, preferred and imposed (see text). Estimated fixed (and random) effect parameters of the random intercept models are shown. $\beta(95 \%$ CI) represent average changes in the outcome variables per unit increase for the continuous explanatory variables. For categorical variables, the estimates represent average changes with respect to the reference (control group, females, or follow up measurement at $t=52$ weeks). Age was determined at onset of the study. Significant differences are marked with: * $p \leq 0.05 ;+p \leq 0.01 ; \neq p \leq 0.001$. Abbreviations: IG= intervention group; $C G=$ control group; $M=$ male; $F=$ Female. 
no differences between the CG and IG patients were observed after baseline, in particular, no differences were observed in joint moments. As training did not affect these variables, an interaction effect was not included in the model studying these parameters. In addition, no interaction of study site with group status was observed, it was therefore left out of the model as well.

Regardless of the effect of the training program, effects of time on the CG and IG groups combined were present for several parameters, suggesting either a learning effect and/or deterioration over time. After 52 weeks, both the intervention and control group walked $8.8 \%$ faster under the preferred gait velocity condition (0.093 $\mathrm{m}^{-1} \mathrm{~s}^{-1} p<0.001$ figure/ table 2a), taking $5.3 \%$ larger strides $(0.063 \mathrm{~m}, p<0.001$ figure and table $2 \mathrm{c})$ in $3.7 \%$ less time $\left(0.043 \mathrm{~m} . \mathrm{s}^{-1}, p \leq\right.$ 0.01 figure and table $2 \mathrm{~g}$ ). A-40 increased with $8.6 \%$ during the preferred gait velocity (0.062 N.m. $\mathrm{kg}^{-1}, p \leq 0.01$, figure and table 3i). In addition, the maximal hip joint extension moment increased with $15.2 \%$ ( $0.097 \mathrm{~N} . \mathrm{m}_{\mathrm{kg}}{ }^{-1}, p \leq 0.01$, figure and table $\left.3 a\right)$ and the maximal hip joint flexion moment increased with 21.6\% (0.112 N.m. $\mathrm{kg}^{-1}, p \leq 0.01$, figure and table $3 \mathrm{c}$ ) in our patients irrespective of their training status during the preferred gait velocity after 52 weeks. Also maximal knee extension moment increased with $33.8 \%$ during the preferred gait velocity $(0.149$ N.m. kg ${ }^{-1}, p<0.001$, figure and table $3 e$ ) and $19.4 \%$ during the imposed gait velocity (0.102 N.m. kg-1, $p<0.001$, figure and table 3f), while maximal knee flexion moment decreased with $47.5 \%$ during the preferred gait velocity $\left(0.089 \mathrm{~N} . \mathrm{m}_{\mathrm{kg}} \mathrm{k}^{-1}, p<0.001\right.$, figure and table $3 \mathrm{~g}$ ) and $55.2 \%$ during the imposed gait velocity (0.109 N.m. kg-1,$p<0.001$, figure and table $3 \mathrm{~h}$ ).

Age, body mass and sex were found to influence joint moments and spatial temporal gait characteristics in our multivariate model. Other covariates did not significantly affect the studied parameters.
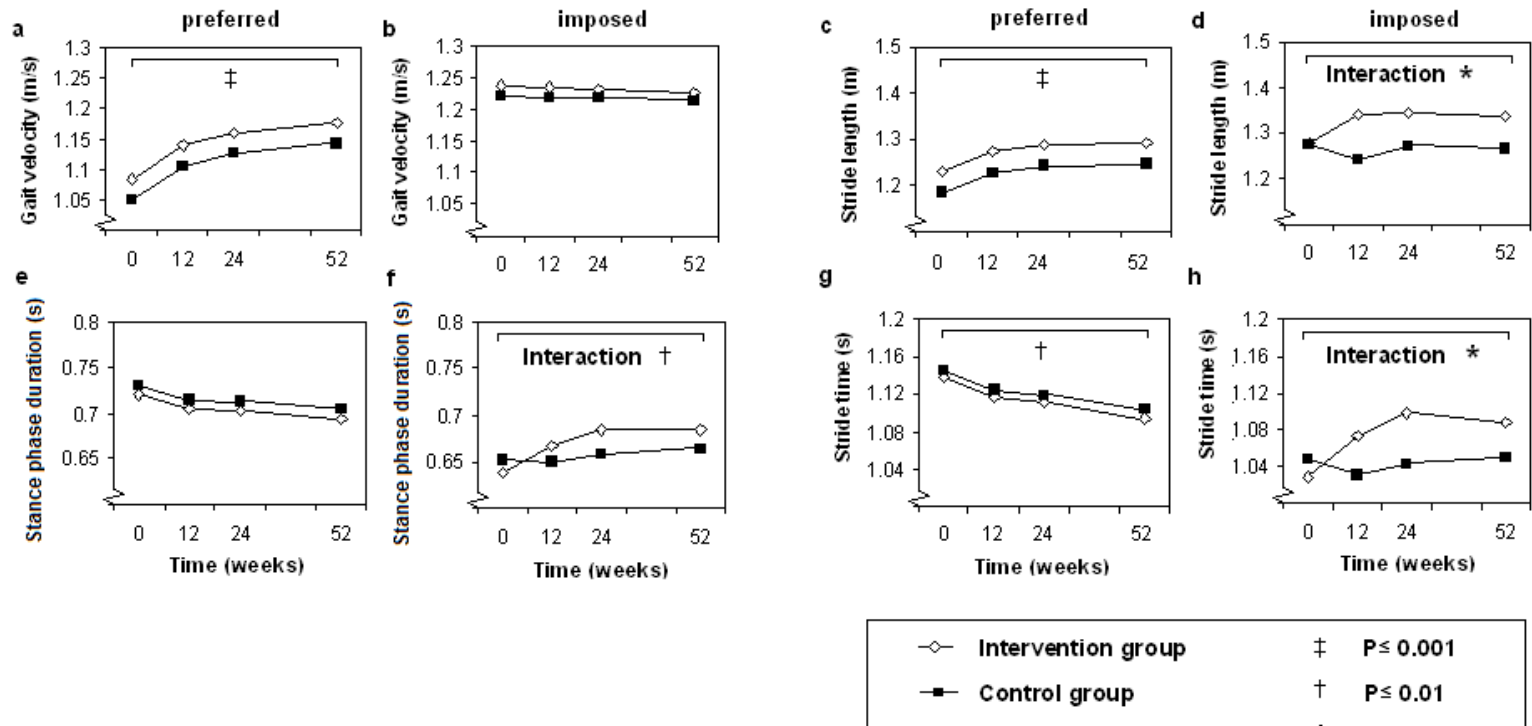

$\begin{array}{ll}\ddagger & \mathbf{P} \leq 0.001 \\ \dagger & \mathbf{P} \leq 0.01 \\ * & \mathbf{P} \leq 0.05\end{array}$

Figure 2(a-h). Estimated mean values of spatiotemporal gait characteristics over time for intervention and control groups, according to the final random intercept models (table 3). Significant time effects over 52 weeks and interaction effects are marked with: $* p \leq 0.05,+p \leq 0.01, \neq p \leq 0.001$. 
Table 3(a-n). The determinants and their effects on gait dynamics in diabetic patients with polyneuropathy depicted as $\hat{\beta}$ (CI 95).

\begin{tabular}{|c|c|c|c|c|}
\hline & $\begin{array}{r}\text { Preferred } \\
\text { a. H-ext }(\mathbf{N} . \mathbf{m} / \mathbf{k g})\end{array}$ & $\begin{array}{r}\text { Imposed } \\
\text { b. H-ext ( } \mathbf{N} \cdot \mathbf{m} / \mathbf{k g})\end{array}$ & $\begin{array}{r}\text { Preferred } \\
\text { C. H-flex }(\mathbf{N} . \mathbf{m} / \mathbf{k g}) \\
\end{array}$ & $\begin{array}{r}\text { Imposed } \\
\text { d. H-flex (N.m/kg) }\end{array}$ \\
\hline Intercept & $.543(.125, .961)^{*}$ & $.395(-.067, .816)$ & $1.053(-1.592,-$ & $.774(-1.409,-.139)^{*}$ \\
\hline Age (years) & $.003(-.003, .009)$ & $.006(-.001, .013)$ & $-.006(-.002, .013)$ & $-.002(-.007, .011)$ \\
\hline Gender (F/ M) & $.041(-.070, .151)$ & $.031(-.080, .142)$ & $-.036(-.109, .180)$ & $.071(-.237, .094)$ \\
\hline \multicolumn{5}{|l|}{ Body mass (kg) } \\
\hline Group (IG vs CG) & $-.031(-.116, .054)$ & $-.047(-.136, .042)$ & $-.070(-.039, .179)$ & $-.018(-.109, .145)$ \\
\hline Time $t=0$ vs 52 & $-.097(-.170,-.025) \dagger$ & $-.034(-.110, .041)$ & $-.112(.033, .190) \dagger$ & $-.055(-.026, .136)$ \\
\hline Time $t=12$ vs 52 & $-.037(-.113, .039)$ & $-.026(-.104, .053)$ & $-.093(.012, .174)^{*}$ & $-.119(.037, .201) \dagger$ \\
\hline Time $t=24$ vs 52 & $.034(-.045, .113)$ & $.006(-.074, .086)$ & $.005(-.089, .079)$ & $.035(-.117, .048)$ \\
\hline$\sigma_{i}^{2}$ & $.033(.026, .043) \neq$ & $.030(.023, .040) \neq$ & $.038(.029, .048) \neq$ & $.032(.024, .042)$ \\
\hline \multirow[t]{2}{*}{$\sigma_{b_{0}}^{2}$} & $.024(.014, .042) \neq$ & $.023(.012, .042) \neq$ & $.050(.033, .075) \neq$ & $.068(.045, .102)$ \\
\hline & e. K-ext (N.m/kg) & f. K-ext (N.m/kg) & g. K-flex (N.m/kg) & h. K-flex (N.m/kg) \\
\hline Intercept & $.655(-1.013,-.297)^{\dagger}$ & $.513(-.910,-.115)^{*}$ & $.503(.126, .879) \dagger$ & $.698(.227,1.169) \dagger$ \\
\hline Age (years) & $-.001(-.005, .006)$ & $.002(-.008, .004)$ & $-.006(-.012,-.001)^{*}$ & $-.005(-.011, .001)$ \\
\hline Gender (F/ M) & $-.044(-.052, .140)$ & $.025(-.078, .128)$ & $.021(-.082, .124)$ & $.067(-.050, .184)$ \\
\hline Body mass (kg) & & & & $-.003(-.006,-.001)^{*}$ \\
\hline Group (IG vs CG) & $.002(-.074, .071)$ & $-.009(-.070, .089)$ & $.039(-.036, .115)$ & $.012(-.067, .092)$ \\
\hline Time $t=0$ vs 52 & $-.149(.096, .202) \neq$ & $-.102(.051, .154) \neq$ & $.089(.045, .134) \neq$ & $.109(.060, .158) \neq$ \\
\hline Time $t=12$ vs 52 & $-.057(.003, .112)^{*}$ & $-.025(-.027, .078)$ & $.037(-.009, .082)$ & $.035(-.015, .085)$ \\
\hline Time $t=24$ vs 52 & $-.015(-.041, .072)$ & $-.017(-.036, .070)$ & $.003(-.044, .050)$ & $-.007(-.057, .043)$ \\
\hline$\sigma_{i}^{2}$ & $.017(.013, .022) \neq$ & $.013(.010, .017)^{\ddagger}$ & $.012(.009, .015) \neq$ & $.012(.009, .015) \neq$ \\
\hline \multirow[t]{2}{*}{$\sigma_{b_{0}}^{2}$} & $.022(.014, .033) \neq$ & $.026(.017,0.39)^{\ddagger}$ & $.029(.020, .042) \neq$ & $.027(.018, .041) \neq$ \\
\hline & i. A-40 (N.m/kg) & j A-40 (N.m/kg) & k. A-plant & I. A-plant \\
\hline Intercept & $.4777(.127, .828)^{\dagger}$ & $.406(.020, .791)^{*}$ & 2.359 & 2.439 \\
\hline Age (years) & $.004(-.001, .009)$ & $.005(-.001, .011)$ & $-.009(-.013,-.004) \neq$ & $-.010(-.015,-.005) \neq$ \\
\hline Gender (F/ M) & $.066(-.030, .161)$ & $.083(-.017, .183)$ & $.115(.021, .208) \dagger$ & $.119(.015, .222)^{*}$ \\
\hline Body mass (kg) & & & $-.003(-.005,-.001) \dagger$ & $-.003(-.006,-.001) \dagger$ \\
\hline Group (IG vs CG) & $-.006(-.076, .065)$ & $.027(-.051, .104)$ & $.038(-.024, .101)$ & $.020(-.052, .092)$ \\
\hline Time $t=0$ vs 52 & $-.062(-.105,-.018) \dagger$ & $-.053(-.104,-.003)$ & $-.030(-.077, .016)$ & $-.015(-.068, .037)$ \\
\hline Time $t=12$ vs 52 & $-.029(-.073, .015)$ & $-.031(-.082, .020)$ & $.003(-.048, .048)$ & $.005(-.048, .059)$ \\
\hline Time $t=24$ vs 52 & $-.027(-.073, .019)$ & $-.007(-.059, .045)$ & $.016(-.034, .066)$ & $.018(-.036, .072)$ \\
\hline$\sigma_{i}^{2}$ & $.011(.009, .014)^{\ddagger}$ & $.012(.009, .016) \neq$ & $.013(.010, .017) \neq$ & $.014(.010, .018) \neq$ \\
\hline \multirow[t]{2}{*}{$\sigma_{b_{0}}^{2}$} & $.024(.017, .035) \neq$ & $.024(.016, .037)^{\ddagger}$ & $.015(.010, .024) \neq$ & $.019(.012, .030)^{\ddagger}$ \\
\hline & m. A-40/ A-Plant & n. A-40/ A-Plant & & \\
\hline Intercept & $.160(-.066, .387)$ & $.105(-.161, .372)$ & & \\
\hline Age (years) & $.005(.002, .008) \dagger$ & $.006(.002, .010) \dagger$ & & \\
\hline Gender (F/ M) & $.029(-.033, .091)$ & $.039(-.031, .109)$ & & \\
\hline \multicolumn{5}{|l|}{ Body mass (kg) } \\
\hline Group (IG vs CG) & $-.020(-.066, .025)$ & $.005(-.048, .059)$ & & \\
\hline Time $t=0$ vs 52 & $-.032(-.059,-.005)$ & $-.031(-.063, .000)$ & & \\
\hline Time $t=12$ vs 52 & $-.018(-.045, .010)$ & $-.020(-.052, .013)$ & & \\
\hline Time $t=24$ vs 52 & $-.020(-.049, .008)$ & $-.009(-.041, .023)$ & & \\
\hline$\sigma_{i}^{2}$ & $.004(.003, .005) \neq$ & $.005(.004, .006) \neq$ & & \\
\hline$\sigma_{b_{0}}^{2}$ & $.010(.007, .015) \neq$ & $.013(.008, .019) \neq$ & & \\
\hline
\end{tabular}

Note. Significant differences are marked with: $* p \leq 0.05 ;+p \leq 0.01 ; \neq p \leq 0.001$. Abbreviations: IG= intervention group; $C G=$ control group; $M=$ male; F= Female.

The maximal knee joint flexion moment was lower for older participants, every extra year of age at onset of the study concurred with a $3.2 \%$ significantly smaller maximal knee joint flexion moment during the preferred gait velocity $\left(0.006 \mathrm{~N} . \mathrm{m}^{\mathrm{kg}}{ }^{-1}, p=0.02\right.$, figure and table $\left.3 \mathrm{~g}\right)$. A higher age at onset of the study was associated with a reduction of A-plant of $0.6 \%\left(0.009 \mathrm{~N} . \mathrm{m}^{\mathrm{kg}} \mathrm{kg}^{-1}, p<0.001\right.$, figure/ table $3 \mathrm{k})$ and $0.7 \%\left(0.010 \mathrm{~N} \cdot \mathrm{m} . \mathrm{kg}^{-1}, p<0.001\right.$, figure and table $\left.3 \mathrm{l}\right)$ per year for the preferred and imposed gait velocity respectively. Consequently A-40/A-plant increased with aging 
also with $1.0 \%$ at the preferred $(0.005, p \leq 0.01$, figure and table $3 \mathrm{~m})$ and $1.3 \%$ at the imposed gait velocity $(0.006, p \leq 0.01$, figure and table $3 n)$. The preferred gait velocity decreased with $0.7 \%$ (0.008 m.s $\mathrm{m}^{-1}, p<0.001$, figure and table 2a), stride length decreased with $0.5 \%(0.006 \mathrm{~m}, p<$ 0.001 , figure and table 2c) and stance phase duration and stride time both increased with $0.4 \%$ ( 0.003 and $0.004 \mathrm{~s}$ respectively, $p \leq 0.01$, figure and table $2 \mathrm{e}$ and $\mathrm{g}$ ) for every extra year of age.

People with a higher body mass experienced a proportionally lower, maximal knee joint flexion moment during the imposed gait velocity of $1.5 \%$ per $\mathrm{kg}$ of body mass $\left(0.003 \mathrm{~N} . \mathrm{m} . \mathrm{kg}^{-1}, p \leq\right.$ 0.01 , figure and table $3 \mathrm{~h}$ ) and proportionally lower maximum ankle plantar flexion moments of $0.2 \%$ per $\mathrm{kg}$ of body mass at both gait velocities $\left(0.003 \mathrm{~N} . \mathrm{m} . \mathrm{kg}^{-1}, p \leq 0.01\right.$, figure and table $3 \mathrm{k}$ and I).

Corrected for body mass, the women in this study had a higher maximum ankle plantar flexion moment compared to the men of 8.0 and $7.5 \%$ for the preferred and imposed gait velocity respectively (0.115 N.m. $\mathrm{kg}^{-1}, p=0.02 ; 0.119 \mathrm{~N} . \mathrm{m} . \mathrm{kg}^{-1}, p=0.03$, figure and table $3 \mathrm{k}$ an I).
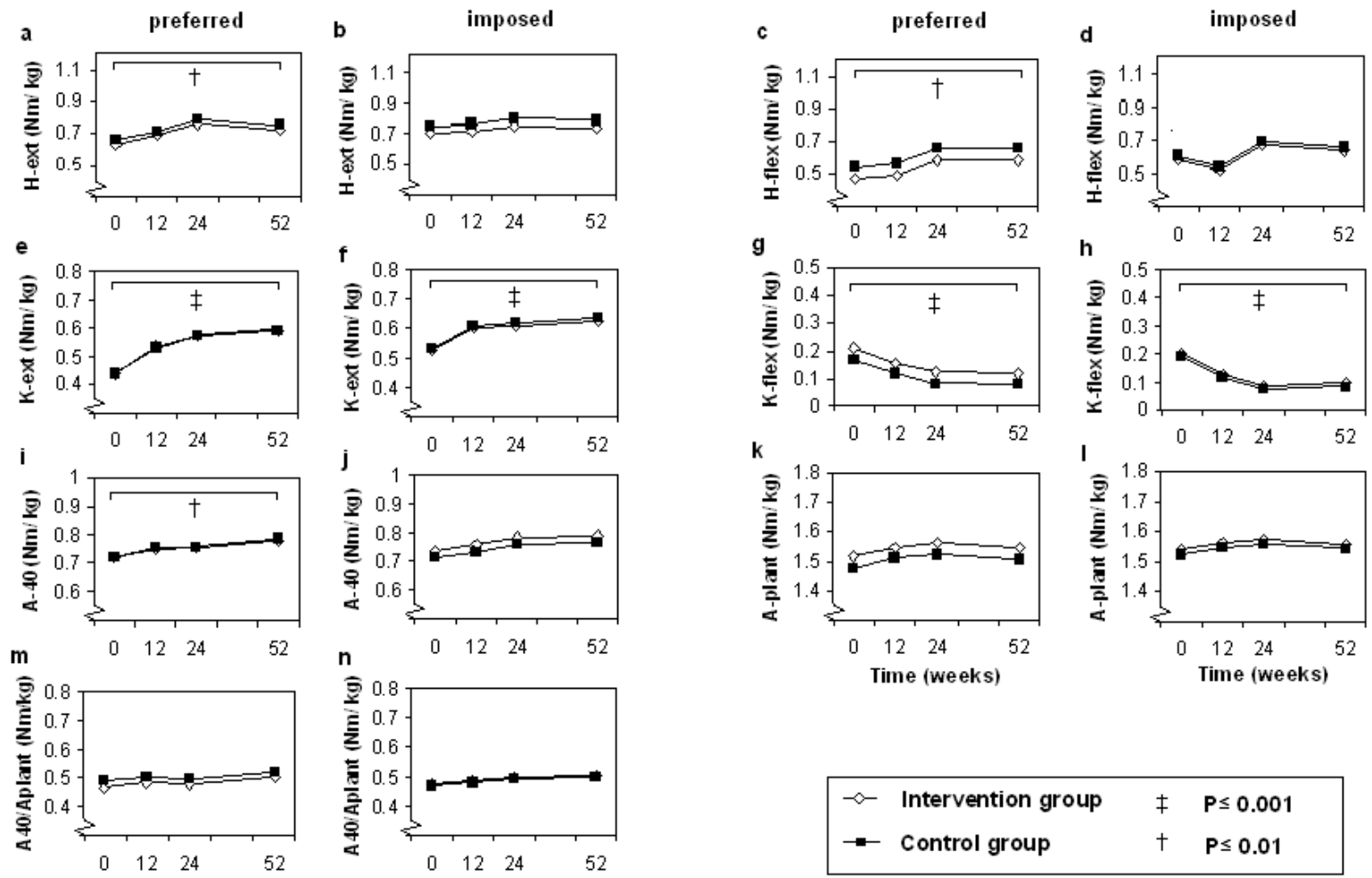

Figure 3(a-n). Estimated mean values of gait dynamics over time for intervention and control groups, according to the final random intercept models (table 2). Abbreviations: $\mathrm{H}$-ext: maximal hip extension; $\mathrm{H}$-flex: maximal hip flexion; K-flex: maximal knee flexion; K-ext: maximal knee extension. A-40: Plantar flexion moment at $40 \%$ of the stance phase; A-plant: Maximal plantar flexion moment. Significant time effects over 52 weeks and group differences are marked with: * $p \leq 0.05,+p \leq 0.01, \neq p \leq 0.001$.

\section{Discussion}

The aim of the current study was to evaluate the effect of strength training on lower extremity joint moments and spatiotemporal gait characteristics in patients with DPN. We hypothesized that improving lower extremity muscle strength would enable a better control of the roll-off of the foot, a slower forward displacement of the point of application of the GRF and consequently a lower 
ankle plantar flexion moment during the first part of the stance phase and a higher knee extension moment, but the training did not influence joint moments in our patients and consequently it is to be expected that forefoot loading during a step was neither affected. However, the effect of the training could have been underestimated, as stride length increased, as discussed below. A larger stride length leads to a higher ankle plantar flexion moment which could have negated an effect of training on lowering the ankle plantar flexion moment [7]. In addition, the intensity of the training program could have been too low to improve joint moments during gait. To be reported elsewhere, the training program did not increase maximal dorsal flexor strength, but did increase maximum plantar flexion strength (IJzerman et al., unpublished data). However, these data suggest that an increase in maximum plantar flexion did not influence the forces generated during gait. Alternatively, the lack of effect of the intervention on joint moments and maximum dorsal flection strength may have been caused by nerve damage so severe that a positive effect of a muscle strengthening program was unattainable. It is also possible that the altered control of the roll-off of the foot in diabetic neuropathy does not so much depend on the result of loss of muscle strength, but more on other factors, such us joint motion, motor control, feedback from muscle spindles, rate of force development and sensory nerve function of the skin $[4,8,14,15]$. The number of studies on the underlying mechanisms of gait alteration in DPN and especially on strength training in this population is to our knowledge very limited and our study can be one of the building blocks for future intervention studies in these patients.

To our knowledge, there is one published study on the effects of exercise on spatiotemporal gait characteristics in DPN. In this study [16] gait and balance exercises in DPN participants resulted in longer strides, a shorter stride time and shorter stance phase duration measured during preferred gait velocity. We did not observe an effect of training at the preferred gait velocity, as both the intervention and control group increased their gait velocity over time. The training did however result in a longer stride length, stride time and stance phase duration during the imposed gait velocity, which is in part in line with the aforementioned study [16]. If and to which extent these changes in spatiotemporal gait characteristics result in subjective improvement of walking quality and a more stable walking pattern needs to be determined in future studies.

Over a period of 52 weeks we observed several changes in joint moments in both groups which could at least in part be explained by changes in gait velocity. During the preferred gait velocity, the joint moments for hip extension and flexion, knee extension and ankle plantar flexion at $40 \%$ of the stance phase all increased. As shown earlier in healthy young adults, hip extension and flexion moments increase with a faster gait velocity [17]. Using the equations of this aforementioned study, more than $50 \%$ of the changes in joint moments in our participants could be explained by the increase in gait velocity. In addition, our participants also had an increase of knee extension moment and a decrease of knee flexion moment over time, which is difficult to explain based on the current data. More detailed studies are needed to explore the mechanisms underlying these changes over time and in particular to what extent these changes are related to progression of the underlying neuropathy.

In addition to effects of the training on spatiotemporal gait characteristics, we also observed a time-dependent effect, independent of the training. Longitudinal data on the walking pattern in DPN patients are scarce. Over the period of 52 weeks, participants of both the intervention and control group preferred to walk faster. Also other authors noted an increase in 
preferred gait velocity regardless of an intervention $[18,19]$. It is likely that participants walking at a gait track for the first time decrease their preferred gait velocity due to unfamiliarity with the test settings. Peripheral sensory loss due to DPN could further contribute to this less confident gait pattern [20] and the more often participants are measured the more familiar they become with the test track, resulting in a larger stride length and an increased gait velocity. Our patients with a slow preferred gait velocity seem to be more often in a poorer health condition and therefore more vulnerable, possibly contributing to a higher likelihood of dropping out. Consequently, the increase of gait velocity in our patients over time could be the result of selective drop-out.

The current study clearly has some limitations; the attrition rate was high for both the intervention and control group and the pattern of missingness in the statistical analyses indicates that participants measured were not per definition a random selection of the group. The high dropout rate is a known problem for long term exercise programs in patients with diabetes, e.g. a dropout rate of $60 \%$ was observed during brisk walking program [21-23]. In the current study the problem of missingness is, at least partly, circumvented by the application of the mixed model. Nevertheless, statistical significant differences need to be considered with caution. Furthermore, we did not correct for multiple testing, but several spatiotemporal gait characteristics changed in parallel, suggesting that gait characteristics can be influenced by training.

The current study provides valuable information for future longitudinal studies on strength training and gait analysis in participants with DPN. The study showed that these patients have a high risk for drop-out, due to the underlying pathology and co-morbidities. Hence, future studies should preferably first focus on participants with a relatively good health status and early neuropathy. The highest drop-out rate was observed during the first 12 weeks of the program in both the intervention and control group and several participants reported lack of motivation as reason for dropping-out. Lack of physical activity is an important factor in the development of type 2 diabetes and its complications [24,25]. Future studies should focus on exploring the effect of motivational factors on exercise compliance in DPN, delineating these factors and find new ways to coach our patients in improving their mobility. However, it should be noted that strict selection of participants also has a draw-back, as results might be less generalizable to the population of DPN patients. Secondly, as in the current study 16 patients were not able to safely walk over the test track at $1.2 \mathrm{~m} / \mathrm{s}$, future studies on gait in DPN patients might consider a slower standardized gait velocity. Finally, the increase of gait velocity over time should be accounted for in future studies, given the importance of gait velocity for joint moments or spatiotemporal gait characteristics.

It is concluded that strength training did not affect the maximal amplitude of hip, knee and ankle joint moments, and did increase stride length, stride time and stance phase duration in patients with DPN walking at a standardized, imposed, gait velocity, but the underlying mechanisms are unclear. More specified research exploring the effects of similar or other interventions is necessary. Future studies should focus on defining the motivational and health status related factors that will affect the outcome of the clinical trials on the effect of these training programs before their feasibility and effectiveness can be tested in patients with DPN. 


\section{References}

1. Caselli A, Pham H, Giurini JM, Armstrong DG, Veves A: The forefoot-to-rearfoot plantar pressure ratio is increased in severe diabetic neuropathy and can predict foot ulceration. Diabetes Care 2002, 25(6):1066-1071.

2. Abboud RJ, Rowley DI, Newton RW: Lower limb muscle dysfunction may contribute to foot ulceration in diabetic patients. Clin Biomech (Bristol, Avon) 2000, 15(1):37-45.

3. Kwon OY, Minor SD, Maluf KS, Mueller MJ: Comparison of muscle activity during walking in subjects with and without diabetic neuropathy. Gait Posture 2003, 18(1):105-113.

4. Savelberg HH, Ilgin D, Angin S, Willems PJ, Schaper NC, Meijer K: Prolonged activity of knee extensors and dorsal flexors is associated with adaptations in gait in diabetes and diabetic polyneuropathy. Clin Biomech (Bristol, Avon) 2010, 25(5):468-475.

5. Savelberg HH, Schaper NC, Willems PJ, de Lange TL, Meijer K: Redistribution of joint moments is associated with changed plantar pressure in diabetic polyneuropathy. BMC Musculoskelet Disord 2009, $10: 16$

6. Melai T, Schaper NC, IJzerman TH, De Lange ALH, Willems PJB, Meijer K, Lieverse A.G., Savelberg HHCM: Increased forefoot loading is associated with an increased plantar flexion moment. Human movement Science 2013, in press.

7. Allet L, H IJ, Meijer K, Willems P, Savelberg H: The influence of stride-length on plantar foot-pressures and joint moments. Gait Posture 2011, 34(3):300-306.

8. Courtemanche R, Teasdale N, Boucher P, Fleury M, Lajoie $Y$, Bard C: Gait problems in diabetic neuropathic patients. Arch Phys Med Rehabil 1996, 77(9):849-855.

9. Mueller MJ, Minor SD, Sahrmann SA, Schaaf JA, Strube MJ: Differences in the gait characteristics of patients with diabetes and peripheral neuropathy compared with age-matched controls. Phys Ther 1994, 74(4):299-308; discussion 309-213.

10. Valk GD, de Sonnaville JJ, van Houtum WH, Heine RJ, van Eijk JT, Bouter LM, Bertelsmann FW: The assessment of diabetic polyneuropathy in daily clinical practice: reproducibility and validity of Semmes Weinstein monofilaments examination and clinical neurological examination. Muscle Nerve 1997, 20(1):116-118.

11. Chetlin RD, Gutmann L, Tarnopolsky M, Ullrich IH, Yeater RA. Resistance training effectiveness in patients with Charcot-Marie-Tooth disease: recommendations for exercise prescription. Arch Phys Med Rehabil 2004, 85(8):1217-1223.

12. Andersen $\mathrm{H}$, Schmitz $\mathrm{O}$, Nielsen $\mathrm{S}$. Decreased isometric muscle strength after acute hyperglycaemia in Type 1 diabetic patients. Diabet Med 2005, 22(10):1401-1407.

13. Moisio KC, Sumner DR, Shott S, Hurwitz DE. Normalization of joint moments during gait: a comparison of two techniques. J Biomech 2003, 36(4):599-603.

14. Delbridge L, Perry $P$, Marr $S$, et al. Limited joint mobility in the diabetic foot: relationship to neuropathic ulceration. Diabet Med 1988, 5(4):333-337.

15. van Deursen RW, Sanchez MM, Ulbrecht JS, Cavanagh PR. The role of muscle spindles in ankle movement perception in human subjects with diabetic neuropathy. Exp Brain Res 1998, 120(1):1-8.

16. Allet L, Armand S, Aminian K, Pataky Z, Golay A, de Bie RA, de Bruin ED: An exercise intervention to improve diabetic patients' gait in a real-life environment. Gait Posture 2010, 32(2):185-190.

17. Lelas JL, Merriman GJ, Riley PO, Kerrigan DC: Predicting peak kinematic and kinetic parameters from gait speed. Gait Posture 2003, 17(2):106-112.

18. Tsang T, Orr R, Lam P, Comino EJ, Singh MF: Health benefits of Tai Chi for older patients with type 2 diabetes: the "Move It For Diabetes study"--a randomized controlled trial. Clin Interv Aging 2007, 2(3):429-439.

19. Orr R, Tsang T, Lam P, Comino E, Singh MF: Mobility impairment in type 2 diabetes: association with muscle power and effect of Tai Chi intervention. Diabetes Care 2006, 29(9):2120-2122.

20. Menz HB, Lord SR, St George R, Fitzpatrick RC: Walking stability and sensorimotor function in older people with diabetic peripheral neuropathy. Arch Phys Med Rehabil 2004, 85(2):245-252.

21. Monteiro MA, Gabriel RE, Neves ECM, Sousa MF, Abrantes JM, Moreira MH. Exercise effects in plantar pressure of postmenopausal women. Menopause 2010: 17(5):1017-1025.

22. Praet SF, van Rooij ES, Wijtvliet A, et al. Brisk walking compared with an individualised medical fitness programme for patients with type 2 diabetes: a randomised controlled trial. Diabetologia 2008: 51(5):736-746.

23. Thoolen B, de Ridder D, Bensing J, Gorter K, Rutten G. Who participates in diabetes self-management interventions?: Issues of recruitment and retainment. Diabetes Educ 2007: 33(3):465-474.

24. Gill JM, Cooper AR: Physical activity and prevention of type 2 diabetes mellitus. Sports Med 2008, 38(10):807-824.

25. Hu FB, Leitzmann MF, Stampfer MJ, Colditz GA, Willett WC, Rimm EB: Physical activity and television watching in relation to risk for type 2 diabetes mellitus in men. Archives of internal medicine 2001, 161(12): 1542-1548. 


\section{Chapter 7}

Summary and general discussion 


\section{Introduction}

High plantar pressures are an important risk factor for plantar ulceration in diabetic polyneuropathy (DPN) and as plantar pressures are increased in DPN [1,2], it is important to understand the origin of these higher pressures. The series of events in the lower leg and foot leading from neuropathy to increased loading of the plantar area is however not yet fully understood. Therefore, the aim of the current dissertation is to contribute to the body of knowledge on the etiology of high plantar pressures in DPN by providing more insight in the gait related mechanisms responsible for increased plantar pressures in this population. A second aim is to evaluate the effects of a specific physical therapy exercise program developed to improve the gait related factors that were considered to be responsible for increased plantar pressures in DPN.

\section{Main findings and discussion}

Gait related determinants of plantar pressure in DPN.

Chapter 2 describes a literature review on the gait related mechanisms responsible for increased plantar pressures in DPN, which points to involvement of the activation of plantar and dorsal flexors in the development of high plantar pressures in DPN [3-9]. Also limitations in the control of the ground reaction forces and joint moments of the lower extremity have a considerable influence [10-15], but the exact cascade through which plantar pressures are affected is still unclear. In DPN spatiotemporal gait characteristics such as gait velocity and stride length are affected $[8,13,14$, 16-19], can have a large effect on plantar pressures [20-22] and should therefore be monitored or standardized in future studies on plantar pressure measurements. There is some evidence that changing gait characteristics in DPN can be used to lower plantar pressures and thereby to decrease the risk for ulceration [5, 20-23]. In addition, another approach, such as using walking aids could also be useful to increase daily activity [24], without increasing the risk of excessive plantar loading. In order to prevent high forefoot loading it is also important to avoid muscle fatigue $[25,26]$ and patients with a high risk for ulceration should not take long walks and divide the time spent walking over the day. The review in chapter 2 confirmed that gait related factors play an important role in the development of high plantar pressures, which stresses the importance for clinicians to look further than passive shoe and offloading therapy in the prevention or treatment of increased plantar loading in DPN or other pathology.

The pressure-time integral as measure for plantar loading.

Peak pressure is the most commonly used plantar pressure variable to assess the effects of DPN on plantar loading. Another variable is the pressure-time integral (PTI), which describes the cumulative effects of pressure over time in a certain area of the foot, and thus provides a value for the total load exposure of a foot sole area during one step [27]. As there is a high correlation between peak pressure and the PTI calculated by Novel software, the relevance of reporting both peak pressure and PTI might be questioned [28]. But, the software most commonly used to assess the PTI (Novel GmbH Inc., Munich, Germany), does not represent an integral over time, as it summates the products of peak pressures per time sample and the duration of that sample. In 
chapter 3, the quotient of the force-time integral over contact area was obtained as an alternative calculation of the PTI. This new alternative method correlated less with peak pressure than the PTI as calculated by Novel. The two methods differed significantly and these differences varied between foot sole areas and between groups. In the group with diabetic polyneuropathy, the largest differences were found under the metatarsal heads, an area particularly prone to recurrent ulceration. From a theoretical perspective, the alternative approach provides a more valid calculation of the PTI. In addition, we showed that the alternative calculation is of added value, alongside peak pressure calculation, to interpret altered plantar pressures patterns in particular in patients at risk for foot ulceration.

The link between plantar pressures and joint moments.

To obtain more insight in the underlying gait related mechanisms that could contribute to adverse plantar pressures, an instrumented gait analysis was performed in combination with plantar pressure measurement in DPN subjects, which is described in chapter 4. During baseline measurements of the randomized controlled trial that will be discussed below, a series of correlations was observed connecting an increase in ankle joint moment to an increase in forefoot loading. The data suggest that people with a relatively high plantar flexion joint moment at $40 \%$ of the stance phase, have a faster forward transfer of center of pressure and consequently higher loading of the forefoot. Interventions that are aimed at increasing the control of the roll-off of the foot could therefore contribute to a better distribution of plantar pressures. Besides shoe and offloading technology, also physiotherapeutic interventions such as strength and gait training might contribute to the prevention of high plantar pressures.

\section{Therapy for increased forefoot loading in DPN.}

Chapter 5 describes a study embedded in a randomized controlled trial, in which the effect of a specific physical therapy exercise program to diminish high plantar pressures of people with DPN was evaluated. During 24 weeks the intervention group received one group session per week, which consisted of strength training of the lower extremity muscles. The intervention group was also asked to perform home exercises two times a week. The control group received no intervention. Plantar pressures were measured before, during, directly after and at follow-up of the intervention, at respectively $0,12,24$ and 52 weeks of the start of the program. All measurements were performed at a preferred and a standardized, imposed gait velocity. No effect of the intervention on plantar pressures could be observed over a period of 52 weeks, but we did observe that both peak pressure and PTI increased over this time period in both the intervention and control group, resulting in a progressive rise in the risk of foot ulceration. The pathways leading to this rise are not yet fully clear and demand a better understanding of the etiology of these high pressures. Even more, the rapid increase of the risk for ulceration indicates the importance of interventions aimed at preventing or treating plantar pressures.

In chapter 6 we described the effects of the previously described exercise program on spatiotemporal gait characteristics and joint moments, because high forefoot loading can be caused by alterations in gait patterns due to DPN. Also these measurements were performed at a preferred and standardized imposed gait velocity. We did not observe any effects of the exercise program on 
the lower extremity joint moments during gait, as the maximal amplitude of hip, knee and ankle joint moments remained the same at both velocities. At the imposed gait velocity, the exercise program did result in an increase in stance phase duration of $5.5 \%$, stride time of $5.6 \%$ and stride length of $5.1 \%$. The cause of these alterations is not yet understood, but could be due to an increase in motor control. In both the intervention and control group, we observed an increase of the preferred gait velocity over the period of 52 weeks. This increase is in probably in part caused by a learning effect with the patient being better familiarized with the test settings.

\section{Limitations}

The research described in this dissertation has several limitations. For the alternative calculation of the PTI in a certain foot region the total contact area of that region is used, while the instantaneous extent of contact area may vary over time. However, this is only a theoretical limitation, because for the major part of the contact time of a foot sole area, most sensors of a specific area are loaded.

Another limitation was that the described research was embedded in a larger randomized controlled trial, of which the primary focus was to evaluate the effects of a physical therapy program on muscle strength and mobility of DPN patients. Therefore, to ensure the generalizability of the described research, more studies need to be performed on the same outcome measures.

A major limitation of the research described in this dissertation was the high drop-out rate during the randomized controlled trial and the highest rate was observed during the first 12 weeks of the training program. Gait related problems were a major reason for drop-out in one third of the patients who withdrew from the study and in addition the staff had the impression that lack of motivation also played an important role in several patients. The motivation to exercise is probably suboptimal in many diabetic patients as persons that are less motivated to exercise throughout their lives have a higher chance to develop diabetes and its complications [29, 30]. A high drop-out rate was also a major problem in other long term exercise programs in diabetic patients, e.g. a drop-out rate of $60 \%$ was observed during brisk walking program [31-33]. The problem of missingness in our research is at least partly circumvented by the application of the mixed model. Nevertheless, statistical significant differences on the effects of the intervention should be considered with caution. But, the effects observed changed in parallel with each other, suggesting that spatiotemporal gait characteristics can be influenced by training.

\section{Clinical implications, recommendations and future research}

Most researchers and clinicians currently focus on peak pressure to assess plantar loading and the risk of plantar tissue damage. However, there is evidence that also submaximal pressures can result in tissue damage $[34,35]$ and repetitive exposure to these submaximal pressures could inhibit regeneration processes, leading to cumulative lowering of tissue resistance. The peak pressure remains the most important variable, but to correctly assess the effects of plantar pressures on tissue damage, it is important to include a measure for cumulative pressures as well. The alternative calculation of the PTI as proposed and evaluated in chapter 3 provides a measure of 
cumulative loading of both maximal and submaximal pressures and can therefore be used as additional variable to assess plantar loading alongside peak pressure.

The individual and combined relation of peak pressure and the PTI to plantar tissue damage is not well defined and demands further research. A prospective study by Lavery et al. [36] provided more insight on the contribution of peak pressure to plantar tissue damage by evaluating patients that developed an ulcer two years after plantar pressure measurements, but this study did not report on the PTI. A comparable study, including both peak pressure and the PTI, could shed more light on how these variables are related to tissue damage. In addition, measuring temperature and plantar pressure variables during gait could also provide more insight on the effects of plantar pressures on tissue damage, as any form of tissue damage will result in an inflammatory process, which will lead to thermal reactions. Nevertheless, at this moment it can be concluded that to avoid plantar tissue damage it is important to avoid high peak pressures and PTIs.

When measuring plantar pressures it is important to realize that the outcome can be affected by several factors. Gait velocity is the most important factor and can be decreased in the presence of DPN, which will result in lower peak pressures [5, 21], but an increase of the PTI [5, 22]. Unfamiliarity with test settings can also lead to a lower gait velocity, and can consequently result in an underestimation of the peak pressures during gait outside test settings. When evaluating the effect of therapy on plantar pressures, it is therefore advisable to standardize gait velocity in at least one set of measurements. Other spatiotemporal gait characteristics, such as stride length can also affect plantar pressure pressures, as a shorter stride length will lead to a lower peak pressure and PTI [37]. To render measurements comparable it is important to standardize or monitor gait velocity and stride length during plantar pressure measurements.

The rapid increase of plantar pressures in patients with DPN suggests an increased risk for plantar ulceration, due to the progressive nature of diabetic polyneuropathy. This finding can be used for interpretation and design of future longitudinal studies on plantar pressures. In addition, the rapid increase shows the importance of regularly monitoring subjects at risk for ulceration as well as the importance of the development of interventions to prevent a further increase.

Current treatment interventions consist mainly of offloading technology [38], such as shoe and insole adaptations, by means of which areas that are at risk or have been ulcerated can be relieved from high pressures. As this type of treatment does not interact with the underlying mechanisms responsible for these high pressures, it only treats the symptoms. The current dissertation concludes that alterations in gait pattern due to diabetic polyneuropathy can have considerable effects on plantar pressures. An inability to generate enough force to brake the forward velocity during the first half of the stance phase contributes to a shift of pressure towards the forefoot, which suggests that muscle weakness, a limited rate of force development, or delayed muscle activation contributes to adverse forefoot loading. Interventions aimed at increasing the control of the role off of the foot may therefore contribute to a better distribution of plantar pressures.

In chapter 5 and 6 the effects of a specific strength training program on plantar pressures and underlying gait related factors was evaluated, but no effects were observed of this intervention on 
plantar pressures or force generating capacity over a period of 52 weeks. This lack of effect might be caused by impaired trainability of the subjects due to the underlying neuropathy or the possibility that increased forefoot loading in DPN may not originate from muscle weakness, but rather from abnormalities in other factors associated with joint motion, motor control, feedback from muscle spindles, rate of force development and sensory nerve function of the skin [8, 39-41]. It is also possible that the intensity of the training program or its effects on the plantar and dorsal flexors was too low. It is therefore possible that a more specified program with a higher intensity of training of the dorsal and plantar flexors could be more effective. Also motivational factors could have played an important role, as these factors might have led to a higher drop-out and less compliance. Therefore, for future interventions it is advised to monitor or control motivational factors as much as possible. It is also advisable to have at least two plenary sessions a week to ensure compliance. In addition, by adding tailored fit exercises and games these interventions can be made more fun making it easier for participants to adhere to the intervention.

In chapter 2 other interventions were described that might be used to lower peak pressures, such as reducing stride length or gait velocity, or using walking aids. These interventions could provide a strategy for DPN patients to increase their physical activity or mobility, without further increasing plantar pressures and the risk for ulceration. However, most of these strategies have been tested in healthy subjects and not in a population with DPN. To confirm the positive effects on plantar pressures in this population, it is important to test these strategies in subjects at risk for ulceration and preferably during activities of daily life using ambulant pressure measuring devices. In addition, if these strategies lead to lower plantar pressures in this population, then the next step would be to determine whether such newly learned gait strategies can be implemented in daily life. Another strategy that is expected to be effective is to avoid walking in bouts of long duration, as a decrease in motor control due to muscle fatigue can lead to increased forefoot loading $[25,26]$. The contribution of muscle fatigue to high plantar pressures can be further confirmed by exposing DPN to fatiguing exercises and measuring the effects on plantar pressures. Losing body mass will presumably result in lower plantar pressures and can therefore be beneficial for patients at risk for ulceration. But, as the contribution of body mass to plantar pressures is limited [42] and losing body mass is a challenging task, it is expected that this strategy is less feasible. 


\section{References}

1. Birke JA, Patout CA, Jr., Foto JG: Factors associated with ulceration and amputation in the neuropathic foot. J Orthop Sports Phys Ther 2000, 30(2):91-97.

2. Pitei DL, Lord M, Foster A, Wilson S, Watkins PJ, Edmonds ME: Plantar pressures are elevated in the neuroischemic and the neuropathic diabetic foot. Diabetes Care 1999, 22(12):1966-1970.

3. Ferris L, Sharkey NA, Smith TS, Matthews DK: Influence of extrinsic plantar flexors on forefoot loading during heel rise. Foot Ankle Int 1995, 16(8):464-473.

4. Akashi PM, Sacco IC, Watari R, Hennig E: The effect of diabetic neuropathy and previous foot ulceration in EMG and ground reaction forces during gait. Clin Biomech (Bristol, Avon) 2008, 23(5):584-592.

5. Warren GL, Maher RM, Higbie EJ: Temporal patterns of plantar pressures and lower-leg muscle activity during walking: effect of speed. Gait Posture 2004, 19(1):91-100.

6. Kwon OY, Minor SD, Maluf KS, Mueller MJ: Comparison of muscle activity during walking in subjects with and without diabetic neuropathy. Gait Posture 2003, 18(1):105-113.

7. Gomes AA, Onodera AN, Otuzi ME, Pripas D, Mezzarane RA, Sacco IC: Electromyography and kinematic changes of gait cycle at different cadences in diabetic neuropathic individuals. Muscle Nerve 2011, 44(2):258-268.

8. Savelberg HH, Ilgin D, Angin S, Willems PJ, Schaper NC, Meijer K: Prolonged activity of knee extensors and dorsal flexors is associated with adaptations in gait in diabetes and diabetic polyneuropathy. Clin Biomech (Bristol, Avon) 2010, 25(5):468-475.

9. Abboud RJ, Rowley DI, Newton RW: Lower limb muscle dysfunction may contribute to foot ulceration in diabetic patients. Clin Biomech (Bristol, Avon) 2000, 15(1):37-45.

10. Sacco IC, Amadio AC: A study of biomechanical parameters in gait analysis and sensitive cronaxie of diabetic neuropathic patients. Clin Biomech (Bristol, Avon) 2000, 15(3):196-202.

11. Uccioli L, Caselli A, Giacomozzi C, Macellari V, Giurato L, Lardieri L, Menzinger G: Pattern of abnormal tangential forces in the diabetic neuropathic foot. Clin Biomech (Bristol, Avon) 2001, 16(5):446-454.

12. Akashi PM, Sacco IC, Watari R, Hennig E: The effect of diabetic neuropathy and previous foot ulceration in EMG and ground reaction forces during gait. In: Clin Biomech (Bristol, Avon). vol. 23, 2008/01/08 edn; 2008: 584-592.

13. Shaw JE, van Schie $\mathrm{CH}$, Carrington $\mathrm{AL}$, Abbott $\mathrm{CA}$, Boulton $\mathrm{AJ}$ : An analysis of dynamic forces transmitted through the foot in diabetic neuropathy. Diabetes Care 1998, 21(11):1955-1959.

14. Yavuzer G, Yetkin I, Toruner FB, Koca N, Bolukbasi N: Gait deviations of patients with diabetes mellitus: looking beyond peripheral neuropathy. Eura Medicophys 2006, 42(2):127-133.

15. Mueller MJ, Minor SD, Sahrmann SA, Schaaf JA, Strube MJ: Differences in the gait characteristics of patients with diabetes and peripheral neuropathy compared with age-matched controls. Phys Ther 1994, 74(4):299-308; discussion 309-213.

16. Resnick HE, Stansberry KB, Harris TB, Tirivedi M, Smith K, Morgan P, Vinik AI: Diabetes, peripheral neuropathy, and old age disability. Muscle Nerve 2002, 25(1):43-50.

17. Sawacha Z, Gabriella G, Cristoferi G, Guiotto A, Avogaro A, Cobelli C: Diabetic gait and posture abnormalities: a biomechanical investigation through three dimensional gait analysis. Clin Biomech (Bristol, Avon) 2009, 24(9):722-728.

18. Petrofsky J, Lee S, Bweir S: Gait characteristics in people with type 2 diabetes mellitus. Eur J Appl Physiol 2005, 93(5-6):640-647.

19. Resnick HE, Vinik AI, Schwartz AV, Leveille SG, Brancati FL, Balfour J, Guralnik JM: Independent effects of peripheral nerve dysfunction on lower-extremity physical function in old age: the Women's Health and Aging Study. Diabetes Care 2000, 23(11):1642-1647.

20. Allet L, H IJ, Meijer K, Willems P, Savelberg H: The influence of stride-length on plantar foot-pressures and joint moments. In: Gait Posture. vol. 34, 2011/06/28 edn; 2011: 300-306.

21. Segal A, Rohr E, Orendurff M, Shofer J, O'Brien M, Sangeorzan B: The effect of walking speed on peak plantar pressure. Foot Ankle Int 2004, 25(12):926-933.

22. Burnfield JM, Few CD, Mohamed OS, Perry J: The influence of walking speed and footwear on plantar pressures in older adults. Clin Biomech (Bristol, Avon) 2004, 19(1):78-84.

23. Drerup B, Szczepaniak A, Wetz HH: Plantar pressure reduction in step-to gait: a biomechanical investigation and clinical feasibility study. Clin Biomech (Bristol, Avon) 2008, 23(8):1073-1079.

24. Perez-Soriano P, Llana-Belloch S, Martinez-Nova A, Morey-Klapsing G, Encarnacion-Martinez A: Nordic walking practice might improve plantar pressure distribution. Res Q Exerc Sport 2011, 82(4):593-599.

25. Stolwijk NM, Duysens J, Louwerens JW, Keijsers NL: Plantar pressure changes after long-distance walking. Med Sci Sports Exerc 2010, 42(12):2264-2272.

26. Bisiaux M, Moretto P: The effects of fatigue on plantar pressure distribution in walking. Gait Posture 2008, 28(4):693-698.

27. Sauseng S, Kastenbauer T, Sokol G, Irsigler K: Estimation of risk for plantar foot ulceration in diabetic patients with neuropathy. Diabetes Nutr Metab 1999, 12(3):189-193.

28. Waaijman R, Bus SA: The interdependency of peak pressure and pressure-time integral in pressure studies on diabetic footwear: no need to report both parameters. Gait Posture 2012, 35(1):1-5.

29. Gill JM, Cooper AR: Physical activity and prevention of type 2 diabetes mellitus. Sports Med 2008, 38(10):807-824.

30. Hu FB, Leitzmann MF, Stampfer MJ, Colditz GA, Willett WC, Rimm EB: Physical activity and television watching in relation to risk for type 2 diabetes mellitus in men. Archives of internal medicine 2001, 161(12):1542-1548. 
31. Praet SF, van Rooij ES, Wijtvliet A, Boonman-de Winter LJ, Enneking T, Kuipers H, Stehouwer CD, van Loon LJ: Brisk walking compared with an individualised medical fitness programme for patients with type 2 diabetes: a randomised controlled trial. Diabetologia 2008, 51(5):736-746.

32. Thoolen B, de Ridder D, Bensing J, Gorter K, Rutten G: Who participates in diabetes self-management interventions?: Issues of recruitment and retainment. Diabetes Educ 2007, 33(3):465-474.

33. Monteiro MA, Gabriel RE, Neves ECM, Sousa MF, Abrantes JM, Moreira MH: Exercise effects in plantar pressure of postmenopausal women. Menopause 2010,17(5):1017-1025.

34. Armstrong DG, Peters EJ, Athanasiou KA, Lavery LA: Is there a critical level of plantar foot pressure to identify patients at risk for neuropathic foot ulceration? J Foot Ankle Surg 1998, 37(4):303-307.

35. Birke JA, Novick, A., Hawkins, E.S., Patout jr, C.: A review of Causes of Foot Ulceration in Patients with Diabetes Mellitus. J Prosthet Orthot 1991, 4:9.

36. Lavery LA, Armstrong DG, Wunderlich RP, Tredwell J, Boulton AJ: Predictive value of foot pressure assessment as part of a population-based diabetes disease management program. Diabetes Care 2003, 26(4):1069-1073.

37. Allet $\mathrm{L}, \mathrm{H}$ IJ, Meijer $\mathrm{K}$, Willems $\mathrm{P}$, Savelberg $\mathrm{H}$ : The influence of stride-length on plantar foot-pressures and joint moments. Gait Posture 2011, 34(3):300-306.

38. Bus SA: Priorities in offloading the diabetic foot. Diabetes Metab Res Rev 2012, 28 Suppl 1:54-59.

39. Delbridge L, Perry P, Marr S, Arnold N, Yue DK, Turtle JR, Reeve TS: Limited joint mobility in the diabetic foot: relationship to neuropathic ulceration. Diabet Med 1988, 5(4):333-337.

40. van Deursen RW, Sanchez MM, Ulbrecht JS, Cavanagh PR: The role of muscle spindles in ankle movement perception in human subjects with diabetic neuropathy. Exp Brain Res 1998, 120(1):1-8.

41. Courtemanche R, Teasdale N, Boucher P, Fleury M, Lajoie Y, Bard C: Gait problems in diabetic neuropathic patients. Arch Phys Med Rehabil 1996, 77(9):849-855.

42. Cavanagh PR, Sims DS, Jr., Sanders LJ: Body mass is a poor predictor of peak plantar pressure in diabetic men. Diabetes Care 1991, 14(8):750-755. 


\section{Appendix}

Training program 
The training program was designed based on a study by Chetlin et al. (2004) and multidisciplinary consensus. It lasted 24 weeks, with 3 training sessions per week. Once a week there was a plenary training, consisting of four sections: a warm-up, strength and balance training, gait training and adjusted games. Apart from this plenary training the participants were asked to continue the strength training twice a week at home during 30 minutes.

\section{Warm-up}

The warm-up was the same every week, lasted for approximately 10 minutes and consisted of walking laps in the training hall at an individual pace. During walking various assignments were given:

- Stand still and stretch as far as you can: arms as high as possible over your head while standing on your toes;

- Stand still and 'roll down' starting with your head, vertebra by vertebra;

- Swing your arms and spin around;

- Walk on your toes:

- Walk on your heels;

- Walk with long steps;

- Walk with wide steps;

- Tap your heels against your buttocks;

- Pull up your knees;

- $\quad$ Shake loose your arms and legs.

\section{Strength and balance training}

The strength and balance training was performed sitting on a chair (during most exercises) with a duration of 25 minutes. The exercises were performed with constant resistance during 4 weeks. The first week started out with 3 sets of 4 repetitions. This was increased by 2 repetitions per week until 3 sets of 10 repetitions were performed in the fourth week. After the fourth and eight week the exercise resistance was increased and the participants started with 3 sets of 4 repetitions again, followed by the same schedule. The schedule is schematically presented in table 1.

Table 1. training schedule

\begin{tabular}{lllll}
\hline Week & $1 / 5 / 9$ & $2 / 6 / 10$ & $3 / 7 / 11$ & $4 / 8 / 12$ \\
Number of series $x$ repetitions & $3 \times 4$ & $3 \times 6$ & $3 \times 8$ & $3 \times 10$ \\
\hline
\end{tabular}

The strength and balance training consisted of two components. During the first 12 weeks of the training program it was mainly aimed at the lower leg; the exercises were:

- $\quad$ Resisted plantar flexion. Participant standing up, if necessary supported by a chair. Heel raises with repeatedly standing on toes. 
- Coordination of the lower leg muscles. Participant folds and unfolds a towel with feet while sitting on a chair.

- Resisted foot eversion. Participant sits, secures elastic band with predetermined resistance around balls of feet. Feet are placed at hip width on the heels, participant turns the right foot repeatedly from neutral position to eversion and back.

- Balance and stability exercises. Participants in twos, one performs the exercise, the other assists and offers support if necessary. Participant is standing behind a chair, feet at hip width, closes the eyes and stays standing, if possible without support. In the course of the weeks the difficulty level is increased by standing on one leg and finally to standing on one leg with eyes closed.

- Balance and stability exercises. Participants in twos, one performs the exercise, the other assists and offers support if necessary. Participant is standing behind a chair, feet approximately at hip width, lifts feet off the floor and holds the knee in $90^{\circ}$ flexion, closes eyes, if possible without taking support.

- Resisted dorsiflexion and plantar flexion. Participant sits, secures elastic band with predetermined resistance around balls of feet. Participant places heels on the floor, ankle in approximately $90^{\circ}$, the right foot moves toward plantar flexion and back, the left foot is kept still.

- Resisted dorsiflexion and plantar flexion of the foot. Participants in twos, sitting across from each other. One secures elastic band with predetermined resistance around the forefoot, while the other secures both ends under the foot. The first participant moves the foot repeatedly towards dorsiflexion and back to a $90^{\circ}$ angle.

The second component took place during weeks 13 until 24 and was aimed at strengthening the entire leg; the exercises were:

- Strength training of the hip and knee extensors. Participant sitting with elastic band with predetermined resistance under the right foot, the two ends secured in his or her hands. Starting with the knee at the chest, the leg is repeatedly extended towards the floor and slowly back to the starting position.

- Strength training of the hip and knee extensors. Participant standing in front of a step bench or comparable bench. Participant steps with one foot on the bench followed by the other foot. Then both feet return to the starting position.

- Strength training of the hip and knee extensors. Participants squat starting from a standing position (move buttock backward to relieve the knees), as if they want to pick something up from the floor. Then back to a standing position.

- Strength training of the hip and knee extensors. Participants standing with chair approximately $1 \mathrm{~m}$ in front of them. Participants make lunges forward, alternating right and left leg. Both legs are flexed, the knee of the hind leg in an angle of approximately $90^{\circ}$, with the knee moving towards the floor.

- Strength training of the hip and knee extensors. Participants sitting. Starting from this position each participant moves towards a full standing position, without using the 
arms/hands, and back to a sitting position. To ensure that the participants are actually sitting down, they must place a cone from the left side of the chair to the right side, or vice versa.

- Strength training of the hip adductors. Participant sitting with soft ball between the knees, the hands are kept on the back. Participant squeezes the ball empty between the knees, and slowly lets it inflate again.

- Strength training of the hip abductors. Participants sitting with elastic band with predetermined resistance which is knotted close-fittingly around the knees. The feet are approximately at hip width on the floor. The knees are alternately pulled away from each other and slowly back towards each other.

- Strength training of the knee flexors. Participants sitting with ball under the heel. Both hands are kept on the back, the ball is pressed flat with the foot, then slowly allowed to inflate.

\section{Gait training}

A gait training was offered during thirty minutes in the form of an exercise track, resulting in coordination training on a functional level.

- Diagonal ramp: ramp is diagonal to the walking direction (imitates slopes).

- Balance beam: path of $6 \mathrm{~m}$ long, $15 \mathrm{~cm}$ broad, two bends at right angles. The feet must be placed entirely on the strip while walking over the path.

- Walking on various surfaces (soft mattress, deep-pile carpet, grass, sand, rubber mats, foam rubber).

- Climbing a ramp, turn and back down: 2-3 benches attached to the wall bars next to each other at an obtuse angle (supervised).

- Cones are placed around the ends of a bench: participant walks in between the bench and the cones (imitates small passage, e.g. around a coffee table).

- Picking up balls from a box on the ground, bring it to another box.

- Stepping over a bench.

- Stepping stones: participant walks the track with long paces.

- Slalom: 6 cones with a stick standing in them, walk between them without touching the sticks or cones.

- Pirouette: on a disk with a diameter of $40 \mathrm{~cm}$, turn 360 degrees without moving off the surface of the disk.

- Sitting down and standing up without arm support, chair is $35 \mathrm{~cm}$ high. To ensure that the participants are actually sitting down, they must place a cone from the left side of the chair to the right side while sitting down, and vice versa.

- Walking while pushing a ball continuously with the foot, without losing control of the ball. 


\section{Games}

Each plenary training session was concluded with fifteen minutes of interactive games, such as volleyball, badminton or basketball. Because of the decreased load tolerance of the participants the games were adjusted, e.g. by replacing the ball with a balloon or beach ball. 
Nederlandse samenvatting

(Dutch Summary) 


\section{Inleiding}

Diabetes mellitus gaat een steeds belangrijkere rol spelen in onze gezondheidszorg. Het aantal patiënten met diabetes zal naar verwachting wereldwijd toenemen van 285 miljoen in 2010 tot 439 miljoen in 2030. De ziekte kenmerkt zich door een te hoog bloedglucose gehalte dat verschillende complicaties tot gevolg kan hebben. Diabetische polyneuropathie (DPN) is een van die complicaties en komt bij ongeveer $40 \%$ van de diabetes patiënten voor. Door het hoge bloedglucose gehalte kan er beschadiging optreden van de zenuwen in de armen en benen, met mogelijk pijn, verminderd gevoel en verlies van spierkracht als gevolg. Ook wordt DPN gekenmerkt door een verhoogd risico op moeilijk te genezen voetwonden. Verschillende factoren dragen bij aan dit verhoogde risico. Onze voeten worden door het lopen dagelijks belast, maar door het verminderde gevoel kan de patiënt met DPN minder goed waarnemen wanneer er overbelasting van de voeten plaatsvindt. Voetdruk kan als maat dienen voor deze (over)belasting en beschrijft de druk die inwerkt op de voetzool. Door DPN kan deze voetdruk toenemen en hoe hoger de voetdruk, hoe groter het risico op voetwonden. Het is echter momenteel niet volledig duidelijk waarom de voetdruk bij DPN patiënten verhoogd kan zijn. De huidige literatuur suggereert dat een verlies van spierkracht een rol zou kunnen spelen, maar hoe dit verlies van spierkracht kan leiden tot verhoogde voetdruk bij patiënten met DPN is nog niet volledig duidelijk. Het doel van deze dissertatie is dan ook om meer inzicht te verkrijgen in de onderliggende mechanismen die verantwoordelijk zijn voor het ontstaan van deze verhoogde voetdrukken. Daarnaast is onderzocht of training van de onderbeenspieren van patiënten met DPN een gunstig effect kan hebben op het looppatroon en de drukbelasting van de voet.

Om meer inzicht te krijgen in de beschikbare kennis die in andere onderzoeken is opgedaan, is een literatuuronderzoek uitgevoerd naar de aan het looppatroon gerelateerde factoren die voetdruk beïnvloeden (hoofdstuk 2). Daarnaast is een studie verricht met als doel de variabelen te optimaliseren die de voetdruk van DPN patiënten karakteriseren (hoofdstuk 3). Onderliggende mechanismen van verhoogde voetdrukken worden beschreven in de volgende hoofdstukken. Als eerste is onderzocht of een verminderde controle over de afwikkeling van de voet kan leiden tot verhoogde druk onder de voorvoet (hoofdstuk 4). Omdat deze verminderde controle over de afwikkeling van de voet veroorzaakt kan worden door een verlies van spierkracht ten gevolge van DPN, is vervolgens ook onderzocht of krachttraining van de beenspieren een gunstig effect kan hebben op de voetdruk (hoofdstuk 5) en het looppatroon (hoofdstuk 6).

\section{De invloed van het looppatroon op voetdruk bij mensen met DPN}

Hoofdstuk 2 beschrijft een literatuuronderzoek naar de invloed van het looppatroon op een verhoogde voetdruk bij patiënten met DPN. Uit de literatuur blijkt dat activatie van de onderbeenspieren een belangrijke rol kan spelen in het ontstaan van verhoogde drukken. Een beperkte controle over het krachtenspel in het onderbeen lijkt ook een belangrijke rol te spelen, maar het is nog onduidelijk hoe deze beperkte controle kan leiden tot verhoogde drukken. DPN kan ook leiden tot veranderingen in de karakteristieken van het looppatroon, zoals een verminderde loopsnelheid en kleinere stappen. Omdat deze veranderingen de voetdrukken beïnvloeden is het 
belangrijk om deze karakteristieken te meten en daar waar mogelijk te standaardiseren. Er zijn ook duidelijke aanwijzingen dat de belasting van de voet verminderd kan worden door het looppatroon aan te passen. Langzamer lopen, of het nemen van kleinere stappen kan zorgen voor een lagere belasting van de voet per stap. Daarnaast kunnen ook loophulpmiddelen worden ingezet om de fysieke activiteit van patiënten met DPN te verhogen, zonder dat dit leidt tot een toename van het risico op overbelasting van de voeten. Ook is het belangrijk om de fysieke activiteit zo veel mogelijk over de dag te verdelen. Vermoeidheid kan namelijk leiden tot veranderingen in het looppatroon met hogere drukken tot gevolg. Samenvattend laat het literatuuronderzoek zien dat het looppatroon een belangrijke factor is het ontstaan van verhoogde voetdrukken. Het is daarom belangrijk dat zorgprofessionals ook kijken naar eventuele mogelijkheden om het looppatroon aan te passen om zo een verhoogde druk te voorkomen.

\section{De druk-tijd-integraal als additionele maat voor de belasting van de voet}

Piekdruk is de meest gebruikelijke variabele om de voetbelasting te karakteriseren. Een andere variabele is de druk-tijd-integraal (DTI). Deze laatstgenoemde variabele is een maat voor alle druk die uitgeoefend wordt op een specifiek gebied gedurende een afgebakende tijd. Voor het bepalen van de belasting van de voetzool en het risico op voetwonden kan deze variabele waardevol zijn. Er is echter literatuur die aangeeft dat de toegevoegde waarde van de DTI ten opzichte van de piekdruk beperkt is. Echter, de software die meestal gebruikt wordt om de DTI te berekenen (Novel software) maakt alleen gebruik van de piekdruk over een bepaalde tijd en niet alle druk. In hoofdstuk 3 wordt daarom een nieuwe alternatieve berekening van de DTI geïntroduceerd. Aangezien druk gelijk staat aan kracht gedeeld door het oppervlak, is de DTI van een gebied van de voetzool berekend door de som van alle krachten in dat gebied te delen door de grootte van het contactoppervlak van dat gebied. Evaluatie van beide rekenmethoden van de DTI laat zien dat de nieuwe methode tot andere resultaten leidt dan de eerder gebruikte methode. Bovendien blijken deze verschillen groter te zijn in bepaalde gebieden van de voetzool en in bepaalde patiëntengroepen. Bij mensen met DPN werden de grootste verschillen waargenomen in de gebieden die een verhoogd risico hebben op het ontstaan van voetwonden. De nieuwe methode bevat waardevolle informatie en kan naast de piekdruk gebruikt worden als maat voor de belasting van de voetzool en daarmee als maat voor het risico op voetwonden.

\section{Onderliggende mechanismen van verhoogde voetdrukken}

Om meer inzicht te krijgen in de mechanismen die verantwoordelijk zouden kunnen zijn voor het ontstaan van verhoogde voetdruk, is een analyse uitgevoerd van het looppatroon van patiënten met DPN. In hoofdstuk 4 wordt beschreven dat het krachtenspel in de enkel samenhangt met het versneld naar voren verplaatsen van het drukcentrum (het punt van gemiddelde optredende druk) en dat daarmee ook een hogere belasting van de voorvoet plaats vindt. Spierzwakte ten gevolge van DPN zou dus kunnen zorgen voor een verminderde controle van de afwikkeling van de voet waardoor er een verhoogde belasting van voorvoet plaats kan vinden. Dit zou kunnen betekenen 
dat interventies die resulteren in meer spierkracht van de benen kunnen leiden tot een verlaging van de druk onder de voorvoet.

\section{De effecten van krachttraining op voetdrukken en het looppatroon bij mensen met DPN}

Hoofdstuk 5 beschrijft een interventieonderzoek naar de effecten van een door fysiotherapeuten begeleid krachttrainingsprogramma op de voetdrukken van mensen met DPN. Een groep patiënten met DPN kreeg, gedurende 24 weken, één maal per week een training aangeboden die bestond uit krachttraining, looptraining en sportactiviteiten. Deze patiënten werden gevraagd de krachttraining 2 maal per week thuis te herhalen. Een controle groep met vergelijkbare patiënten kreeg geen interventie. De voetdrukken werden gemeten op 0, 12, 24 en 52 weken na aanvang van het programma, tijdens een eigen gekozen loopsnelheid en een opgelegde (standaard) loopsnelheid. Uit de analyses bleek dat de interventie geen effect had op de voetdrukken over een periode van 52 weken. Wel bleek de piekdruk en DTI over deze periode toe te nemen met ongeveer $5 \%$, in zowel de interventie- als de controlegroep. Deze stijging in voetdruk duidt op een progressieve toename in de tijd van het risico op voetwonden bij patiënten met DPN. Het is momenteel nog niet duidelijk welke processen verantwoordelijk zijn voor deze toename. Het is daarom belangrijk dat er meer onderzoek plaats vindt naar de oorsprong van deze verhoogde voetdrukken en eventuele interventies om verhoogde voetdrukken te voorkomen.

Hoofdstuk 6 beschrijft de effecten van het eerder beschreven krachttrainingsprogramma op het looppatroon en het krachtenspel rondom de gewrichten van mensen met DPN. Ook deze metingen werden verricht tijdens de eigen gekozen en een opgelegde (standaard) loopsnelheid. Het trainingsprogramma had geen effect op de het krachtenspel rondom de gewrichten, maar zorgde wel voor een toename van lengte en de duur van een stap tijdens de opgelegde loopsnelheid. Waarom deze toename heeft plaatsgevonden is niet bekend, maar het is mogelijk dat dit het gevolg is van een betere controle over het looppatroon. Bovendien nam de eigen gekozen loopsnelheid toe over een periode van 52 weken in zowel de interventie als de controlegroep. Het lijkt aannemelijk dat deze toename in loopsnelheid het gevolg is van een leereffect dat veroorzaakt wordt doordat de patiënten steeds bekender worden met de testopstelling.

\section{Conclusies}

In deze dissertatie is aangetoond dat de DTI, mits berekend volgens de nieuw geïntroduceerde methode, informatie bevat die waardevol kan zijn voor de interpretatie van de belasting van de voet en dat deze belasting beïnvloed wordt door hoe iemand loopt. Ook is aangetoond dat het looppatroon een grote invloed heeft op de druk onder de voet. Bovendien kwam naar voren dat veranderingen in het looppatroon ten gevolge van DPN tot hogere voetdrukken kunnen leiden. Tijdelijke interventies die succesvol kunnen zijn in het voorkomen van hoge drukken, zijn onder andere het gebruik van loophulpmiddelen of het tijdelijk aanpassen van het looppatroon, zoals het nemen van kleinere passen en niet te snel lopen. Mogelijk kan ook de voetdruk onder de voorvoet worden verminderd door middel van krachttraining. Verminderde spierkracht zou er namelijk voor 
kunnen zorgen dat er een verminderde controle is van de afwikkeling van de voet, waardoor de voorvoet eerder belast wordt en de druk onder de voorvoet toeneemt. Het krachttrainingsprogramma beschreven in deze dissertatie had geen invloed op het krachtenspel rondom de enkel en de druk onder de voet, maar dit wil niet zeggen dat mensen met DPN geen baat hebben bij een krachttraining. Er zijn namelijk meerdere factoren die bepalen of een dergelijk programma succesvol is, zoals de intensiteit van de krachttraining of de motivatie van de deelnemers. Het is dus belangrijk dat de kennis uit deze dissertatie wordt gebruikt om nieuwe interventies te ontwikkelen om de belasting van de voet te verminderen. 
Appendix 
Dankwoord

(Acknowledgements) 
Dit proefschrift was nooit tot stand gekomen zonder de betrokkenheid van een grote groep mensen en organisaties. Via deze weg wil ik iedereen bedanken voor hun bijdrage.

Allereerst wil ik Nicolaas bedanken voor zijn geweldige sturing. De vele uren die we aan overleg besteed hebben waren zeer waardevol. Bedankt voor al die snelle en scherpe analyses, de kritische blik en de bijna niet stoppen vloedgolf aan ideeën.

Hans, je hebt een zeer grote rol gespeeld in dit onderzoek en de daaruit voortkomende artikelen. Ondanks je steeds drukkere agenda heb je altijd tijd vrijgemaakt voor het meedenken, analyseren en het lezen van stukken. Ik waardeer je inhoudelijke expertise, je optimisme, je nauwkeurigheid en vooral ook je openheid.

Ook wil ik Ton in het bijzonder bedanken voor al zijn hulp. Je uitvoerige meedenken en analyseren, soms zelfs met fictieve experimenten tot gevolg, heeft mij erg geholpen. Bovendien heb ik erg veel respect voor wie je bent en hoe je in het leven staat.

Kenneth, bedankt voor het meedenken, meelezen en de vooral ook je gezelligheid.

Ook wil ik graag iedereen bedanken die het proefschrift kritisch heeft gelezen en betrokken is geweest bij de beoordeling ervan.

Bovendien ben ik de deelnemers erg dankbaar voor hun inzet en bijdrage waardoor dit onderzoek plaats heeft kunnen vinden.

Natuurlijk wil ik ook Herman bedanken. Het was een geweldige kans om samen te werken met een vriend. Ook door jouw bijdrage, je gezelligheid en vooral ook je humor kijk ik terug op een mooie periode.

Paul, je ondersteuning bij de experimenten en analyses was echt onmisbaar. Als er een oplossing bestaat, dan weet jij hem te vinden.

Patty, je was een waardevolle bijdrage voor het team. Tijdens je stage bleek al hoe nauwkeurig en ijverig je bent. In de lange periode die je ons daarna nog hebt geholpen, heb je dat beeld nog verder bevestigd.

Elise, Ruud, Janneke, Rebecca, Tijn en Rens, bedankt voor alle hulp bij de uitvoering van de trainingen. Jullie hebben laten zien hoe leuk bewegen kan zijn.

Valeria, bedankt voor je hulp en adviezen. Je hebt ons de statistische skills en tools gegeven om onze data correct te analyseren.

In het Maastricht Universitair Medisch Centrum + wil ik graag de afdeling interne geneeskunde, neurologie, fysiotherapie en podotherapie bedanken en in het bijzonder Ton, George en Jean voor 
een prettige samenwerking, hulp bij rekrutering en het beschikbaar stellen van ruimtes en ondersteuning.

Ook de verschillende internisten, podotherapeuten, en verpleegkundigen uit Máxima Medisch Centrum die hebben bijgedragen aan dit onderzoek wil ik graag bedanken en in het bijzonder Louis, Hanny, Ingrid en Annelies voor het meedenken en jullie actieve rol in de rekrutering en uitvoering van het onderzoek in Eindhoven.

Daarnaast wil ik ook graag de afdeling interne geneeskunde van het St. Anna Ziekenhuis in Geldrop en in het bijzonder Arno bedanken voor de hulp bij de rekrutering.

Ook wil graag alle collega's van Fontys Paramedisch Hogeschool en in het bijzonder de opleiding Fysiotherapie en mijn kamergenoten bedanken voor een erg prettige samenwerking in het onderwijs, maar natuurlijk ook voor alle gezelligheid! Eveline en Benedicte, bedankt voor jullie adviezen. Frank en Rienk, bedankt voor alle vrijheid die jullie me hebben gegeven. Tim en Patrick, bedankt voor jullie meedenken maar vooral ook jullie gezelligheid en humor. Paul, bedankt voor je enthousiasme en vertrouwen.

Esther, bedankt voor je creativiteit!

Désirée, je ondersteuning bij het opzetten van het onderzoek in Eindhoven was zeer waardevol.

Miranda, Karin en Johan, bedankt voor jullie flexibiliteit en dat ik op jullie grondgebied ons meetlaboratorium mocht realiseren.

Theresa, bedankt voor het creëren van mogelijkheden en je interesse.

Antal, Ralph en Jan-Willem, bedankt dat jullie altijd stand-by stonden voor ondersteuning bij de bloedafnames.

Hanny, bedankt voor je engelengeduld en het regelen van de vele afspraken in Maastricht.

Natuurlijk wil ik ook mijn collega's van Universiteit Maastricht bedanken. Janneke, Rachel, Marieke en alle anderen bedankt voor jullie gezelligheid en het delen van jullie ervaringen.

Alle vrienden en kennissen wil ik bedanken voor hun interesse, de ontspanning en de lol die jullie me hebben gegeven. Diane, Ralf en Mathieu, jullie wil ik naast de gezelligheid ook bedanken voor alle adviezen en het delen van jullie eigen ervaringen.

Daarnaast wil ik natuurlijk ook mijn familie en schoonfamilie bedanken voor hun betrokkenheid en interesse. Mijn vader en moeder wil ik daarbij in het bijzonder bedanken. Jullie hebben me altijd gestimuleerd in het leren en het studeren. Daarin hebben jullie me in alle mogelijke manieren ondersteund. Ik ben dan ook trots dat jullie mijn ouders zijn! 
Tot slot, lieve Laura, je bent een hele grote steun voor me. Je begrijpt me! Je geeft me het vertrouwen en de energie die ik nodig heb! Dank je wel! 
Curriculum Vitae \& Publications 


\section{Curriculum Vitae}

Tom Melai was born in Roosendaal en Nispen, on the $3^{\text {rd }}$ of April 1981. He attended secondary school at the Thomas More College, after which he moved to Maastricht to study General Health Sciences, with a specialization in Human Movement Sciences, at Maastricht University. In 2003 he also attended Zuyd University of Applied Sciences in Heerlen to study Physical Therapy and he obtained both his degrees in Physical Therapy as well as General Health Sciences in 2006.

During and after his education Tom worked as tutor at the Faculty of General Health Sciences at Maastricht University. At the end of 2006 he obtained a position as lecturer and PhDstudent at Fontys University of Applied Sciences at the Institute of Allied Health Professions in Eindhoven. As a lecturer he was closely involved in the coordination and development of education on biomechanics, applied sciences and the graduation project for physical therapists. As the first PhD-student at the Institute of Allied Health Professions he set-up and performed the clinical trial that led to the current dissertation.

\section{Publications in peer reviewed journals}

IJzerman H, Schaper N, Melai T, Lima Passos V, Meijer K, Willems P, Savelberg H. Resistance training improves plantar flexor strength and walking speed in people with diabetic polyneuropathy. Submitted.

Melai T, Schaper N, De Lange A, Savelberg, H. The influence of gait in diabetic polyneuropathy on plantar pressures: a review. Submitted.

Melai T, Schaper N, IJzerman H, Willems P, De Lange A, Meijer K, Lieverse A, Savelberg, H. Strength training affects lower extremity gait kinematics, not kinetics, in people with diabetic polyneuropathy. A randomized controlled trial. Journal of Applied Biomechanics, 2013. Accepted for publication.

Melai T, Schaper N, IJzerman H, De Lange A, Willems P, Lima Passos V, Meijer K, Lieverse A, Savelberg, H. Lower leg muscle strengthening does not redistribute plantar load in diabetic polyneuropathy. A randomized controlled trial. Journal of Foot an Ankle Research, 2013. Provisionally accepted for publication.

Melai T, IJzerman H, Schaper N, De Lange A, Willems P, Meijer K, Lieverse A, Savelberg, H. Increased forefoot loading is associated with an increased plantar flexion moment. Human Movement Science, 2013. In press.

IJzerman H, Schaper N, Melai T, Meijer K, Willems P, Savelberg H. Lower extremity muscle strength is reduced in type 2 diabetic patients, with and without polyneuropathy, and is associated with impaired mobility and reduced quality of life. Diabetes Research and Clinical Practice. 2012; 95(3): 345-51. 
IJzerman H, Schaper N, Melai T, Blijham P, Meijer K, Willems P, Savelberg H. Motor nerve decline does not underlie muscle weakness in type 2 diabetic neuropathy. Muscle \& Nerve. 2011; 44(2): 241-5.

Melai T, IJzerman H, Schaper N, De Lange A, Willems P, Meijer K, Lieverse A, Savelberg H. Calculation of plantar pressure-time integral, an alternative approach. Gait \& Posture. 2011; 34(3): 379-83.

\section{Other publications/ conference proceedings}

Melai T, IJzerman H, De Lange A, Schaper N, Willems P, Meijer K, Savelberg H. Adverse plantar pressures are associated with increased plantar flexion moments at the first part of the stance phase. Nederlands Tijdschrift voor Diabetologie. 2011; 11(9): 127.

IJzerman H, Schaper N, Melai T, Willems P, Meijer K, Savelberg H. Specific strength training enhances muscle strength and functional mobility in diabteic patients with polyneuropathy. Nederlands Tijdschrift voor Diabetologie. 2011; 11(9): 127.

Melai T, IJzerman H, De Lange A, Schaper N, Willems P, Meijer K, Savelberg H. Assessing plantar loading in neuropathic gait. Proceedings of the Diabetic Foot. Noordwijkerhout, May 2011.

Melai T, IJzerman H, De Lange A, Willems P, Meijer K, Schaper N, Lieverse A, Savelberg H. Calculation of plantar pressure-time integral, an alternative approach. Proceedings of the ESM. Rhode Island, August 2010.

IJzerman H, Melai T, Meijer K, Schaper N, Willems P, Savelberg H. Relation between muscle strength and mobility in diabetic patients with and without diabetic polyneuropathy. Nederlands Tijdschrift voor Diabetologie. 2009; 11(7): 123-4.

Savelberg H, IJzerman H, Melai T, Schaper N, Willems P, De Lange A, Meijer K. Changes in the ankle joint moments underlie redistribution of plantar pressure patterns. Nederlands Tijdschrift voor Diabetologie. 2009; 11(7): 119-20.

Melai T, De Lange A. Foot loading during different walking speeds: is there a balance between peak pressure and pressure-time integrals? Proceedings of the IVO. Den Haag, November 2009.

Melai T, IJzerman H, De Lange A, Willems P, Meijer K, Schaper N, Savelberg H. Increased forefoot loading in diabetic polyneuropathy is linked to diminished braking force after heel strike. Proceedings of the IVO. Den Haag, November 2009. 
Melai T, IJzerman H, De Lange A, Willems P, Meijer K, Schaper N, Savelberg H. Gait analysis in diabetic polyneuropathy. Gait and Posture. 2008; 28(s2): S87-S88.

Melai T, Senden R, IJzerman H, Savelberg H, Meijer K. Qualification and characterization of tripping incidents using accelerometry. Proceedings of the 4th International Biomechanics of the Lower Limb in Health Disease \& Rehabilitation. Salfort, September 2007. 


\section{Propositions}

\section{Belonging to the dissertation entitled}

\section{Plantar pressures in diabetic polyneuropathy: the influence of gait and exercise therapy}

1. The pressure time integral provides clinically relevant information on the risk of plantar tissue damage. (this dissertation)

2. Higher forefoot loading can be the result of an impaired muscle function of the dorsal and plantar flexors of the foot. (this dissertation)

3. Plantar pressures in people with diabetic polyneuropathy can be lowered by changing the gait pattern. (this dissertation)

4. Lower leg muscle training for people with diabetic polyneuropathy needs to be tailor-made to avoid a high drop-out. (this dissertation)

5. As plantar pressures can increase rapidly over time, plantar pressures should be monitored regularly in every patient with diabetic polyneuropathy to identify and evaluate plantar areas at risk for ulceration. (this dissertation)

6. Monitoring or standardizing gait velocity is essential to monitor plantar pressures correctly.

7. Evidence based practise should be part of every allied health profession, but it is not necessary for every allied health professional to be a researcher.

8. For most chronic diseases, physical activity is the best medicine there is.

9. Standing still will lead to (tissue) degeneration.

10. If you make a well-informed decision, you should never regret it. 


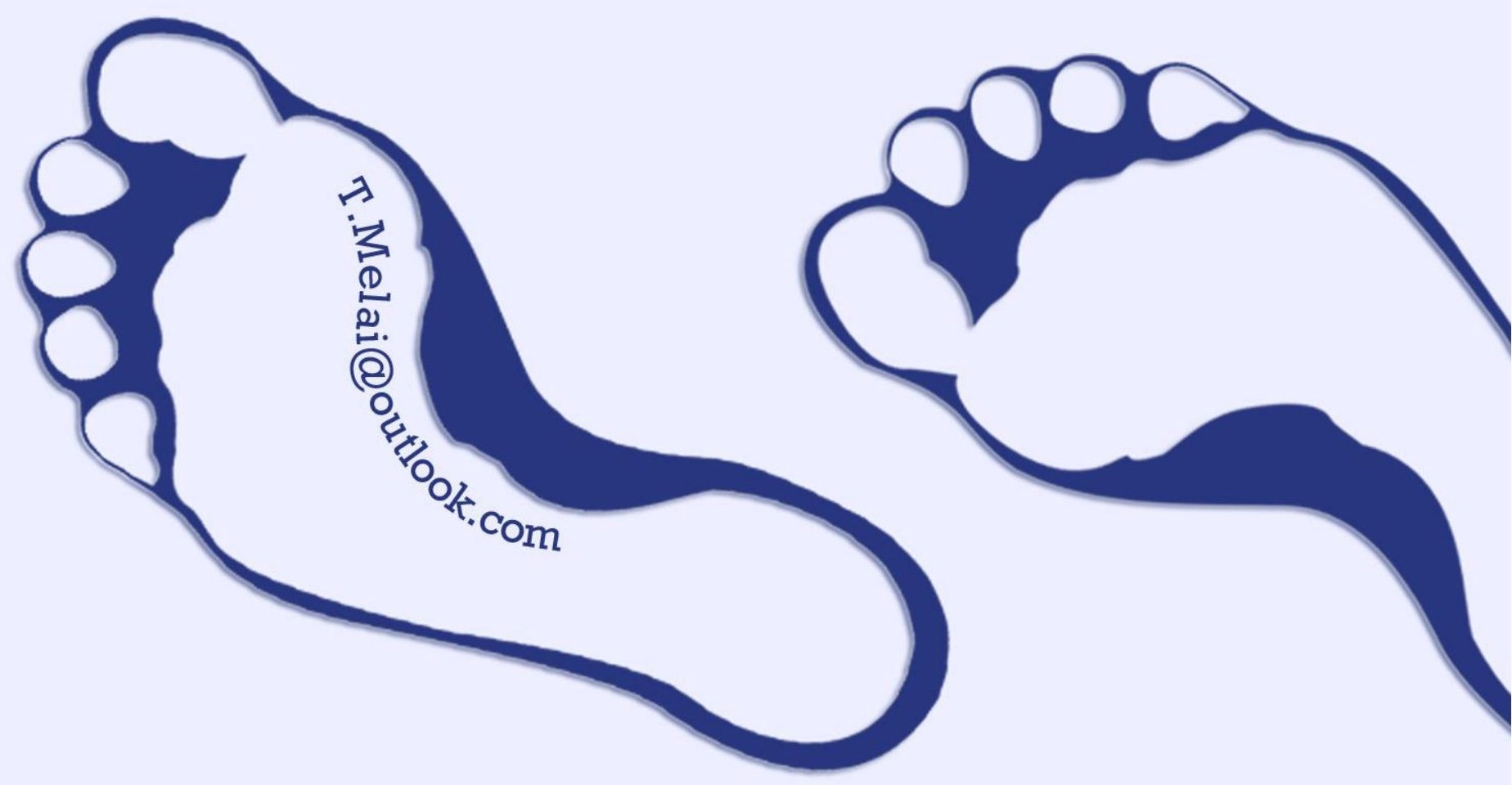

